\title{
Determinants of water and ion permeation through nanopores studied by Molecular Dynamics simulations.
}

\author{
Dissertation \\ zur Erlangung des Doktorgrades \\ der mathematisch-naturwissenschaftlichen Fakultäten \\ der Georg-August-Universität zu Göttingen
}

Vorgelegt von

Guillem Portella Carbó

aus Girona, Spanien

Göttingen, 2008 
D 7

Referent: Prof. Dr. Ulf Diederichsen

Koreferent: Prof. Dr. Helmut Grubmüller

Tag der mündlichen Prüfung: 30.04.2008 




\section{Abstract}

Membrane channels facilitate the transport of matter through the cell membrane, and therefore play a crucial role in life. The knowledge of the structurepermeability relationships in model channels is essential for the understanding of natural membrane channels and for the design of novel channels with desired characteristics. The aim of this thesis is towards the characterization of the critical fundamental factors that determine water and ion permeation through channels of molecular dimensions. To this end, we performed atomistic molecular dynamics simulations to extract crucial energetic and dynamic properties underlying water and ion permeation in nanopores with different geometries and polarities. First, we show that a systematic approach where only one property is varied at the time is required. We then use a series of designed peptidic channels that permeate water in single-file regime to isolate the influence of the channel length on the water mobility. The extension of the channel is found to have no impact on the water mobility. In contrast, we show that the polarity of the channels strongly influences the water permeability, ranging from almost empty pores to tightly adsorbed water molecules. The channel polarity corresponding to the natural peptidic channels is found to be close to optimal for water permeation. The water mobility within a given water-pore affinity remains invariant with the length. Moreover, by systematically varying the radius and the polarity of model pores, we find a strong effect of the pore radius and polarity on the water pore occupancy and water permeability over a range of radii of $0.4 \mathrm{~nm}$. Water permeabilities span two orders of magnitude as the pore radius increases, approaching the macroscopic radial dependence at large radii. Finally, we show that free energy barriers for ion permeation through single-file pores display a strong length dependence. A central barrier emerges until saturation as the channels elongates, correlating with desolvation of the ion from the bulk. Whereas the main contribution to the ion permeation free energy barrier is found to be 
entropic, the length dependence of the permeation barrier is dictated by the enthalpic contribution. Implications of the presented results for the understanding of natural channels and for the design of novel channels are discussed throughout this thesis. 


\section{Acknowledgments}

I would like to start this thesis by sincerely thanking everyone that, directly or indirectly, contributed to this work.

First, I would like to thank Dr. Bert de Groot for the opportunity to join his research group and for motivating me to work on the topics presented in this thesis. Bert has always been there, coping with my doubts and mistakes with extreme patience, offering practical solutions for problems that seemed insurmountable.

I would also like to thank Prof. Helmut Grubmüller, director of the department of Theoretical and Computational Biophysics at the MPI-BPC, for providing the members of the department with an exceptional working environment. Thanks to Eveline Heinemann for the great work at the secretary. Thanks to Ira Tremmel and Katja Riebeseel for proof reading manuscripts for publication, one of them being this thesis. Thanks to Ingo Hoffman, Ansgar Esztermann and Martin Fechner for the professional computer administration.

Thanks to all the present and past members of the Theoretical and Computation Biophysics department for their support, interesting scientific discussions and good times. I learned a lot from everyone here. Special mentions: Camilo Aponte, Benjamin Bouvier, Frauker Gräter, Gerrit Groenhoff, Jürgen Haas, Carsten Kutzner, Oliver Lange, Maik Götte, Lars Schäfer, Daniel Seeliger, Esteban Vöhringer-Martinez and Ulrich Zachariae. I would specially like to thank Nicole Dölker for her help with german translations, and Martin Stumpe for organizing the groups seminars. Thanks to Marcus Kubitzki and Jochen Hub for their patience with my questions and practical problems. Finally a note of gratitude to Harshad Joshi for his friendship, for providing a great office environment and sharing his Gromacs expertise.

On my personal life in Göttingen, thanks to my flatmates Susanne and Mitja, specially in the last months, for providing a comfortable and enjoyable atmo- 
sphere at what developed into my home. Thanks to Itzam de Gortari, Rodrigo Martinez, Wilson Quevedo, and Laura Riolobos for all the fun, moral support when needed, and for making me feel as if I had a real life once in a while. It was a relative short period of time, but since I tend to remember only the good things, they will surely be part of my memories.

Back at home, my real home at Girona, thanks for the support and good vibs from my good friends, Jordi, Pere and Ernest. They will never read this thesis, and they will never care about its contents, thanks also for that. Núria, for her love, support, patience, commitment and shared hopes. For enduring the long wait.

Thanks to all the members of my family, aunts and cousins, who actively supported me during my stay in Göttingen. I know they will always be there for me. Finally the most special thanks to my brother Ferran, my mother Carme and my father Jaume. Thanks to them for their steady support, courage and love. My parents are my guiding torch, the architects of my education.

Göttingen, 19 of March 2008 


\section{Contents}

$\begin{array}{lr}\text { 1. Introduction } & 1\end{array}$

2. Theory and Methods $\quad 11$

2.1. Molecular Dynamics principles . . . . . . . . . . . . . 11

2.2. Derivation of permeation coefficients . . . . . . . . . 17

2.2.1. Definition of the permeability coefficients . . . . . . . . 17

2.2.2. Permeability coefficients from equilibrium simulations . . . 19

2.3. Free energies and molecular dynamics simulations . . . . . . . . 23

2.3.1. Methods to extract free energies from simulations . . . . . 25

3. Gramicidin channels: a starting point 33

3.1. Gramicidin and midigramicidin ... . . . . . . . . . 33

3.2. Results and discussion . . . . . . . . . . . . . . . 36

3.3. Conclusions and outlook . . . . . . . . . . . . . . . . . 42

4. Influence of the pore length and polarity on water permeability 45

4.1. Length dependence of water permeability . . . . . . . . . . . . 46

4.1.1. Introduction . . . . . . . . . . . . . . . 46

4.1.2. Theory and Methods . . . . . . . . . . . . . . . . . 47

4.1.3. Results and discussion . . . . . . . . . . . . . 53

4.1.4. Summary and Conclusions . . . . . . . . . . . . 59

4.2. Pore polarity effects on water permeability length dependence . . 61

4.2.1. Results and discussion . . . . . . . . . . . . 63

4.2.2. Summary and Conclusions . . . . . . . . . . . 70

5. Influence of the pore radius and polarity on the water permeability $\mathbf{7 3}$

5.1. Introduction and previous studies . . . . . . . . . . . 74

5.2. Theory and Methods . . . . . . . . . . . . . . 75 
5.3. Pore water occupancies and density as function of radius and pore polarity ......................... 78

5.4. Permeability coefficients as function of the radius . . . . . . . . 84

5.5. The breakdown of proportionality between $\langle\mathrm{n}\rangle$ and $\mathrm{p}_{\mathrm{f}} / \mathrm{p}_{\mathrm{d}} \ldots \ldots 8$

5.6. Summary and conclusions . . . . . . . . . . . . . . . . 94

6. Entropy reduction increases single-file ion permeation barriers $\quad 97$

6.1. Introduction . . . . . . . . . . . . . . . . . . . . . . 98

6.2. Theory and Methods . . . . . . . . . . . . . . . . 99

6.3. Results and discussion . . . . . . . . . . . . . . 106

6.3.1. Polyalanine channels . . . . . . . . . . . . . . . . 106

6.3.2. Ring structured channels . . . . . . . . . . . . . . . 110

6.3.3. Origin of system's entropy reduction . . . . . . . . . . . 112

6.4. Summary and conclusions . . . . . . . . . . . . . . . 117

$\begin{array}{ll}\text { 7. Summary and Conclusions } & 119\end{array}$

$\begin{array}{ll}\text { A. Appendix } & 125\end{array}$ 


\section{Introduction}

The spatial confinement in cells by a lipidic plasma membrane is a prerequisite for life. It guarantees, e. g., an efficient control over the chemical and physical processes from which organisms obtain the necessary driving energy to function. Cells must be, however, able to exchange energy and matter with their environment. Depending on their composition ${ }^{1}$, lipid membranes are permeable via simple passive diffusion to small uncharged nonpolar molecules, such as oxygen and carbon dioxide, to a lesser extend also to water molecules, urea and glycerol, and almost impermeable to ions. At a larger scale, endo- and exocytosis are mechanism to introduce or excrete huge amounts of matter to/from cells and cellular compartments by means of membrane fission/fusion.

Other fundamental characteristics of living organisms are the response to external stimuli and the auto-regulation to maintain a constant operative state. Adequate reaction to changes in the environment, be it a change of $\mathrm{pH}$, a change in temperature or light intensity, requires precise physiological machinery. The response to such stimuli is done by a simple mechanical transduction or more complex biochemical pathways. The propagation of signals and their corresponding response requires myriads of inorganic ions, water, sugars, and molecules of diverse size to be transported selectively, on demand and in very specific locations, including the passage of cellular and subcellular membranes. A simple diffusion mechanism through the membrane would be too slow and unselective to accomplish such a task. These considerations and the phenomena of osmosis $[2,3]$ led to the so-called pore hypothesis [4-6]. Decades of biophysical and biochemical essays finally resulted in the discovery and structural characterization of ion [7-9] and water channels [10, 11]. It is nowadays a well-established

\footnotetext{
${ }^{1}$ The lipid composition in the plasma membrane depends on the function of the cell and its location, e. g., the apical plasma membrane in cells located in the kidney collecting duct has a very low water permeability. Other membrane compositions, such as cytoplasmic bilayers, display a much larger permeability [1]
} 
fact that water and ion channels are proteins embedded in cell membranes [7, 10], and that they play essential roles in bioenergetics, neurobiolgy, and in a large number of pathophysiological conditions.

The proteins that facilitate the permeation and transport of matter through the cell membrane are categorized into two groups: pores or channels ${ }^{2}$, and carriers or transporters. Channels facilitate the passive diffusion of species through the membrane by providing a pathway of appropriate characteristics. Channels do not usually undergo large conformational changes to allow transport, although a large portion of them are gated, $i$. e., an external driving force can open or close the channel. Since no source of energy is used to bias the diffusion of the permeants, their directional motion is dictated by a concentration gradient between the two separate compartments. In the absence of driving forces, no net transport between the two compartments occurs. Carriers or transporters require a conformational change to allow the passage of solutes. They facilitate the transport of solutes down their concentration gradient, but they can also drive them against it. This last possibility requires an external energy source, which can be obtained from a biochemical reaction, from light harvesting or from the coupling to the spontaneous transport of another solute down their own concentration gradient (cotransporters).

The complex machinery that operates in cells requires highly specialized control mechanisms. The vast majority of the channels and carriers are therefore regulated, and can be activated (opened) or deactivated (closed) by physicochemical means. Examples of regulatory mechanisms are ligand binding to a particular site, from where the signal is amplified to activate/inactivate the function of the protein, plugging of the permeation pathway by a ligand, or the gating by a voltage differences across the membrane. Other mechanisms include pressure induced gating in mechanosensitive channels [12, 13], temperature gating $[14,15]$ and $\mathrm{pH}$ sensing [16]. Recently, the notion emerged that hydrophobic stretches along the channel pathway could aid the gating of ion channels [17, 18].

Water and ions, essential for life The most abundant component in cells is liquid water. Its special properties and ubiquity on our planet makes water the solvent of life [19]. One of the special characteristics of water is the network

\footnotetext{
${ }^{2}$ The terms pore and channel will both be used in this thesis to specify membrane-embedded channels that facilitate the passive permeation of solutes or water across the membranes.
} 
of hydrogen bonds, which explains its high boiling point temperature despite its low molar weight. The hydrogen bonding between water molecules, a direct consequence of its electronic structure, is responsible for most of the properties of water. Liquid water has multiple vital roles in cells: it acts as the solvent in which biomolecules and ions diffuse, it lubricates and stabilizes (macro)molecular structures [20, 21]. Due to its amphoteric nature, water molecules act as catalyst in multiple reactions. Photosynthesis depends on the photolysis of water molecules to generate protons, which are used to drive the synthesis of the energetic currency of cells.

Inorganic ions, such as sodium, potassium, calcium, iron, chloride and phosphate ions, are found in solution in the cytosol of cells, complexed in prosthetic groups of proteins and stabilizing the structure of proteins and nucleic acids [22]. They are used to generate electrostatic potentials that propagate the electrical impulses in neuron cells, mediate protein-protein and protein-DNA recognition, regulate the osmotic pressure in cells, catalyze chemical reactions, etc.

Water, and specially ions, require special pathways to cross the low dielectric medium of lipid membranes of cells and organelles. Since channels do not depend upon large conformational changes to facilitate the transport through the lipid membrane (once in the active state), the turn-over rate for permeation is typically very high ${ }^{3}$. Such fast permeation rates are needed to allow a rapid response to external stimuli. Besides the previously mentioned energetic and regulatory properties, naturally occurring pores are characterized by their specificity towards a subset of permeants. To achieve selectivity, nature has developed a variety of mechanisms to filter desired molecules (and even atoms) without compromising their efficiency. In the vast majority of cases, selectivity is accomplished by suitable pore size in conjunction with electrostatic barriers [2429]. Aquaporins are virtually impermeable to ions and protons [30]. Biologically relevant ions, such as sodium, potassium and chloride, are facilitated through membrane proteins which exhibit exquisite selectivity for a given species. Selective channels are universally endowed with narrow regions [7, 27], where the flow of matter is filtered based on the size and electrostatic signature of the permeant molecules. To this end, the transport through the selective region of ion and water channels usually occurs in a single-file fashion: the permeating species are

\footnotetext{
${ }^{3}$ The turn-over rate is in the order of nanoseconds for a water molecule permeating an Aquaporin 1, the water channels of the cell, in the absence of an imposed gradient [23].
} 
arranged in line, such that a direct interaction with the protein atoms in the filter region is established.

Water confined by channel geometry to a one-dimensional file of molecules interacting with the pore can exhibit a variety of distinct types of behavior. Depending on the radius and polarity of the channel, the thermodynamic state of the water molecules in the pore varies. In hydrophilic channels, water molecules are stabilized by electrostatic interactions, and therefore the pore interior is filled by water most of the time. At low polarity, however, the state of water molecules can be described by a liquid-vapor equilibrium [31-33]. Water molecules permeate such pores in clusters of water molecules tightly connected by hydrogen bonds, which can not find other partners on the channel pathway.

Experimental determination of ion and water permeation Quantitative measurements of water and ion permeation are crucial to elucidate the nature and the function of channels in the cell membrane. The evidence of an oily membrane surrounding cells and its permeability properties became clear after the study of osmotic effects on red blood cells and plant cells [34]. A combined effort between biologists, chemists and physicists developed in the understanding of the connection between diffusion, osmotic pressure and transport through oily membranes. Furthermore, the low permeability for ions and charged species led to the hypothesis of aqueous pathways in the cell membrane [7] .

The activity of ion channels is typically characterized by their electrical currentvoltage relationships and for their time dependent electrical current profiles. Whenever ions are permeating the channel, a corresponding current is measured as a function of time, which allows to quantify the open/closed states of the channel [35]. To perform single channel electrical recordings, the patch-clamp technique [36] is commonly used.

The most direct method to observe the permeation of water through semipermeable membranes consists in establishing a net flux that produces a measurable change in volume in the recipient compartment. A spontaneous net flux of water molecules through the membrane can be readily induced by a difference in the chemical potential between the two compartments separated by the membrane, $i$. e., via osmosis. The osmotic permeability coefficients $P_{f}$, relates the net flux of water through that membrane to the difference in concentration between the two 
compartments. In this sense, the osmotic permeability measures the response of the membrane to an external perturbation.

Several methods for measuring the osmotic permeability of membranes exist $[37,38]$. If permeable pores are embedded in the membrane, there is an increase in the amount of water that can cross the bilayer. This results from the sum of the intrinsic osmotic permeability of the membrane and the permeability of embedded channels. Channels can be reconstituted in model planar lipid membranes, in liposomes or expressed in oocytes. Widely used techniques involve the decay in the electric current due to dilution of one compartment caused by the water flux [39, 40], or measuring optical properties such as light scattering, or gravimetric techniques [38]. Single-channel permeability measurements are accomplished by monitoring the total permeability coefficient of the membrane, the permeability coefficient of the membrane in the presence of an increasing number of channels, and by quantification of the number of open channels.

Another permeability coefficient to characterize permeation through membranes, and especially channels, is the diffusive permeability coefficient $P_{d}$. The diffusive permeability is a measure of the equilibrium flux between compartments of the same osmolality [5]. Since no volume changes or electrolites dilution take place, the diffusive permeability uses isotopically labeled water molecules to follow the water flux $[41,42]$. Osmotic and diffusive permeability coefficients have different values depending on the presence or absence of pores in the membrane. If the membrane has no pores that facilitate the permeation of water molecules, the osmotic and diffusive permeability coefficients have the same value. A membrane with embedded pores that only allow water to permeate has an osmotic permeability coefficient larger than than the diffusive permeability [5]. Measuring both the osmotic and the diffusive permeabilities allows the detection of water pathways in the membrane.

Channels and (bio)technology Pores play a fundamental role in nature as well as in nano-technological applications. Water-selective pores, such as Aquaporins, are suitable as filtering devices [43]. Due to their small size an selectivity, ion channels are used as sensible detectors embedded in supported bilayers, with multiple applications [44-48]. Although the details vary depending on the application, a common principle is the modulation of the single-channel cur- 
rent recordings upon interaction with the analyte. The small antibiotic peptide gramicidin A, which will be introduced shortly and was also studied in this thesis, is known to selectively permeate monovalent cations along with water. The small diameter of this pore $(4 \AA)$ accounts for its selectivity, because even small molecules like urea cannot fit in its lumen. This selectivity makes gramicidin A channel very interesting as sensitive detector, and has been extensively used for this purpose. Example applications include the detection of protein-lingand interaction [49-51] and pH sensors [52]. Other popular choices for biology inspired pore-based sensing devices are engineered peptidic nanotubes [53-55] and $\alpha$-hemolysin [56].

\section{The role of computational studies in the understanding of channel per-} meation The interplay between experiments and theory are at the heart of the scientific method. The development of theories and models that describe the empirical evidences, and offer testable predictions, proved highly successful to advance the understanding of the physical world. The method of molecular dynamics simulations is a powerful approach to study both dynamic and thermodynamic properties of a system, e. $g$. the diffusion coefficient or the heat capacity of a liquid can be extracted by this method. Early rudimentary simulations of hard sphere liquids were already used by Levitt in 1973 [57] to study dynamics properties of single-file transport. Currently, methods with more realistic description of the interactions between ions, water molecules and channel atoms are widely used [29, 58-60]. Molecular dynamics simulations with atomistic resolution can offer detailed insights in the nature of water and ion permeation [31, 61-63]. This is particularly valuable for permeation of ions and water through nanoscopic pores, since currently no experimental method allows to visualize the behavior of ions and water as they permeate the channel in 'real time'. Furthermore, the influence of several contributions can be systematically studied in ways that are not accessible by experimental essays, providing input for further experimental work and testable hypotheses. One major drawback in molecular dynamics simulations is that the typical time scale computationally affordable can be several orders of magnitude lower than the experimental time resolution. 


\section{Aim of this thesis}

The aim of this thesis is towards the characterization of the critical fundamental factors that determine water and ion permeation through pores of molecular dimensions. The knowledge of the structure-permeability relationship in model peptidic channels, such as gramicidin A and its derivatives, is relevant for the understanding of natural channels. Furthermore, computational studies of atomistic resolution offer a detailed view of the relevant contributions for the design of novel channels with desired permeability and selectivity characteristics.

Due to the small diameter of selective ion channels, such as gramicidin A, the permeation of water molecules occurs in the single-file regime. If wider channels are considered, the single-file character of the transport might not be conserved, and even lost for pore radii larger than the diameter of a water molecule. To obtain permeability coefficients from our simulations, it is desirable to employ a methodology whose underlying assumptions are applicable to different permeation regimes. Since no theoretical expression for the osmotic or diffusive permeability proposed up to date accounts for all permeability regimes, a computational strategy is a valuable approach. Complementary to the dynamic characterization, extensive energetic analyses of the contributions to the permeation barrier for ions and water through such model pores provide valuable insights into the nature of the transport.

A gradual approach, motivated by experimental and computational observations, was followed to determine and characterize crucial determinants like the pore length, radius and polarity, underlying the permeation of water and ions through nanopores. Besides the Theory and methods overview in chapter 2, the present thesis covers the following topics:

Gramicidin A and its derivatives as model peptidic pores This study, presented in chapter 3, serves as a starting point for the rest of the thesis. Gramicidin A and cross-linked midigramicidin peptidic channels, embedded in a model lipid membrane of dimyristoylphosphatidylcholine, are studied to extract relevant factors underlying water permeation. Both channels conduct water in the single-file regime due to their narrow lumen. The effect of the inserted peptides on the lipid membrane, their average orientations with respect to the bilayer, and the role of the tryptophan residues of the peptide are discussed. From our simu- 
lations, the lipid head groups are clearly identified as deterrents of the water flux due to their ability to block the channel entrance. Based on this observation, we suggest modifications of the peptide's sequence to test the hypothesis that a reduction of the lipid head group interference increases the water permeability. The computed permeability coefficients indicate that midigramicidin permeates water at faster rates than gramicidin A, in agreement with experimental measurements. However, a clear structure-permeability relationship does not become apparent, as a number of differences are present between the two channels. Besides the length, also the radius and the arrangement of capping groups is different. In this respect, this work highlights the complex interplay between membrane distortion upon peptide insertion, the stability of the peptide's secondary structure, the channel geometry and the permeability coefficients. Consequently, a systematic approach where only one property is probed at a time is necessary. The rest of the thesis is concerned with such studies.

Reduction of complexity, one step at a time Following the results in the gramicidin A and midigramicidin systems in lipid bilayers, together with experimental findings indicating that osmotic permeability coefficients decay exponentially with the channel length [64], we designed a series of systematic studies of putative permeability determinants. In chapter 4 we explore the effects of the length on the permeability coefficients. To this end, we constructed a series of gramicidin-like channels of increasing length and fixed radius, that were embedded in an artificial octane membrane to avoid disturbances at the channel entrance. Our results indicate that water permeability is independent on the pore length, at variance with the experimental findings, that reported an exponential dependence, and with the text book expression, which predicts an inverse relationship. A new theoretical expression for the osmotic permeability in the single-file file regime is proposed, and several scenarios are discussed to explain both experimental and computational results. The work is expanded to study the combined effects of length and polarity. In varying the polarity, different permeation regimes are uncovered, although no length dependence is found.

In chapter 5 we continue to investigate geometrical determinants of the water permeability. The radius and polarity of model pores were systematically studied, with an emphasis on the transition from and to the single-file regime, their 
relationship with the permeability coefficients and the water pore occupancy. As in the previous chapter, water confined in narrow pores of different polarities displays different permeation regimes, such as the so-called liquid-vapor oscillations. Their connection with the osmotic and diffusive permeability, the water density in the channel and the arrangement of water molecules in the pores are object of this study.

A glance at the thermodynamics of ion permeation In chapter 6 we address the effect of the channel length on the permeation barrier for ions in single-file channels. Because the spontaneous passage of ions is a rare event in the time scales attainable by molecular dynamics simulations, we aim instead at a thermodynamic characterization of the system. Since free energy barriers for ion permeation are related to the observed flux by rate theory, we performed biased simulations to extract the underlying free energy profile for ion permeation. Results indicate that ion permeation in single-file channels is largely influenced by the length of the pore, quite in contrast to water. The total free energy barrier for ion permeation is largely contributed by an entropic penalty, although the overall length dependence is largely enthalpic. These findings, together with the previously established independence of the water osmotic permeability with the channel length, provide novel ways to drive the selectivity for water/ion in natural and model channels. 
1. Introduction 


\section{Theory and Methods}

In this chapter we outline the methodological framework that was applied in this thesis. Further details on the particular methods and setup of the simulations can be found in their respective chapters.

\subsection{Molecular Dynamics principles}

The goal of this thesis requires the characterization of dynamical and thermodynamical properties of condensed phase systems, which involve thousands of degrees of freedom. Due to the large computation cost required, the appropriate computational method is Molecular Dynamics (MD) simulations using a classical force field. In this section the basic principles and approximations of MD simulations are outlined. For a more detailed description, several books [65-67], and reviews [68-70] are available.

\section{Separation of nuclear and electronic degrees of freedom}

Matter is build up of atoms, which combine to form molecules. Therefore, all properties of matter originate from the properties of their constituent atoms and molecules. To our best knowledge, the behavior of the atoms and molecules is dictated by quantum mechanics. In this framework, the time dependent Schrödinger equation fully characterizes all possible states of the system,

$$
i \hbar \frac{\partial \Psi}{\partial t}=\mathcal{H} \Psi
$$

where $\Psi$ is the wave function describing all particles in the system, $h$ is the Planck's constant and $\mathcal{H}$ is the Hamiltonian. Solving equation 2.1 for $\Psi$ provides all the information we can extract from a physical system. For a molecular system, the wave function $\Psi(\mathbf{r}, \mathbf{R})$ depends on the coordinates of all the electrons 
$\mathbf{r}$ and nuclei $\mathbf{R}$. Due to the coupling between all particles, several approximations and simplifications are mandatory to yield the derivation of the system dynamics treatable. The system wave function is expanded as products of electronic, $\psi$, and nuclear, $\varphi$, degrees of freedom. The first simplification involves truncating the expansion in the first term,

$$
\Psi(\mathbf{r}, \mathbf{R})=\sum_{i, j}^{\infty} c_{i} c_{\mathrm{j}} \psi_{i}(\mathbf{r} ; \mathbf{R}) \varphi_{\mathrm{j}}(\mathbf{R}) \approx \psi_{\mathrm{e}}(\mathbf{r} ; \mathbf{R}) \varphi_{\mathrm{n}}(\mathbf{R})
$$

which implies decoupling the electronic and nuclear wave functions. Once the factorization is incorporated in the Schrödinger equation, next simplification involves approximating to zero the change of electronic wave function with respect to the coordinates of the nuclei, $\widehat{\nabla} \psi_{\mathrm{e}}(\mathbf{r} ; \mathbf{R})=0$. Both approximations, commonly known as the Born-Oppenheimer approximation [71, 72], allow us to write the equation for the nuclear degrees of freedom as,

$$
\left(\widehat{T}_{n}+\widehat{V}_{n n}+\mathrm{E}_{e}(\mathbf{R})\right) \varphi_{\mathrm{n}}(\mathbf{R})=V(\mathbf{R}) \varphi_{\mathrm{n}}(\mathbf{R})
$$

where $\widehat{T}_{n}$ and $\widehat{V}_{n n}$ are the kinetic and potenial operators involving the nuclei, and $\mathrm{E}_{e}(\mathbf{R})$ is the energetic contribution from the electrons. Therefore, the potential energy of the complete system in a given configuration, $V(\mathbf{R})$, depends on the positions of the nuclei with a contribution from the electronic structure. This approximation has been shown to hold to a high level of accuracy [73, 74].

The time evolution of the nuclei in a molecular system under the Born-Oppenheimer approximation is dictated by,

$$
i \hbar \frac{\partial \varphi(\mathbf{R}, t)}{\partial t}=\left(\widehat{T}_{n}+\widehat{V}_{n n}+\mathrm{E}_{e}(\mathbf{R})\right) \varphi_{\mathrm{n}}(\mathbf{R}, t)
$$

\section{Classical mechanics provide the equations of motion}

For molecular systems involving thousands of atoms, solving the time dependent equation 2.4 is still not feasible currently. Instead, the nuclei are described by point masses that evolve under the influence of forces acting between them according to Newton's equations of motion,

$$
-\nabla_{i} V(\mathbf{R})=m_{i} \frac{d^{2} \mathbf{R}_{i}(t)}{d t^{2}}
$$


where $V(\mathbf{R})$ is the potential energy, and $\mathbf{R}_{i}$ and $m_{i}$ are the position and masses of atom $i$, respectively. Equation 2.5 is commonly written as $\mathbf{F}_{i}=m_{i} \mathbf{a}_{i}$. A force $\mathbf{F}$ acting on particle $i$ results in its acceleration $\mathbf{a}_{i}$, which modifies the instant velocity $\mathbf{v}_{i}$ and position $\mathbf{R}_{i}$ within a time step $\Delta t$. Except in special cases, an analytical solution of equation 2.5 is not possible. Instead, an iterative numerical procedure known as numerical integration is used to obtain an approximate solution. For a given set of initial coordinates $\mathbf{R}_{0}$ and velocities $\mathbf{v}_{0}$, integration of the equations of motion describes the time evolution of the system, referred to as a trajectory.

Several methods have been developed to numerically solve Newton's equations of motions in discrete time steps, see for example [65]. In this work, the leap-frog modification of the Verlet [75] algorithm was used,

$$
\begin{aligned}
\mathbf{v}\left(t+\frac{\Delta t}{2}\right) & =\mathbf{v}\left(t-\frac{\Delta t}{2}\right)+\frac{\mathbf{F}(t)}{m} \Delta t \\
\mathbf{r}(t+\Delta t) & =\mathbf{r}(t)+\mathbf{v}\left(t+\frac{\Delta t}{2}\right) \Delta t
\end{aligned}
$$

It can be shown that this algorithm is time reversible and has a third order accuracy in $\mathbf{r}$, which is needed to guarantee a correct behavior of the numerical integration, see [76] and references therein. The advantage of this algorithm is that the expensive force calculation has to be done just once per integration step.

\section{The force field approximation}

Solving equation 2.3 to follow the time evolution of a molecular system in the condensed phase is currently limited to relatively small systems and short time scales [77]. A widely popular approximation is to express the potential energy $V(\mathbf{R})$ as a sum of simple and easy-to-compute analytical functions of the nuclear positions. Such functions are postulated and parametrized to reproduce certain reference experimental and/or ab initio quantum chemical calculations. The common term for these potentials and their associated set of parameters is molecular mechanics force fields, or simply force fields.

A typical force field for bio-molecular simulations contains contributions of two kinds: bonded and non-bonded terms. The bonded terms reflect changes in potential energy due to changes in chemical bond length, $V_{b}$, angles between two 
bonds, $V_{a}$, and dihedral torsion around a chemical bond, $V_{d i h}$. For computational efficiency, harmonic potentials are used for the bonds and angles, whereas an harmonic function is used for the dihedral angles. The non-bonded terms represent electrostatic and non-polar van der Waals interactions, the first is computed via Coulomb's law and the latter is modeled by a Lennard-Jones potential. The force field used in this thesis includes the following terms,

$$
\begin{aligned}
V(\mathbf{R}) & =V_{\mathrm{b}}+V_{\mathrm{a}}+V_{\mathrm{dih}}+V_{\text {coul }}+V_{\mathrm{LJ}} \\
& =\sum_{\text {bonds }} \frac{k_{i}}{2}\left(l_{i}-l_{i, 0}\right)^{2} \\
& +\sum_{\text {angles }} \frac{k_{i}}{2}\left(\theta_{i}-\theta_{i, 0}\right)^{2} \\
& +\sum_{\text {dihedrals }} \frac{V_{n}}{2}(1+\cos (n \omega-\gamma)) \\
& +\sum_{\text {atoms i atoms } \mathrm{j}>\mathrm{i}} 4 \epsilon_{i j}\left[\left(\frac{\sigma_{i j}}{r_{i j}}\right)^{12}-\left(\frac{\sigma_{i j}}{r_{i j}}\right)^{6}\right]+\frac{q_{i} q_{j}}{4 \pi r_{i j}}
\end{aligned}
$$

All non-bonded interactions are treated as pair-additive, which means that nonpair additive interactions have to be incorporated by effective pair potentials. The Lennard-Jones potential captures the balance between repulsive short distance electron cloud overlap and long distance induced dipole-dipole attraction (dispersion). Although any of these interactions has a radial dependence encoded in the Lennard-Jones potential, this function is used for computational efficiency and it is successful thanks to global parametrization.

Throughout this thesis, the force field of choice is the OPLS all-atoms (OPLSAA) $[78,79]$, derived for proteins by Jorgensen and coworkers. This force field was designed to work with the TIP4P water model [80].

\section{Simulations in practice}

In order to perform MD simulations successfully, several issues need to be considered: integration time step, boundary conditions, treatment of long-ranged interactions and desired thermodynamic ensemble.

To correctly describe the motions of atoms and molecules using Newtonian mechanics, as opposed to quantum mechanics, the thermal energy has to be 
distinctly larger than the gaps between adjacent quantum energy levels. Since we typically characterize the motions of bonds and angles with classical harmonic potentials, fast vibrations yield very inaccurate dynamics. This problem is avoided by imposing the bonds and angles as constraints in the equations of motion. This has the additional advantage that the integration time step can be increased without loss of integration accuracy [81]. In this work, the iterative LINCS algorithm [82] is used for constraining all bonds. The SETTLE [83] algorithm, designed to analytically solve the equations of motion with 3 constraints, is used for all bonds and angles of the water molecules. The use of constraints allowed a time step of $2 \mathrm{fs}$ for all simulations.

Obviously, only a finite number of particles can be simulated. This implies that accurate modeling of extended condensed phase systems needs to deal with boundary effects. Leaving a hydrated system in vacuum leads to artifacts such as evaporation, high pressure due to surface tension, and preferred orientations of solvent molecules. Several methods [84-86] have been devised to maintain finite boundary conditions by minimizing the artifacts mentioned. Another popular option consists in completely eliminating boundaries by defining a simulation box that is infinitely periodic in space: once a particle leaves the simulation box, it instantaneously reenters from the opposite side. This method, known as periodic boundary conditions (PBC), has a trade-off: periodic conditions impose artificial order in the system. The effect is reduced by enlarging the simulation box, but it is nevertheless present. The PBC simulations are combined with the minimum image convention, where only the closest neighbors are considered for short-ranged non-bonded interactions. In this thesis, PBC are used with a rectangular box for all the simulations. The exact size of the box was adapted to the size of the pore, and was constructed large enough to render only slight artifacts due to the chosen periodic the boundary conditions.

The treatment of non-bonded interactions is the most time-consuming part of the force calculations, which in turn is the most time-consuming step in solving the equations of motion. For $\mathrm{N}$ particles, the computational effort involved scales as $N^{2}$, since each particle interacts with each other particle. A practical approximation is considering that after a given length, termed cut-off length, the interaction between two particles is negligible. For the van der Waals interactions, modeled via the Lennard-Jones potential, the slowest decay is in order of 
$r^{-6}$, which converges quickly. Since the decay of electrostatic energy is exactly $r^{-1}$, long-ranged electrostatic interactions need to be handled with care.

Several strategies have been developed to accurately perform long-range electrostatic calculations. The cut-off approach is known to introduce serious artifacts $[87,88]$, specially for small simulation boxes, so it is currently regarded as an obsolete and error prone methodology. The reaction-field approach [89, 90], routinely used in the GROMOS MD engine [91], treats the space beyond a given cut-off length as a continuum dielectric medium. This dielectric medium responds to changes in the charge distribution within the cut-off. If the boundaries are treated using the $\mathrm{PBC}$ approximation, the Ewald lattice sum is used to achieve convergence of electrostatic calculations. Both reaction-field and lattice methods have advantages and drawbacks [92]. Since a lattice method is used in this thesis, we briefly describe its main features. The Ewald lattice sum method takes advantage of convergence of the short range radial dependence in real space, whereas the long tail of the electrostatic interaction can be added up exactly in Fourier space. Direct calculation scales with $\mathcal{O}\left(N^{2}\right)$. The development of efficient methods to perform Ewald lattice sums, such as Particle Mesh Ewald (PME) [93], or Particle-Particle/Particle-Mesh (PPPM) [94] allowed scaling of $\mathcal{O}(N \log N)$, making these methods affordable. All simulations in this thesis were performed using PME at every time step. A cut-off distance of $1 \mathrm{~nm}$ was used for the Lennard-Jones interactions.

Once a set of potential functions, the boundary conditions and the electrostatic treatment are chosen, we can proceed to integrate Newton's equations of motion using, e.g., equations 2.6-2.7. Trajectories obtained within this formulation generate configurations according to a microcanonical ensemble: the energy, volume and number of particles is constant. Numerical errors, mainly due to finite numerical precision and force truncation, lead to drifts in the total energy of the system. Furthermore, to closely mimic experimental or natural conditions requires the ability to generate ensembles at constant temperature and/or constant pressure. Therefore, there is the need to control the temperature and the pressure during a MD simulation. The Berendsen thermostat [95], used in all simulations in this thesis, couples the system to a given desired temperature $T_{0}$ by means of first order kinetics. Consequently, the relaxation towards the reference temperature is exponential. The velocities of the particles in the sys- 
tem are scaled by $\left(1+\left(\Delta t / \tau_{I}\right)\left(T_{0} / T-1\right)\right)^{1 / 2}$, such that the average temperature is kept constant during a MD simulation of time step $\Delta t$. The time constant of the coupling, $\tau_{I}$, is set to $0.1 \mathrm{ps}$ in all simulations. In the same spirit, the Berendsen barostat [95] rescales the positions of the particles in the simulation box to achieve a preset pressure by coupling the pressure as a first order kinetic equation on the pressure $\mathbf{P}$. When constant pressure simulations were needed, the Berendsen barostat was used with a time constant of 1 ps.

The use of constraints and coupling algorithms imposes corrections in the velocities and positions, which are incorporated in the equations of motion.

\subsection{Derivation of permeation coefficients}

In this section we derive the equations that describe the water flux via osmotic and diffusive permeation through channels of molecular dimensions. We first begin with a general description of the permeability coefficients derived from Finkelstein [5], applicable to the water flux through semipermeable membranes and porous membranes. Second, we derive expressions for the permeability coefficients used in this thesis to extract single-channel permeability coefficients, which are based on several published studies [29, 61, 96-99].

\subsubsection{Definition of the permeability coefficients}

The equivalence between an hydrostatic pressure and an osmotic gradient was demonstrated by van't Hoff [3]. According to the van't Hoff equation, the hydrostatic pressure difference $\Pi$ between two compartments of different concentration $\Delta c_{s}$ (under considerations of ideal dilute solution) separated by a semipermeable membrane is

$$
\Pi=\Delta c_{s} k_{B} T
$$

where $k_{B}$ is the Boltzmann constant and $T$ is the temperature. This equation, derived from equilibrium considerations, implies that in order to maintain different concentrations between two compartments, a hydrostatic pressure $\Pi$ must be exerted. If we assume a linear relationship between pressure and a flow through 
the semipermeable membrane, the expected net flux $J_{v}$ reads,

$$
J_{v}=L_{p}(\Delta P-\Delta \Pi)=L_{p}\left(\Delta P-\Delta c_{s} k_{B} T\right)
$$

where $\Delta P$ is an externally applied hydrostatic pressure. In the absence of such an external pressure, the difference in osmotic pressure alone induces a net flux against the concentration gradient. The proportionality factor $L_{p}$ is the hydraulic permeability, which is related to the osmotic permeability by

$$
P_{f}=\frac{L_{p} R_{g} T}{V_{w} A}
$$

where $V_{w}$ is the water molar volume, $R_{g}$ is the ideal gas constant, and $A$ is the area of the membrane.

To derive the diffusive permeability coefficient $P_{d}$, we express the difference in concentrations not due to different concentrations of solutes, but rather due to different concentrations of labeled solvent (water) molecules $\Delta c_{w}^{*}$ (a gradient of tracer molecules, experimentally deuterated water is used). The molar flux of labeled water molecules $\Phi_{w}^{*}$ is expressed as

$$
\Phi_{w}^{*}=P_{d} A \Delta c_{w}^{*}
$$

If we now assume that the membrane itself is impermeable to water, and that the water flux must occur through $M$ water selective pores embedded in this membrane, then the osmotic and diffusive permeability coefficients can be written as,

$$
\begin{aligned}
& P_{f}=\frac{p_{f} M}{A} \\
& P_{d}=\frac{p_{d} M}{A}
\end{aligned}
$$

where $p_{f}$ and $p_{d}$ are the single-channel osmotic and diffusive permeability coefficients, respectively. The single-channel permeabilities are typically reported in $\mathrm{cm}^{3} / \mathrm{s}$. Since this thesis is concerned with the permeation of water molecules through channels, and not through the membrane, we use the term osmotic and diffusive permeability to denote single-channel permeability coefficients. 


\subsubsection{Permeability coefficients from equilibrium simulations}

From our atomistic simulations, the position of every atom in the system is recorded as a function of time. By combining thermodynamic and rate theory arguments, we can derive equations that allow the extraction of

both the osmotic and diffusive permeability coefficients for a single channel from simulations in the absence of an osmotic gradient.

Single channel diffusive permeability coefficient In the absence of an osmotic or pressure gradient, there is no net flux of water molecules from one compartment to the other. Instead, there is a diffusion exchange of water molecules that are permeated by membrane-embedded channels. This diffusive flux is difficult to observe experimentally, however is straightforward to compute from molecular dynamics simulations. In our computer simulations the water molecules can be effectively labeled, $i$. e., we can follow the motion of individual water molecules. Water molecules exchange between the two compartments via the channel at rates directly proportional to the diffusive permeability coefficient. For a single channel, the diffusive molar flux is

$$
j_{w}^{*}=p_{d} \Delta c_{w}^{*}
$$

Since labeled water molecules diffuse in the same way as the rest of the water molecules, the total number of labeled water molecules crossing the channel in a given direction per time unit $k_{d}$ is proportional to the relative number of tracer molecules between compartments. Setting the concentration of tracers to one of the compartment to zero, the diffusive flux can be written as,

$$
j_{w}^{*}=\frac{c_{w}^{*}}{N_{A} c_{w}} k_{d}=\frac{V_{w} c_{w}^{*}}{N_{A}} k_{d}
$$

where $N_{A}$ is Avogadro's number. Comparing with eq. 2.15, we can finally express the diffusive permeability coefficient as

$$
p_{d}=k_{d} \frac{V_{w}}{N_{A}}=k_{d} v_{w}
$$

where $v_{w}$ is the volume of a water molecule $\left(\sim 30 \AA^{3}\right)$. This result indicates how to extract the diffusive permeability coefficient from a simulation: it is the rate 
at which water molecules completely cross the channel, weighted by the volume of a water molecule.

Single channel osmotic permeability coefficient The fact that non-equilibrium properties, such as an osmotic flux, can be obtained from equilibrium simulations is rooted in the notion that the fluctuations that characterize the system in equilibrium are directly connected to transport properties [29], in the spirit of linear response theory. Combining eq. 2.10 and eq. 2.13, the molar flux established by a gradient concentration through a single channel $j_{w}$ is

$$
j_{w}=p_{f} \Delta c_{s}
$$

In diluted solutions a linear approximation relates the concentration gradient with the difference in chemical potentials [5], $\Delta \mu=-k_{B} T V_{w} \Delta c_{s}$. Therefore, we express eq. 2.18 as

$$
j_{w}=p_{f} \frac{\Delta \mu}{k_{B} T V_{w}}
$$

To derive the osmotic permeability coefficient from equilibrium simulations, we first define a collective coordinate that characterizes the evolution of water molecules along the channel. This collective coordinate $c$ is defined in the interval $[0,1]$ such that a system that evolving in time starts at 0 and ends at 1 , or vice versa, would have effectively translocated one water molecule from one compartment to the other.

If we assume that the diffusive nature of water movement in the pore can be described as hops along the collective coordinate [99], the reduced representation of the system can be thought as an activated process with an associated free energy barrier $\Delta G^{\ddagger}$. In equilibrium, the collective jumps are characterized by equal forward and backward rates , $k_{f}$ and $k_{b}$, respectively. Recalling Kramer's theory of activated processes [100],

$$
k_{0}=k_{f}=k_{b}=\omega_{0} \mathrm{e}^{\beta \Delta G^{\ddagger}}
$$

where $k_{0}$ is defined to indicate the rate in either direction, $\omega_{0}$ is the attempt rate and $\beta=\left(k_{B} T\right)^{-1}$. In the presence of a concentration gradient, the corresponding change in chemical potential lowers the free energy in one compartment with respect to the other by $\Delta \mu$. This results in an increase of the forward rate by 
$\exp (\beta \Delta \mu)$ with respect to the backward rate. This imbalance results in a net molar flux $j_{w}$ that can be related to the equilibrium hopping rate (eq. 2.20) via,

$$
j_{w} N_{A}=\left(k_{f}-k_{b}\right)=k_{0}\left(\mathrm{e}^{\beta \Delta \mu / 2}-\mathrm{e}^{-\beta \Delta \mu / 2}\right)=2 k_{0} \sinh (\beta \Delta \mu / 2)
$$

In a linear approximation, valid for small values of $\Delta \mu$, eq. 2.21 reads

$$
j_{w}=k_{0} \frac{\Delta \mu}{N_{A} k_{B} T}
$$

By comparing eq. 2.19 and 2.22, and recalling that $v_{w}=V_{w} / N_{A}$, we arrive at the result,

$$
p_{f}=k_{0} v_{w}
$$

Eq. 2.23 connects the proportionality between a gradient and a flux, the $p_{f}$, to the equilibrium fluctuations of the water column $k_{0}$. It demonstrates that, provided an appropriate collective coordinate to describe the permeation, we can readily extract osmotic permeability coefficients from equilibrium simulations.

We now discuss two different definitions of the collective coordinate, both used in this thesis, that allow us to compute $k_{0}$, and by virtue of eq. 2.23, also $p_{f}$. In the single-file regime, water molecules permeate in a highly concerted fashion. This concerted motion implies that every hop of the file by a water-water distance $d_{w w}$ effectively displaces one water molecule from one compartment to the other [61]. The collective coordinate is therefore defined as $c=1 / d_{w w}$. In this regime, the intrinsic rate constant of the column $k_{0}$ is computed as the rate at which the water column collectively moves by $d_{w w}$, which can be extracted from the simulations. A mechanistic picture for the calculation of the osmotic and diffusive permeability coefficient is displayed in Fig. 2.1.

An alternative collective coordinate that was used as well in this thesis, proposed originally by Zhu et al. [97], is defined in incremental form as the sum of the displacement of all water molecules along the channel main axis within a time span, normalized by the length of the pore L,

$$
\Delta c(\Delta t)=\frac{1}{L} \sum_{i \in C(\Delta t)} \Delta z_{i}(\Delta t)
$$

where $c(\Delta t)$ is the fraction of the collective coordinate after a time lapse $\Delta t, C(\Delta t)$ 


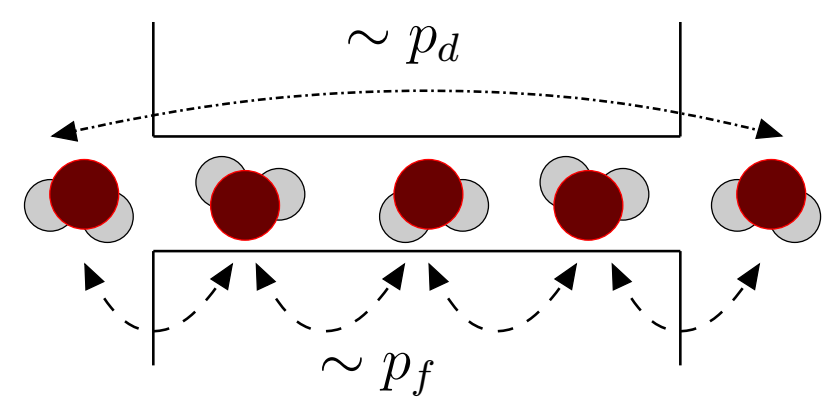

Figure 2.1.: Illustration of the method used to compute permeability coefficients from equilibrium simulations of water permeating a channel in highly collective single-file fashion. The osmotic permeability $p_{f}$ is proportional to the rate of water-water jumps, and the diffusive permeability $p_{d}$ is proportional to the number of water molecules that completely cross the channel.

is the set of water molecules inside the channel during $\Delta t$, and $\Delta z_{i}$ is the displacement along the channel axis $z$ of water molecule $i$. This definition allows to compute a collective coordinate as $c(t)=\left|\sum \Delta c(\Delta t)\right|$. From this coordinate, $k_{0}$ can be obtained as the rate at which transitions between integer values of $c(t)$ take place.

Ratio of osmotic and diffusive permeability coefficients in a single file Finally, a remarkable equality involving the osmotic and diffusive permeability in single-file channels is discussed. A complete translocation of a water molecule requires $n+1$ hops along the channel, where $n$ is the number of water molecules that fits in this channel. As discussed, these hops occur at a rate of $k_{0}$. Correspondingly, the rate at which one water molecule completely crosses the channels is $k_{0} /(n+1)$. Considering this, the ratio of osmotic and diffusive permeability coefficient is equal to,

$$
\frac{p_{f}}{p_{d}}=\frac{k_{0}}{k_{d}}=\frac{k_{0}}{k_{0} /(n+1)}=n+1
$$

This result, originally proposed by Finkelstein [5] as $p_{f} / p_{d}=n$, has been derived by means of several formalisms $[31,96,99,101]$. In this thesis, the range of validity of this expression is systematically investigated. 


\subsection{Free energies and molecular dynamics simulations}

A system in which the temperature, the volume and the number of particles are kept fixed is described by the canonical thermodynamic ensemble. Here we consider that the interactions between all $N$ particles in the system are described by a classical Hamiltonian, consisting of the kinetic energy depending on the momenta $\mathbf{p}_{i}$ of the particles and potential energy that depends on the positions of the all particles $\mathbf{x}^{N}$,

$$
\mathcal{H}\left(\mathbf{p}^{N}, \mathbf{x}^{N}\right)=\sum_{i=1}^{N} \frac{\mathbf{p}_{i}^{2}}{2 m_{i}}+V\left(\mathbf{x}^{N}\right)
$$

The associated partition function is then [102],

$$
\mathcal{Z}_{N}(T, V)=\int \mathrm{d}^{N} \mathbf{x} \mathrm{d}^{N} \mathbf{p} \mathrm{e}^{-\beta \mathcal{H}\left(\mathbf{p}^{N}, \mathbf{x}^{N}\right)}
$$

and the probability $\mathcal{P}$ to find the system in a particular configuration $\left\{\mathbf{x}^{N}, \mathbf{p}^{N}\right\}$ is

$$
\mathcal{P}\left(\left\{\mathbf{x}^{N}, \mathbf{p}^{N}\right\}\right)=\frac{\mathrm{e}^{-\beta \mathcal{H}\left(\mathbf{p}^{N}, \mathbf{x}^{N}\right)}}{\mathcal{Z}_{N}}
$$

The partition function of the system is connected to the Helmholtz free energy via

$$
F(T, V, N)=-k_{B} T \ln \mathcal{Z}_{N}(T, V)
$$

An important consideration is that when a system of $\mathrm{N}$ particles is in a state in which the temperature and the volume are constant, the system spontaneously evolves to minimize the (Helmholtz) free energy. The free energy is the driving force of the system, it discriminates whether changes in the configuration of the system are spontaneous or require an external amount of energy to be realized. Although all the derivations here are carried out for the Helmholtz free energy, the calculations to obtain the Gibbs free energy in the case of an isobaric ensemble are formally equivalent.

Constrained free energies As seen in eq. 2.27, the free energy depends on all degrees of freedom of the system, $i$. e., is a multivariate function. In many cases, 
however, it is desirable to have a formulation that allows to quantify the impact of only a subset of parameters on the free energy. A constrained free energy is defined exactly in this manner [103], it expresses the value the free energy of the whole system when a given observable of the system $\mathcal{A}(\mathbf{p}, \mathbf{x})$ is constrained to a given generalized coordinate $\xi$. By integrating out the rest of degrees of freedom, the constrained partition function is

$$
\mathcal{Z}(\xi)=\int \mathrm{d}^{N} \mathbf{x} \mathrm{d}^{N} \mathbf{p} \delta(\mathcal{A}(\mathbf{x}, \mathbf{p})-\xi) \mathrm{e}^{-\beta \mathcal{H}\left(\mathbf{p}^{N}, \mathbf{x}^{N}\right)}
$$

where $\delta$ is the Dirac $\delta$-function. The corresponding constrained free energy $\mathcal{F}$ is then obtained by

$$
\beta \mathcal{F}(\xi)=-\ln \mathcal{Z}(\xi)
$$

The probability to find the system in the state described by $\xi$ is

$$
\mathcal{P}(\xi)=\frac{\mathcal{Z}(\xi)}{\mathcal{Z}_{N}}=\frac{1}{\mathcal{Z}_{N}} \int \mathrm{d}^{N} \mathbf{x} \delta(\mathcal{A}(\mathbf{x}, \mathbf{p})-\xi) \mathrm{e}^{-\beta V(\mathbf{x})}
$$

which can be rationalized as follows: the ratio between the phase space volume available for an observable constrained at $\xi$ and the total available phase space is exactly the probability that the system is found at $\xi$. Eq. 2.32 and 2.31 provide a direct connection between the $\mathcal{F}(\xi)$ and our molecular dynamics simulations,

$$
\beta \mathcal{F}(\xi)=-\ln \mathcal{P}(\xi)+\beta F
$$

which expresses the constrained free energy as the probability to find the system in $\xi$ and a contribution from the total free energy, which is constant. Since we are interested in how the constrained free energy changes when the system evolves along $\xi$, the unknown term $\beta F$ is not needed.

A potential of mean force is a constrained free energy A useful constrained free energy is obtained by defining $\mathcal{A}(\mathbf{x}, \mathbf{p})=\mathbf{x}_{1}$, fixing a given particle to the position $\mathbf{x}_{1}$. The free energy required to move the particle from a reference state to the position $\mathbf{x}_{1}$ is known as the potential of mean force (PMF) [104], and we denote it by $\mathcal{W}\left(\mathbf{x}_{1}\right)$. The PMF along a given pathway for $\mathbf{x}_{1}$ generates a free energy profile, which is useful for a reduced representation of the dynamics of a system along that pathway. The PMF is, e.g., a basic ingredient for the 
Generalized Langevin Equation [105], which describes the dynamic evolution of a given subset of the total degrees of freedom. The PMF can be expressed as

$$
\mathrm{e}^{-\beta \mathcal{W}\left(\mathbf{x}_{1}\right)}=C \int \mathrm{d} \mathbf{x}_{2} \cdots \mathrm{d} \mathbf{x}_{N} \mathrm{e}^{-\beta V\left(\mathbf{x}_{1}, \mathbf{x}_{2}, \ldots, \mathbf{x}_{N}\right)}
$$

where the constant $\mathrm{C}$ denotes a reference state. The integration is done over all the positions of the particles except the one of interest, $\mathbf{x}_{1}$. The name of potential of mean force for this kind of constrained free energies is due to the fact that the derivative of $-\mathcal{W}\left(\mathbf{x}_{1}\right)$ with respect to $\mathbf{x}_{1}$ is the mean average force exerted on the particle 1 located at position $\mathbf{x}_{1}$,

$$
-\frac{\partial \mathcal{W}\left(\mathbf{x}_{1}\right)}{\partial \mathbf{x}_{1}}=\left\langle\mathbf{F}\left(\mathbf{x}_{1}\right)\right\rangle
$$

This equation provides another link between molecular dynamics simulations and constrained free energies, in this case $\mathcal{W}\left(\mathbf{x}_{1}\right)$. By integrating the average force exerted on particle $\mathbf{x}_{1}$ from a reference state $\mathbf{x}_{1}^{\prime}$ we obtain the free energy difference between these two states.

\subsubsection{Methods to extract free energies from simulations}

Extracting absolute free energies via eq. 2.29 from simulations involving thousands of atoms is challenging since it requires the calculation of $\mathcal{Z}_{N}$, which is typically prohibitive in a high dimensional phase space. However, for most practical applications it suffices to determine the relative free energy between two states, e.g., the free energy difference between an ion in the central channel position and the ion in the bulk. Relative constrained free energies can be readily computed from molecular dynamics simulations by employing eq. 2.32 for each state of interest and computing the difference,

$$
\Delta \mathcal{F}=-k_{B} T \ln \frac{\mathcal{P}(\xi)}{\mathcal{P}\left(\xi^{\prime}\right)}
$$

When dealing with the permeation of atoms and molecules through a straight channel that has its main axis oriented parallel to the $z$ axis, a convenient choice for $\mathbf{x}_{1}$ is $z_{1}$, the $\mathrm{z}$ coordinate of the reference atom (or the $\mathrm{z}$ component of center of mass of the molecule). Since we would like to obtain a position-dependent 
constrained free energy profile, we work with the potential of mean force, previously introduced. Also for convenience, we define our potential of mean force with respect to a reference point $z_{0}$ located in the bulk. In cartesian coordinates, we express the potential of mean force as

$$
\mathrm{e}^{-\beta \mathcal{W}(z)}=\mathrm{e}^{-\beta \mathcal{W}\left(z_{0}\right)} \frac{\int \mathrm{d} x \int \mathrm{d} y \mathrm{e}^{-\beta V(x, y, z)}}{\int \mathrm{d} x \int \mathrm{d} y \mathrm{e}^{-\beta V\left(x, y, z_{0}\right)}}
$$

The integration carried out for $x$ and $y$ needs to be bound to be meaningful. This is guaranteed for the atoms or molecules inside the channel, but not when they are in the bulk. To ensure a well defined reference state, in our calculations we restrict the integration outside the channel to an area equal to the are of the channel. This also ensures a well defined concentration of channels, which allows direct comparison between different systems. We finally write

$$
\Delta \mathcal{W}(z)=-k_{B} T \ln \frac{\mathcal{P}(z)}{\mathcal{P}\left(z_{0}\right)},
$$

where the notation $\Delta \mathcal{W}(z)$ implicitly assumes that a reference state at $z_{0}$ is used to define the difference in (constrained) free energy. The relative probability to find a particle at position $z$ with respect to position $z_{0}$ can be extracted from simulations if the system is able to sample all degrees of freedom. For PMF profiles with high barriers this is impractical, since the probability to find the atom/molecule decreases exponentially with the barrier height. In this thesis, this method was used to compute the PMF profile for single water molecules along the channel. The typical value for the free energy barrier in this cases is $\sim 3.2 k_{B} T$, which translates into a relative probability of $4 \cdot 10^{-2}$. However, it is not a valid approach to compute the free energy barrier for ion permeation, since they are typically in the order of $\sim 15 k_{B} T$, which translates into a very low relative probability, $\sim 10^{-7}$, too low to rely on spontaneous events.

There are several methods proposed to circumvent the lack of sampling in regions inaccessible due to its very low probability. Most of these methods overcome this limitation by guiding or confining the system along a given reaction coordinate. The external bias is designed such that its effect on the underlying free energy can be removed or accounted for. 
Free energies from enforced equilibrium simulations In transitions between states that occur infinitely slow, the work $W$ performed by (or against) the system is exactly the free energy difference $(W=\Delta F)$. Since a free energy is a state function, several methods use conveniently chosen reaction coordinates to drive the system from state A to state B. By performing this transition infinitely slow ${ }^{1}$, and recording either the mean force or a probability distribution, we can calculate $\Delta F$. A potential of mean force $\mathcal{W}\left(\mathbf{x}_{1}\right)$ can also be obtained if the transitions $\mathrm{A} \rightleftharpoons \mathrm{B}$ represent a succession of states located infinitely close along the reaction coordinate. Here we present a brief list of the most popular methods,

- Free energy perturbation [106, 107] (FEP) and thermodynamic integration [104] (TI). These methods typically provide free energy difference rather than a potential of mean force. Multiple variants of the originally proposed methods have been developed in order to improve their efficiency [108]. These methods are typically used to compute free energies related to chemical modifications, such as mutations in the sequence of a protein. Such transformation usually require unphysical reference states that are used to build a thermodynamic cycle, from which relative free energies are extracted (for this reason this methods are also termed "computational alchemy" [107]).

- Particle insertion method [109]. This method uses 'ghost' particles to randomly probe the interaction energy a real particle would have in selected configurations. It can be used, e. $g$., to compute the free energy cost of inserting a water molecule in specific locations of a lipid membrane.

- Constrained force simulations $[110,111]$. The system is simulated at discrete positions along the reaction coordinate, and displacements from the reference position are avoided by continuously resetting the particle to its predefined position. The average forces exerted on the particle are recorded, an integration along the reaction coordinate yields a (discrete) PMF.

- Umbrella sampling [112]. The position of the particle along the reaction coordinate is confined around predefined positions by an external potential. The position along the reaction coordinate and the strength of the external

\footnotetext{
${ }^{1}$ As slow as possible for practical applications.
} 
potential are chosen to ensure that the system completely samples the reaction coordinate. This method is used in this thesis, and it is introduced in more detail shortly, together with the methodology used to recover the underlying PMF from the biased simulation.

Free energies from non-equilibrium simulations The work performed when the transition between different states of the system is not carried out under conditions of equilibrium is due to the free energy difference and a dissipative term, $W=\Delta F+W_{\text {diss }}$. To compute the free energy from the work distribution, the Jarzynski equality [113], a special case of the Crooks theorem [114] is typically used. The Jarzinski equation,

$$
\mathrm{e}^{-\beta \Delta F}=\left\langle\mathrm{e}^{-\beta W}\right\rangle
$$

where the $\langle\cdot\rangle$ denotes the ensemble average of the work under non-equilibrium conditions, allows to compute free energies from multiple non-equilibrium simulations, each contributing a work of $\mathrm{W}$ to the ensemble average. Direct application of eq. 2.39 is however impractical, since it requires the sampling of regions of the probability distribution $P(W)$ with negative dissipative work, which are very rare. To alleviate this problem, a common approach to obtain the free energy is to use a cumulant expansion of $\left\langle\mathrm{e}^{-\beta W}\right\rangle$ up to second order $[106,113,115]$, such that the free energy reads

$$
\begin{aligned}
\Delta F & =\langle W\rangle-1 / 2 \beta\left\langle(W-\langle W\rangle)^{2}\right\rangle \\
& =\langle W\rangle-1 / 2 \beta \sigma_{W}^{2},
\end{aligned}
$$

where $\sigma_{W}$ is the variance of the work distribution $P(W)$ and $\langle W\rangle$ is the average work.

Another possibility is the use of the transient fluctuation theorem [114, 116, 117], which connects the work distribution of forward and backward transitions between state $\mathrm{A}$ and state $\mathrm{B}$ to the dissipative work (note that $W-\Delta F=W_{\text {diss }}$ ),

$$
\frac{\mathcal{P}(W)\{A \rightarrow B\}}{\mathcal{P}(W)\{A \leftarrow B\}}=\mathrm{e}^{-\beta(W-\Delta F)}
$$

from eq. 2.41 is follows that the value of $W$ for which the two probability distri- 
butions, $\mathcal{P}(W)\{A \rightarrow B\}$ and $\mathcal{P}(W)\{A \leftarrow B\}$ are equal corresponds to the free energy difference $\Delta F$ between these two states.

Applications that require the use of non-equilibrium methods are, e.g., forceprobe molecular dynamics (FPMD) simulations [118], where the system of interest is guided via an harmonic potential. During the simulation, the harmonic potential is moved along the reaction coordinate, and the mechanical work is recorded. By performing multiple FPMD simulations a work distribution $P(W)$ is collected, which can be translated into a free energy by means of eq. 2.39 or eq. 2.40. Alternatively, several FPMD simulations can be performed from $\mathrm{A} \rightarrow \mathrm{B}$ and from $\mathrm{B} \leftarrow \mathrm{A}$, and use eq. 2.41 to obtain $\Delta F$. Another method that requires non-equilibrium equalities is fast-growth TI $[119,120]$, since the transitions between states are enforced without allowing sufficient relaxation times for the system, as opposite to the traditional (slow-growth) TI.

Umbrella sampling A widely used approach to deal with rare events, such as the permeation of ions through narrow channels, is to enforce the system to sample all the positions along a previously defined reaction coordinate $\xi$, which is discretized in a number of bins $N_{w}$ with reference positions at $\xi_{i}$. The method known as umbrella sampling (US), proposed by Torrie and Valleau [112] ${ }^{2}$, uses an external biasing potential $w_{i}$ to confine the atom/molecule around a preestablished position $\xi_{i}$. The relative probabilities within the regions sampled are collected, and a correction is included to account for the external bias prior to obtaining $\mathcal{W}(\xi)$. A common form of the biasing potential $w_{i}$ is a harmonic potential,

$$
w_{i}(\xi)=\frac{1}{2} k\left(\xi-\xi_{i}\right)^{2},
$$

which restrains the atom/molecule of interest in the vicinity of $\xi_{i}$. Therefore, the potential energy acting on a particle restrained at $\xi_{i}$ is due to $V(\mathbf{x})+w_{i}(\xi)$. The probability to find the particle at position $\xi$ due to the bias at $\xi_{i}$ is

$$
\mathcal{P}_{i}^{\text {bias }}(\xi)=\frac{\int \mathrm{d}^{N} \mathbf{x} \delta(\xi(\mathbf{x})-\xi) \mathrm{e}^{-\beta\left(V(\mathbf{x})+w_{i}(\xi)\right)}}{\int \mathrm{d}^{N} \mathbf{x} \mathrm{e}^{-\beta\left(V(\mathbf{x})+w_{i}(\xi)\right)}}
$$

\footnotetext{
${ }^{2}$ Here we follow the derivations from Roux [121] rather than the one presented in the original paper of Torrie and Valleau.
} 
which can be rewritten as,

$$
\mathcal{P}_{i}^{\text {bias }}(\xi)=\mathrm{e}^{-\beta w_{i}(\xi)} \frac{\int \mathrm{d}^{N} \mathbf{x} \delta(\xi(\mathbf{x})-\xi) \mathrm{e}^{-\beta V(\mathbf{x})}}{\int \mathrm{d}^{N} \mathbf{x} \mathrm{e}^{w_{i}(\xi)} \mathrm{e}^{-\beta V(\mathbf{x})}},
$$

or:

$$
\mathcal{P}_{i}^{\text {bias }}(\xi)=\mathrm{e}^{-\beta w_{i}(\xi)} \frac{\mathcal{P}_{i}(\xi)}{\left\langle\mathrm{e}^{\left.-\beta w_{i}\left(\xi_{i}\right)\right\rangle}\right.}
$$

where $\langle\cdot\rangle$ is a short hand notation for the ensemble average of $\mathrm{e}^{w_{i}(\xi)}$, and $\mathcal{P}(\xi)$ is the constrained probability of the system in the absence of bias. By obtaining $\mathcal{P}(\xi)$ we can extract the potential of mean force by means of eq. 2.38 up to an additive constant due to a reference state $\mathcal{P}\left(\xi_{0}\right)$. More explicitly,

$$
\mathcal{W}(\xi)=-k_{B} T \ln \frac{\mathcal{P}(\xi)}{\mathcal{P}\left(\xi_{0}\right)}-w_{i}(\xi)+F_{i}+\mathcal{W}\left(\xi_{0}\right)
$$

where we have defined $F_{i}=\left\langle\mathrm{e}^{-\beta w_{i}\left(\xi_{i}\right)}\right\rangle$. Apart from the arbitrary reference $\mathcal{W}\left(\xi_{0}\right)$ and $F_{i}$, we can extract all the ingredients needed from the biased simulations. The constant $F_{i}$ reflects the free energy required to introduce the bias into the system. In order to construct the $\operatorname{PMF} \mathcal{W}(\xi)$ from the simulation, $F_{i}$ is required.

Using WHAM to reconstruct the unbiased probability density Although several methods have been proposed to determine $F_{i}$, the method that is currently considered the most suitable (according to Roux [121]) is the so-called weighted histogram analysis method (WHAM)[122]. From $N_{w}$ umbrella sampling simulations along the reaction coordinate $\xi$, the WHAM method expresses the overall bias-free profile of probability distribution $\mathcal{P}(\xi)$ as a weighted sum over the individual bias-free probability distributions $\mathcal{P}_{i}(\xi)$ by minimizing the statistical error in recombining all $\mathcal{P}_{i}(\xi)$. The overall probability is expressed as,

$$
\mathcal{P}(\xi)=\sum_{i=1}^{N_{w}} \mathcal{P}_{i}(\xi) \frac{n_{i} \mathrm{e}^{-\beta\left(w_{i}(\xi)-F_{i}\right)}}{\sum_{j=1}^{N_{w}} n_{j} \mathrm{e}^{-\beta\left(w_{j}(\xi)-F_{j}\right)}},
$$


where $n_{i}$ is the number of points in the histogram used to construct $\mathcal{P}_{i}(\xi)$. Using eq. 2.45 and $F_{i}=\left\langle\mathrm{e}^{-\beta w_{i}\left(\xi_{i}\right)}\right\rangle$ we obtain

$$
\mathcal{P}(\xi)=\sum_{i=1}^{N_{w}} \mathrm{e}^{\beta w_{i}(\xi)} \mathcal{P}_{i}(\xi) \mathrm{e}^{-\beta F_{i}} \frac{n_{i} \mathrm{e}^{-\beta\left(w_{i}(\xi)-F_{i}\right)}}{\sum_{j=1}^{N_{w}} n_{j} \mathrm{e}^{-\beta\left(w_{j}(\xi)-F_{j}\right)}}
$$

The WHAM equations are

$$
\begin{aligned}
\mathcal{P}(\xi) & =\sum_{i=1}^{N_{w}} \mathcal{P}_{i}(\xi) \frac{n_{i} \mathrm{e}^{-\beta\left(w_{i}(\xi)-F_{i}\right)}}{\sum_{j=1}^{N_{w}} n_{j} \mathrm{e}^{-\beta\left(w_{j}(\xi)-F_{j}\right)}} \\
\mathrm{e}^{-\beta F_{i}} & =\int \mathrm{d} \xi \mathrm{e}^{-\beta w_{i}(\xi)} \mathcal{P}(\xi)
\end{aligned}
$$

These two equations are solved in a self-consistent manner for $F_{i}$. In practice, this is achieved by formulating an initial guess for $F_{i}$ and iterating the WHAM equation until the difference between $\left|F_{i}-F_{i}^{\text {last }}\right|$ is below a given threshold. 
2. Theory and Methods 


\section{Gramicidin channels: a starting point}

Gramicidin A and its derivative has been the prototypical selective ion channel for biophysicists and physiologist for the last 40 years. Although water permeation has not been neglected [61, 123-125], the main focus of study has been its ion conductance and ion selectivity. Here we start our study of the connection between water flux and structural properties by analyzing the water transport through gramicidin A and a cross-linked gramicidin derivative.

\subsection{Gramicidin and midigramicidin}

To contribute to the understanding of the interplay between the physicochemical characteristics of a peptidic nanopore and its water permeability, we start by considering the well known channel forming peptide gramicidin A (gA) (Fig. 3.1). In this study, we selected the head-to-head helical dimer conformation (gA-HD), since it is generally accepted as the dominant form in lipid environments [126128]. The peptide was studied in a model lipid membrane, dimyristoylphosphatidylcholine (DMPC), since water permeability studies are available for this membranes.

To expand the spectrum of physicochemical characteristics, a second channel forming peptide was considered. To this end, the covalently-linked asymmetric peptide midigramicidin (MDg) was studied (Fig. 3.1), also embedded in a DMPC bilayer. Midigramicidin has gramicidin A as one of the two linked aminoacid sequences. The other subunit is also based on gramicidin A, but was shortened by four residues at the N-terminus. The overall sequence, almost identical to the gA, induces a $\beta$-helix fold of the peptide once embedded in the membrane [129, 130], structurally equivalent to the gA-HD fold. Due to the difference in the number 
of residues, MDg channels are shorter than gA-HD channels $(\sim 2.4 \mathrm{~nm}$ for gA$\mathrm{HD}, \sim 2.2 \mathrm{~nm}$ for $\mathrm{MDg}$ ). An additional advantage of the selected channels is that both the structure of gA-HD and MDg are available from NMR studies [129, 131], therefore providing a reasonable starting configuration for our simulations.
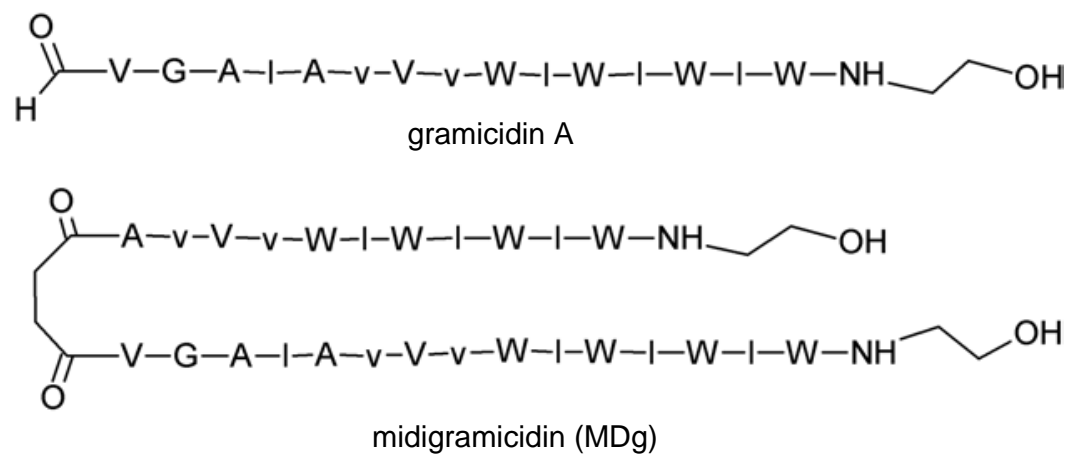

Figure 3.1.: Sequences of gramicidin A ( $g A)$ and cross-linked midgramicidin (MDg).

In the following, the stability of the peptidic channels embedded in DMPC bilayers, the interplay between the behavior of the peptides and the membrane structure, and the connection to water permeability are discussed. Finally, modifications of the gramcidin A channel towards a more efficient single-file water permeation in lipid environments will be suggested, based on the presented simulation results.

Setup of the simulation systems Initial structures for $\mathrm{gA}$ in the helical dimer (gA-HD) conformation and the linked MDg were taken from protein database entries 1MAG [131] and 1TKQ [129], respectively. After energy minimization in vacuum, the channels were embedded in a previously equilibrated dimyristoylphosphatidylcholine lipid bilayer containing 124 lipids, solvated by $\sim 3600$ water molecules. The simulation box was then replicated in $z$ direction, normal to the lipid plane. Sodium and chloride ions were added to one of the water compartments to achieve a concentration of $1 \mathrm{M}$. To insert the channels in the DMPC bilayer, an initial cylindrical hole was made by removing two lipids in the central position from each layer. A molecular surface of the gA-HD was used to define a region from which the lipid molecules should be expelled. A modified version of the GROMACS simulation package was used to expel the lipids by means of force normal to the molecular surface [132]. After a cavity was formed 
in the bilayer, the peptide was finally incorporated and an energy minimization was carried out with the peptidic backbone kept fixed. The same process was repeated for midigramicidin.

a)

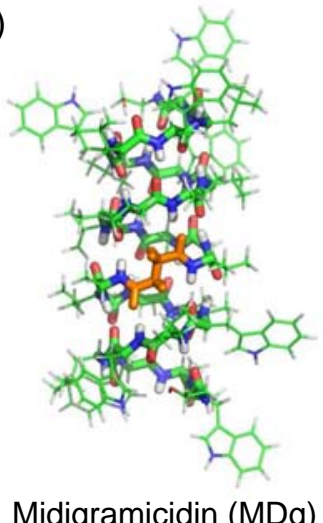

Midigramicidin (MDg)

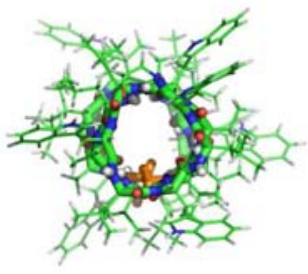

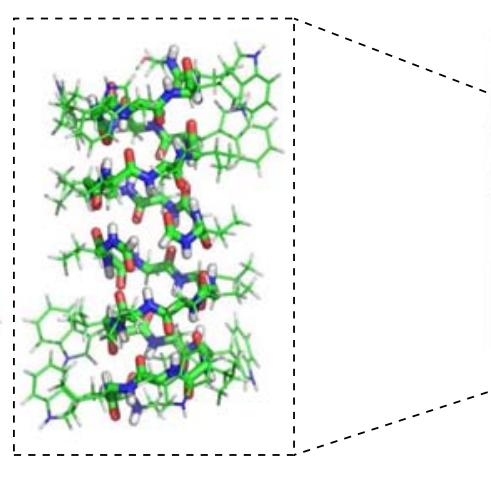

Gramicidin A (gA-HD)

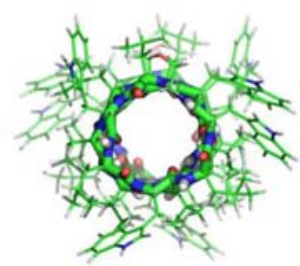

b)

Figure 3.2.: a) Top and side view of the midigramicidin $(M D g)$ and the helical dimer gramicidin A ( $g A-H D)$ peptidic channels. The succinyl linker in the $M D g$ is highlighted in orange. b) Double bilayer setup for gA-HD embedded in DMPC. The lipid head groups are depicted as dark gray balls, and their tails as gray sticks. The water molecules are displayed as white and red sticks.

After the insertion of the peptides in the membrane, the backbone of the peptides was kept fixed by means of position restraints to equilibrate the interface between the channel and the membrane. After 2 ns of molecular dynamics simulations, the position restraints were removed and the channels were allowed to freely diffuse within the membrane. The simulation time for gA-HD amounted to $100 \mathrm{~ns}$, and to $90 \mathrm{~ns}$ for $\mathrm{MDg}$.

As a control system, a hydrated DMPC bilayer containing 128 lipid molecules and $\sim 3600$ water molecules was simulated for $18 \mathrm{~ns}$.

Interactions between all atoms were described by means of the OPLS allatom force field [78, 79], and water molecules were described by the TIP4P model [80]. The DMPC force field parameters were taken form Berger et al. [133]. 
All simulations were performed using the GROMACS 3.3.1 simulation software $[134,135]$. Electrostatic interactions were calculated with the particle mesh Ewald method $[93,136]$. Short-range repulsive and attractive dispersion interactions were simultaneously described by a Lennard-Jones potential, using a cut-off length of $1.0 \mathrm{~nm}$. The Settle [83] algorithm was used to constrain bond lengths and angles of water molecules, and LINCS [82] was used for all other bonds and angles, allowing a time step of $2 \mathrm{fs}$. The temperature in the simulations was kept constant by separately coupling the peptide, the lipid molecules, and the water molecules together with the ions to an external heat bath at $300 \mathrm{~K}$ [95] $(\tau=$ $0.1 \mathrm{ps}$ ). The pressure in the simulations was kept constant by a weak anisotropic coupling $(\tau=1 \mathrm{ps})$ to a pressure bath of $1 \mathrm{~atm}$. Average pore radii were computed using the program HOLE [137].

Permeability coefficients Osmotic permeability coefficients were estimated from the molecular dynamics trajectories by means of the collective approach described in Sec. 2.2. Diffusive permeability coefficients were computed as the rate of water molecules completetely crossing the channel multiplied by the volume of a water molecule, also detailed in Sec. 2.2.

\subsection{Results and discussion}

Structural stability, mismatch, tumbling and lipid-channel interaction The structural stability of the gA-HD and MDg was checked by monitoring the time evolution of the root mean squared displacement (RMSD), shown in Fig. 3.3. The RMSD was computed with respect to all the backbone atoms after translational and rotational fitting to their starting structure. After a quick initial increase, the RMSD oscillates around their mean value, corresponding to the averaged structure generated by the force field. Both gA-HD and MDg structures are stable during the simulation time. The gA-HD conformation shows larger fluctuations than the MDg, presumably caused by the extra structural stability in $\mathrm{MDg}$ due to the succinyl linker. The average force field structures have an effective pore radius of $0.14 \mathrm{~nm}$ for $\mathrm{gA}-\mathrm{HD}$ and $0.15 \mathrm{~nm}$ for $\mathrm{MDg}$.

For gA-HD, both the N-terminal and C-terminal capping groups (formyl and ethanolamine, respectively) frequently deviate from their original positions. The 


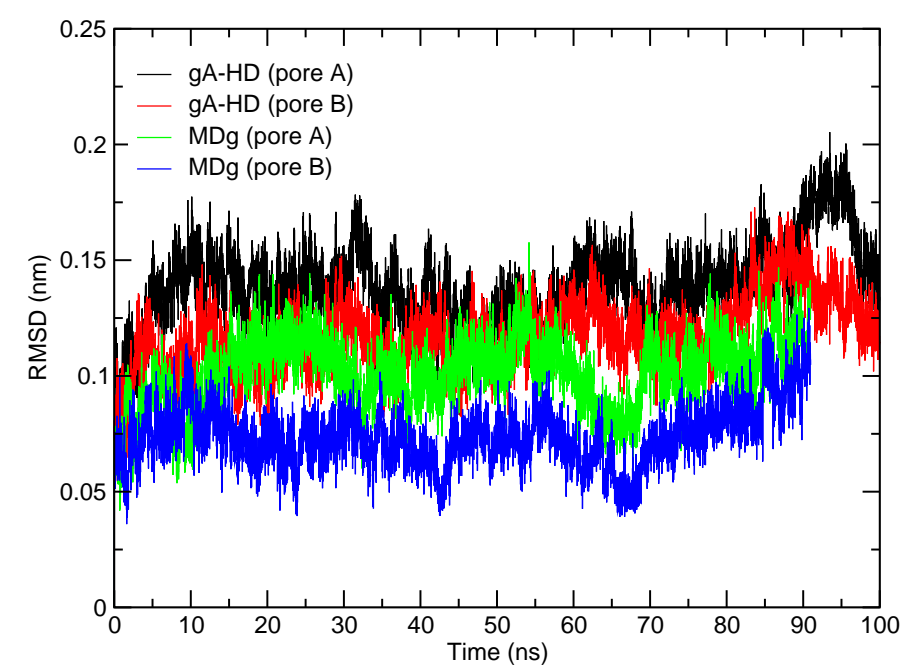

Figure 3.3: RMSD of the backbone atoms for the four studied channels, two gA-HD and two $M D g$, with respect to their initial structure as function of the simulation time.

ethanolamine groups tend to leave their peptidic carbonyl hydrogen bond partner in favor of interactions with water molecules at the lipid/bulk interface, which occasionally blocks the channel. This effect was also seen in the midigramicidin system. Likewise, the formyl groups were observed $(\sim 10 \%$ of the simulation time) to bend towards the channel lumen, establishing hydrogen bonds with water molecules from the bulk/lipid interface. Although such disturbances do not completely block the channels, they interfere with permeating water molecules. Since midigramicidin is linked at the N-terminus, the formyl group is not present and therefore this behavior is not possible.

The pure DMPC bilayer simulated has an average thickness of $\sim 4 \mathrm{~nm}$, computed as the average distance between the center of mass of the head groups under the simulated conditions. This thickness is much larger than the length of the embedded channels $(\sim 2.4 \mathrm{~nm}$ for $\mathrm{gA}-\mathrm{HD}, \sim 2.2 \mathrm{~nm}$ for $\mathrm{MDg})$. However, the hydrophobic lipid chains are shorter, $\sim 2.2 \mathrm{~nm}$ and therefore are about the right length to match the peptides. The amphiphilic tryptophan residues of gA-HD and MDg peptides tend to remain at the interface between the lipid tails and the solvated lipid head groups. The closest distance between the tryptophan residues located at the extremes of the channel is $1.2 \mathrm{~nm}$ in $\mathrm{gA-HD}$, and $1 \mathrm{~nm}$ in MDg. Therefore, in order to allow the tryptophan to position in the polarity interface, either to establish hydrogen bonds between the $\mathrm{NH}$ of the indole group and interfacial water molecules $[45,138-140]$ or the glycerol oxygens of the DMPC, the membrane has to locally deform [141-143]. This driving force affects the dynamics of the peptides and the lipid bilayer: the membrane thickness is 
locally altered with respect to a pure bilayer, and the peptides tilt with respect to the bilayer normal. Both effects were observed during our molecular dynamics simulations.
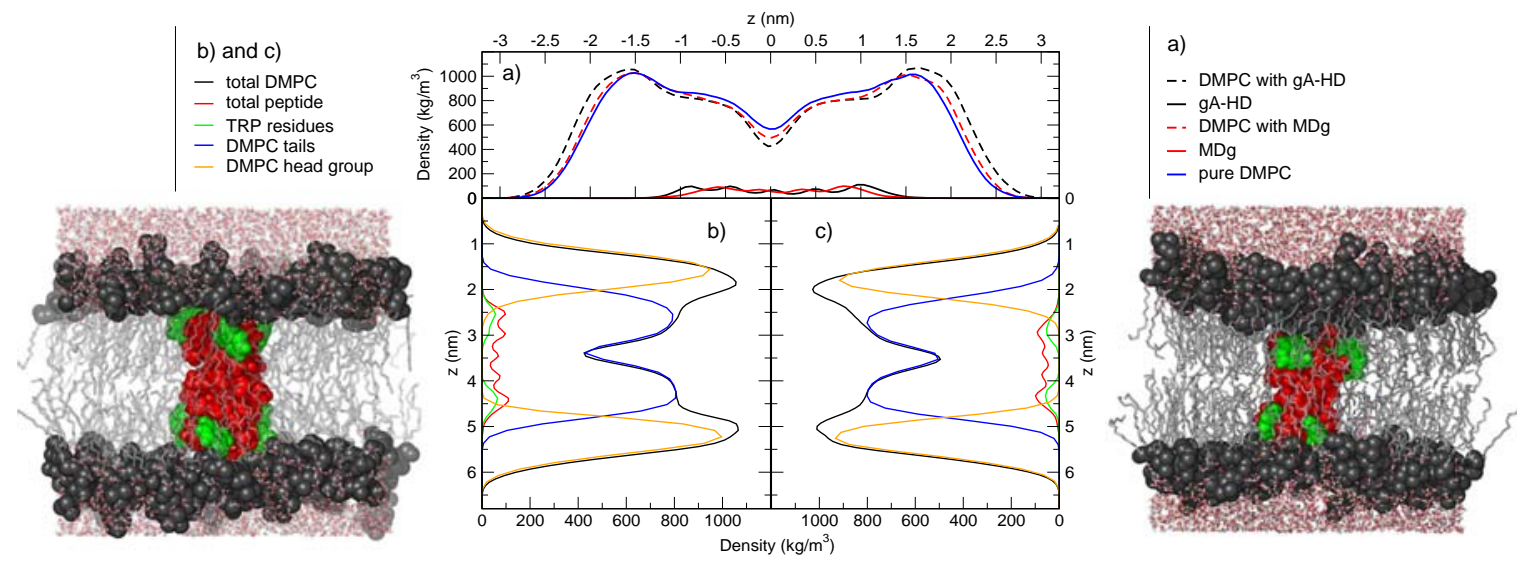

Figure 3.4.: Mass density of the DMPC lipids and the peptidic channels along the membrane normal averaged over the length of the simulation. a) Comparison between the mass density profile for gA-HD - DMPC, MDg - DMPC and pure DMPC systems. b) gA-HD - DMPC and b) $M D g$ - DMPC mass density decomposed in contributions from the lipids (black), the lipid head groups(orange), the lipid tails (blue), the peptides (red) and the tryptophan residues of the peptides (green). The drawing on the side show snapshots of the simulated system displaying one of the two bilayers in the systems. The peptides are drawn as red balls, with the tryptophan residues highlighted in green. The lipid head groups are represented by dark gray balls, and the lipid tails by gray sticks. The water molecules are displayed as white and red sticks. The hydration layers are, in fact, larger than shown due to the periodic boundaries.

Figure 3.4 shows the averaged mass density of the DMPC bilayer and of the peptidic channels as function of the axis normal to the lipid bilayer, for both gAHD and MDg systems. The densities were decomposed into contributions from the tryptophan groups, the lipid tails and the lipid head groups. The density profiles show that, on average, the channels remain centered in the bilayer, such that the tryptophan residues are located at the interface between the lipid tails and the head groups. The longer peptide, gA-HD, causes the membrane to thicken by about $0.25 \mathrm{~nm}$, depleting the density at the middle of the bilayer. MDg shows a smaller reduction of the density at the center of the bilayer, and almost no average thickening of the membrane. Since the densities are averaged over slices parallel to the membrane plane, the profiles depend on the molecular ratio between peptide and lipid molecules, in our case 1:120. Larger amounts of excess lipids would have averaged out the effect of the peptide. 
To further characterize the effect of the peptides on the lipidic membrane, we analyzed the local deformation of the membrane around the embedded channels. We used the distance between the central carbon atoms of the glycerol residues of opposing DMPC layers to monitor the change of the thickness of the bilayer. In a pure DMPC bilayer, the mean value of the thickness computed with these criteria is $\sim 3 \mathrm{~nm}$.
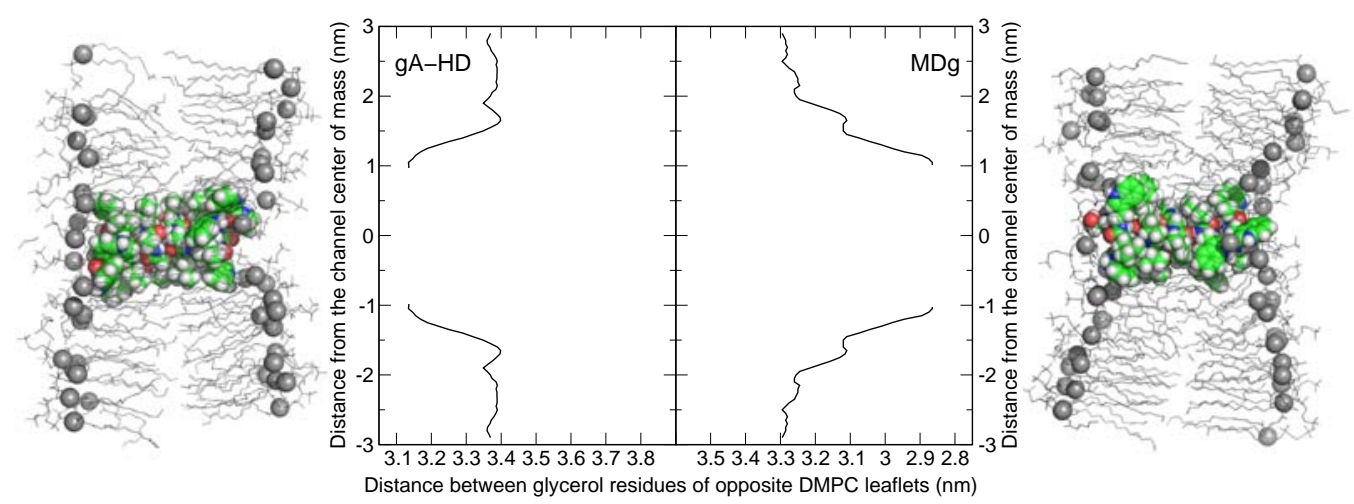

Figure 3.5.: Membrane thickness as function of the distance from the embedded peptide. The thickness is here defined by the average distance between the central carbon of the lipid glycerol moiety of opposing DMPC leaflets, selected from a circular segment of $0.05 \mathrm{~nm}$ centered at a distance $d$ from the peptide. The pictures on the side illustrate the effect of the peptides ( $g A-H D$ on the left, MDg on the right) on the DMPC bilayer. The central carbon of the glycerol residue is highlighted as a gray ball.

The radially averaged thickness as function of the distance to the center of mass of the peptidic channels is displayed in Fig. 3.5. Both peptides show remarkable effect on the surrounding lipidic environment. Presumably due to its smaller length, which sets the positions of the tryptophan groups deeper in the membrane, the local bending of the membrane around MDg is more pronounced than around gA-HD. For the MDg, a clear local thinning of the membrane occurs around the peptides. At further distances, beyond $2 \mathrm{~nm}$, the membrane thickens. The combination of the thinning and thickening of the membrane results in an average membrane thickness similar to the one of pure DMPC, as seen in Fig. 3.4 panel a. The gA-HD peptide induces a smaller curvature to its surrounding lipids, but the membrane is on average thicker than in pure DMPC bilayer: the match between hydrophobic and amphiphilic regions of the gA-HD - DMPC interface causes a global thickening of the DMPC bilayer normal to the membrane surface, that is propagated through the membrane. Due to the rela- 
tively small patch of lipids, or the high effective concentration of peptides, the membrane thickness does not decay to the value of pure DMPC at the largest distance from the embedded peptides.

The angle between the main axes of the peptides and the bilayer normal (illustrated in Fig. 3.6 panel b) is displayed as function of the simulation time in Fig. 3.6 panel a. Both gA-HD and MDg are found to be tilted with respect to the bilayer normal, the shorter channel being more influenced: the averaged angle for $\mathrm{gA}-\mathrm{HD}$ is $14.3^{\circ} \pm 0.1^{\circ}$, and $20.4^{\circ} \pm 0.1^{\circ}$ for $\mathrm{MDg}$.
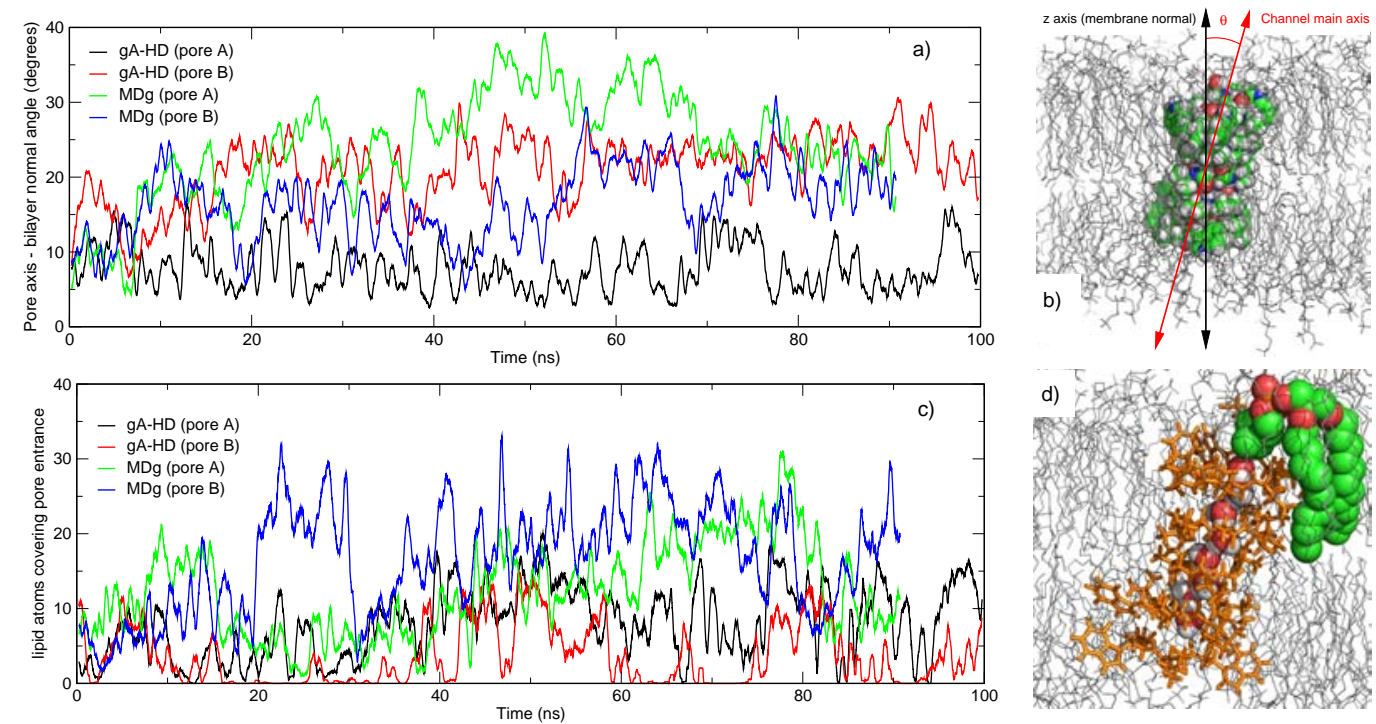

Figure 3.6.: a) Angle between the pore main axis and the bilayer normal (illustrated in panel b) as a function of the simulation time for the four studied channels. c) Time dependence of the number of lipid atoms covering the entrance of the peptidic channels. d) Snapshots of gA-HD (orange sticks) simulations in a DMPC bilayer (gray) showing the presence of a lipid molecule (displayed as balls) in the channel entrance. The head group of the lipid molecules disturbs the permeation of water molecules (white and red balls, only the water molecules in the channel are shown) For clarity, a running average using a $500 \mathrm{ps}$ time window was performed on the data for a) and c).

Due to the channel tilt and the membrane curvature around the channel, both the gA-HD and MDg channel entrances are disturbed by the lipid head groups. This interference causes a reduction of the available area for the water molecules to enter the channel. A snapshot of a representative configuration where the lipid head groups block the channel mouth is displayed in Fig. 3.6 panel d. To quantify this effect, we computed the number of lipid atoms lying above the channel entrance as function of the simulation time. We restricted 
the analysis to those lipid atoms that are within a cylinder with radius equal to the pore radius, defined by the peptidic backbone. To account for the channel tilting, the cylinder was aligned along the main axis of the channel. The time dependence of the number of lipid atoms covering the pore is displayed in Fig. 3.6 panel a. The mean value for $\mathrm{gA}-\mathrm{HD}$ is $5.89 \pm 0.1$ atoms/channel, and $14.56 \pm 0.2$ atoms/channel for MDg. The disturbances of the channel entrance are therefore in agreement with the induced curvature in the lipid bilayer and the average tiltangle: larger curvatures and tilt-angles allow more lipid head groups to approach the channel entrance.

Permeability coefficients Table 3.1 shows the water permeability coefficients, their ratio minus one (see equation 2.25) and water occupancy results for gA-HD and MDg. For comparison, experimental values for both gramicidin derivatives are also presented.

\begin{tabular}{cccccc} 
& & $p_{f}\left(10^{-14} \mathrm{~cm}^{3} / \mathrm{s}\right)$ & $p_{d}\left(10^{-14} \mathrm{~cm}^{3} / s\right)$ & $p_{f} / p_{d}-1$ & Pore occ. \\
\hline gA-HD & Pore A & $2.85 \pm 0.4$ & $0.39 \pm 0.1$ & $6.3 \pm 2.1$ & $6.7 \pm 0.1$ \\
& Pore B & $2.62 \pm 0.3$ & $0.28 \pm 0.05$ & $8.3 \pm 1.9$ & $7.1 \pm 0.1$ \\
& Exp. [144] & 1.6 & - & - & - \\
\hline \hline \multirow{2}{*}{ MDg } & Pore A & $4.10 \pm 0.4$ & $0.59 \pm 0.1$ & $5.9 \pm 1.35$ & $6.0 \pm 0.1$ \\
& Pore B & $4.20 \pm 0.5$ & $0.55 \pm 0.1$ & $6.3 \pm 1.65$ & $6.1 \pm 0.1$ \\
& Exp. [64] & 5.6 & - & - & -
\end{tabular}

Table 3.1.: Permeability coefficients, ratio of permeability coefficients and pore occupancy for the studied peptidic channels. For comparison, experimental values for the osmotic permeability are also listed.

The computed osmotic permeability coefficients are close to the experimental value, reproducing their tendency: MDg displays a faster permeability than gAHD. The ratio of osmotic permeability coefficients yields relatively good agreement with the occupancy via equation 2.25. Later on in this thesis, the validity of this equation will be discussed. To interpret the permeability results we have to take into account the pore characteristics and external factors that can affect the water permeation: accessibility, structural stability, length, radius, pore polarity, etc. The establishment of a clear structure-permeability relationship is problematic due to these multiple and coupled contributions. Although MDg tilts more frequently and it is on average more disturbed by the lipid head groups 
than gA-HD, the water permeability is nevertheless higher than gA-HD. Factors that could explain the faster transport of water molecules through MDg are its larger structural stability, the absence of pore blockage due to the missing formyl groups, a slightly larger radius ( $0.1 \mathrm{~nm}$ wider that $\mathrm{gA}-\mathrm{HD})$ and its shorter length (there is one water molecule less fitting in the pore than in gA-HD).

As we have seen, several factors are presumably involved in determining the permeability properties of a peptidic channel. A gradual approach is therefore required to understand the effects of channel architecture and permeability properties. In the rest of this thesis, we will therefore systematically investigate the individual effect of the channel length, the radius and the pore polarity on the water permeability of narrow pores.

\subsection{Conclusions and outlook}

Our simulations showed that gA-HD and the linked derivative MDg, both in a $\beta$-helix conformation, are stable in a DMPC bilayer. The capping groups of gA$\mathrm{HD}$, ethanolamine and formyl, were found to deviate the most from their initial configuration, partially blocking the channel. MDg shows a similar behavior in its $\mathrm{C}$ terminus, but the presence of the succinyl linker avoids the pore blockage due to the formyl group. Both channels affect the structure of the surrounding lipid bilayer by adjusting the bilayer thickness to best match the hydrophobic length of the channel and the amphiphilic tryptophan positions. The peptide gA-HD was found to slightly increase the membrane thickness, with a slight deformation of the surrounding lipids. The shorter MDg showed a much more pronounced bending of the neighboring DMPC bilayer. The combination of the channel tilt with respect to the bilayer normal and the mismatch between the overall membrane thickness (including the polar head groups) results in lipid head groups diffusing over the channel entry and interfering with water permeability.

The osmotic and diffusive water permeabilities were found to be in reasonable agreement with the experimental values, MDg permeates water molecules at faster rates than gA-HD. A general statement about the structure-permeability relationship solely based on the presented results is not possible due to the multiple phenomena affecting the water mobility (capping groups, membrane influence, pore length, pore radius, structural stability, etc), and therefore systematic 
studies where only one property is probed at a time are required.

However, the observation that lipid polar head groups disturb, and even impede, the permeation of water molecules through the pore can be used to design more efficient water channels. In order to enhance the water permeability a solution could involve protecting the channel entrance from the lipid head groups, while keeping the channel anchored perpendicular to the membrane plane.

To protect the channel entrance from the neighboring lipid head groups, a promising option is the incorporation of lipid-like residues directly attached to the gramicidin peptide at the pore mouth, coating the channel outer surface. By placing the lipid-like residues around the channel, the first layer of lipid molecules from the bilayer would effectively be positioned further away from the channel, consequently reducing the chances that a lipid head group blocks the channel. Furthermore, a favorable matching between the channel and the bilayer could be introduced by selecting different lengths of the hydrophobic attached to the peptide tails.

Since the tryptophan residues close to the pore mouth are important for anchoring of the peptide in the membrane [45], the leucine residues are, in principle, the best candidate for being substituted by a lipid-like residue.

Other factors that were found to disturb the permeation pathway are the formyl and ethanolamine capping groups. A linked gramicidin derivative, such as midigramicidin, does not suffer from interruptions in the water permeation due to the formyl groups, and additionally has a much longer channel life-time [129]. To avoid the intrusion of the ethanolamine capping group, a third coating lipidlike residue could be attached to the terminal alcohol, thus keeping the capping group away from the channel entrance. Studies along this directions are being currently carried out.

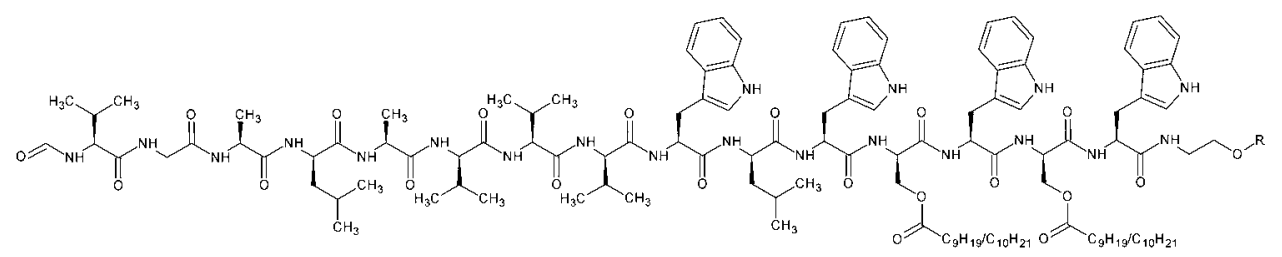

Figure 3.7.: A suggested modification of the gramicidin A monomer to improve the membrane match and reduce the intrusion of the lipid head groups in the channel entrance. The last two $D$-leucines were exchanged by an ester with a sufficiently long alkyl chain. The ethanolamine capping group can also be functionalized to include an alkyl chain. 
3. Gramicidin channels: a starting point 


\section{Influence of the pore length and polarity on water permeability}

\section{Summary}

We investigated the structural and energetic determinants underlying water permeation through peptipic nanopores, motivated by recent experimental findings that indicate that water mobility in single file water channels displays non-linear length dependence, and the results from the previous chapter. To address the molecular mechanism determining the observed length dependence, we studied water permeability in a series of designed gramicidin-like channels of different length using atomistic molecular dynamics simulations. We found that within the studied range of length the water permeability is independent of pore length. This result is at variance with textbook models, where the relationship is assumed to be linear. Energetic analysis shows that loss of solvation rather than specific water binding sites in the pore form the main energetic barrier for water permeation, consistent with our dynamics results. For this situation, we propose a modified expression for the osmotic permeability that fully takes into account water motion collectivity and does not depend on the pore length. Different schematic barrier profiles are discussed that explain both experimental and computational interpretations, and we propose a set of experiments aimed at validation of the presented results. Implications of the results for the design of peptidic channels with desired permeation characteristics are discussed.

As an extension, a subset of the designed gramicidin-like channels were modified to check for the effect of pore polarity on the length dependence. Pore occupancies and permeation coefficients are found to strongly depend on the polarity. The occupancy dependence on pore polarity is sigmoidal, and can be modeled by an absorption equilibrium. The permeability coefficients have a bell 
shaped dependence on the pore polarity, directly related to the behavior of water occupancies. Although several permeation mechanism operate at different pore polarities, within a given pore polarity all the channels show the same water mobility. The diffusive permeation coefficient shows a greater length dependence at intermediate polarities, i.e. where the water mobility is the highest. The ratio of osmotic and diffusive permeability coefficients was found to be linearly proportional to the average occupancy for any pore polarity.

\subsection{Invariance of water mobility in single file pores as function of length dependence}

\subsubsection{Introduction}

Recent experimental results [64] indicated that single file water channels show an exponential dependence of water mobility on the file length: reduction of pore occupancy by one water molecule drastically enhanced water mobility. Using gramicidin derivatives of different pore length, an exponential increase on water mobility was reported as the channel shortens. The studied channels were gramicidin A (30 amino acids), the cross-linked midigramicidin presented in the previous chapter (26 amino acids), and a very short symmetric cross-linked minigramicidin (22 aminoacids). The reported exponential dependence is at variance with the classic result from Finkelstein [5, 123], which assumes a linear relationship.

On the basis of these experimental findings and the long accepted linear prediction, we decided to investigate the microscopic details of the connection between water mobility and pore length. Taking advantage of computer modeling, we carefully designed a series of peptidic pores with directly comparable structural features embedded in an artificial membrane of controlled width, and characterized them using extensive atomistic molecular dynamics simulations. In doing so, we restricted the structural variations among compared channels solely to the length, making sure that secondary effects, like potential distortions due to sequence difference, membrane thickness and capping groups did not bias the results.

This study is discussed in the light of osmotic permeability $\left(p_{f}\right)$ and diffu- 
sion permeabilities $\left(p_{d}\right)$ as means of quantifying water mobility inside single file pores [123]. As explained in Sec. 2.2, the $p_{f}$ is related to the intrinsic water flux inside the pore $[61,96]$, while $p_{d}$ represents a net water transport between the compartments linked by the pore. Water mobility is therefore clearly related to the osmotic permeability coefficient, and the latter in return is related with $p_{d}$ via the occupation number [123]. To be able to elucidate structural determinants, we evaluated water free energy profiles and interaction profiles along the water pathway.

The length dependence of instantaneous water flux in the length range investigated $(1.4-2.5 \mathrm{~nm})$ is discussed, and we propose different underlying energetic schemes with the different observed behaviors, both experimental and computational. Finally, we tested the robustness of the presented results by exploring the effect of the backbone polarity on the length dependence.

\subsubsection{Theory and Methods}

Design and setup For the design of model pores to test the relationship between water flux and length it is crucial to separate the effects arising from other sources, such as linking groups with different conformations, channel symmetry, width of the lipid bilayer, etc. We regularized a set of D,L-polyalanine peptides with a typical $\beta$-helix gramicidin A folding motif, embedded in a membrane slab of adjustable thickness to avoid side effects due to non-matching lipid membranes.
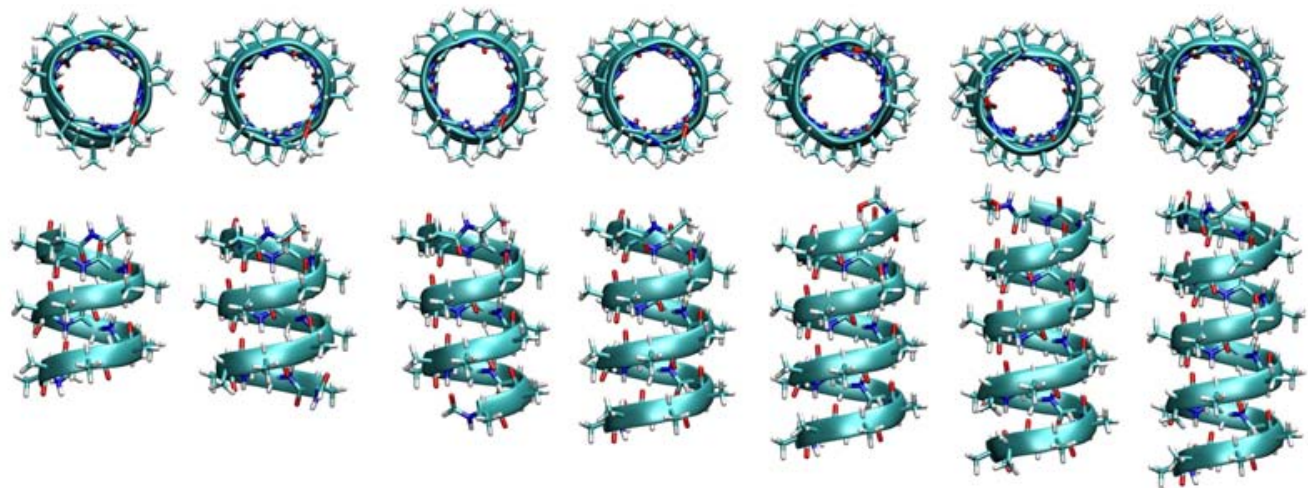

Figure 4.1.: Top and side view of the seven modeled polyalanine pores of increasing number of residues used for the study. From left to right, p-ala15 to p-ala27

Seven single file water pores have been prepared using one monomer of grami- 
cidin A in head-to-head helical dimer (HD) conformation as a template (Fig. 4.1). The template was constructed by averaging the available NMR structures (PDB codes 1GRM, 1JNO, 1MAG, 1NRM, 1NRU [131, 145, 146]) and setting all hydrogen bond between helix planes to the mean value to remove irregularities. All the residues were mutated to alanine to guarantee a set of directly comparable peptides, while keeping the capping groups formyl and ethanolamine at the $\mathrm{N}$ and $\mathrm{C}$ termini respectively. Due to the alternating $\mathrm{D}, \mathrm{L}$ amino acid sequence, the side chains point outwards from the pore lumen and do not affect the hydrophilic environment inside the pore caused by the amino acid backbone. The role of the side chains is to keep the structure stable and anchor the pore within the membrane, as shown experimentally in $[45,138,147,148]$ and by computer simulations in the previous chapter. The integrity of the folded structure was ensured by softly restraining the dynamics of the backbone and of the capping group atoms to their respective starting conformation using harmonic restraint forces of $500 \mathrm{~kJ} / \mathrm{mol} / \mathrm{nm}^{2}$.

The shortest unit, p-ala15, is then a mutated version of a gramicidin A monomer in HD conformation. We generated longer pores by increasing the number of amino acids in the sequence of the previous template in steps of two. Up to p-ala21, residues were added by extending the template from the $\mathrm{C}$ terminus. To obtain longer pores (p-ala23 to p-ala27), p-ala21 was additionally extended towards the $\mathrm{N}$ terminus. After energy minimization with restraints on the peptide main chain, the structures were inserted in a membrane. Using this procedure, propagation of inhomogeneities in building the tree dimensional arrangement is minimized, since all channels are directly based on the same template.

The disturbances of the pore caused by the membrane can be quite drastic, and affect pores of varying length to different extents, as we saw in the previous chapter. For a controlled environment, it is necessary to adjust the membrane according to the pore it accommodates. To do so, we devised an artificial membrane of octane molecules. This membrane offers the possibility to modulate its thickness via molecular dynamics pressure coupling, adjusting itself to the height of the inserted pore. The simulation systems were set up as follows. First, a simulation box filled with water was generated around the peptide. Then, a slab of water molecules was removed to accommodate the membrane. Next, octane molecules were added to the empty compartment. A short ( 150 ps) molecular 
dynamics equilibration was performed using pressure coupling and keeping the peptide fixed by means of strong position restraints $\left(5000 \mathrm{~kJ} / \mathrm{mol} / \mathrm{nm}^{2}\right.$ harmonic force constant to all atoms), finally obtaining a constant density and desired width for the octane slab. To keep the membrane stable over the course of simulations, we performed all molecular dynamics production runs using constant volume.

Interactions between all atoms in octane and peptides were described by means of the OPLS all-atom force field [78, 79], and water molecules were described by the TIP4P model [80]. A set of control simulations was carried out using the SPC water model [149]. All simulations were performed using the GROMACS simulation software $[134,135]$. Electrostatic interactions were calculated with the particle mesh Ewald method [93, 136]. Short-range repulsive and attractive dispersion interactions were simultaneously described by a Lennard-Jones potential, using a cut off length of $1.0 \mathrm{~nm}$. The Settle [83] algorithm was used to constrain bond lengths and angles of water molecules, and LINCS [82] was used for all other bonds and angles, allowing a time step of $2 \mathrm{fs}$. The temperature in the simulations was kept constant by weakly coupling the peptide, octane and water molecules to an external heat bath at $300 \mathrm{~K}$ [95]. When constant pressure simulations were required, a weak coupling to a pressure bath of $1 \mathrm{~atm}$ was used.

All simulations were run for $100 \mathrm{~ns}$. The simulations with TIP4P water model were repeated twice, using different starting velocities to enhance the statistics. The total simulation time amounted to $2.1 \mu \mathrm{s}$.

Single file water transport coefficients To describe the mobility of water molecules inside single file water pores we used the osmotic permeation coefficient $p_{f}$ and the diffusion permeation coefficient $p_{d}$, previously introduced in Sec. 2.2. Here we discuss which methodologies we chose to compute the permeability coefficients for this study, the text book connection between them and the length of the channel, and the water occupancy.

Since water motion in this channels is highly correlated, and the pores remain filled throughout the simulations, the $p_{f}$ was obtained by means of $p_{f}=\frac{1}{2} \Phi_{0} \nu_{w}$, where the intrinsic flux $\Phi_{0}{ }^{1}$ is computed as the rate of bidirectional collective jumps of the water column about a water-water distance (taken as $0.275 \mathrm{~nm}$ ).

\footnotetext{
${ }^{1}$ Previously we defined the single channel osmotic permeability as $p_{f}=k_{0} v_{w}$ (pg. 21). Therfore the intrinsic flux corresponds to $\Phi=2 k_{0}$.
} 
The textbook analytical expression,

$$
p_{f}=\frac{\pi r^{2} D_{w}^{0}}{L}
$$

as collected from [5], shows a linear relationship between the osmotic permeability coefficient and the pore length $L$ via $D_{w}^{0}$, the average diffusivity a water molecule would have it would be alone it the pore. $r$ is the the pore radius. $D_{w}^{0}$ is assumed to be independent of L. The arguments used for the derivations are grounded on the thermodynamic work driven by the osmotic pressure and the frictional force opposing the water transport. Since every molecule of the water column was assumed to experience the same additive frictional force, the velocity of the column was suggested to decrease linearly with L. It is important to note that the assumptions that lead to equation 4.1 implies that the pore is fully occupied with non-interacting water molecules, so that the collectivity of water motion is not taken into account. In this work we will study the validity of this approach.

The diffusion permeation coefficient $p_{d}$ describes the equilibrium flux between two compartments of equal concentration. In this regime, a permeation event consists of the transport of a given water molecule from one side of the channel to the other. Similar to $p_{f}$, the diffusion permeation coefficient is computed as $p_{d}=\frac{1}{2} \Phi_{c} \nu_{w}$, where $\Phi_{c}$ is the number of bidirectional complete water translocations $^{2}$. Because complete permeation events are rare compared to water-water distance movements, we make use of the symmetry of the pore and count the number of water molecules crossing half of the pore to increase the statistical accuracy. Considering that it is four times more probable to travel half a distance than completing a full translocation passage, the number of complete permeations is one fourth of the half permeation events. We validated our approach by comparing the results obtained from both counting methods, with excellent agreement (data not shown).

Within the single-file framework, the ratio between the two permeation coefficients in a fully occupied pore is assumed to be equal to $n+1$ or $n[5,96,101]$, where $n$ is the number of single-file water molecules that fit into the channel. Therefore, a consistency check for the obtained permeabilities is to test if this equality is fulfilled. The ratio of permeability coefficients can be directly com-

\footnotetext{
${ }^{2}$ With the previous definition of $p_{d}$ (pg. 19), $\Phi_{c}=2 k_{d}$
} 
pared to the pore occupancy estimated from the simulations. Direct calculation of the pore occupancy was done by averaging the number of water molecules within the pore over the simulation time. Cylindrical pore boundaries were used, defined by the radial cut-off imposed by the pore backbone and the averaged position of the first and last peptide's residue at every time step.

Thermodynamic properties Free energy profiles $F(z)$ of water molecules moving along the pore axis (defined as the $z$ axis) have been calculated using two different approaches. Since the production simulations were run at constant volume, we can directly access the Helmholtz free energy $F(z)$ via the water number density $\rho(z)$ as $F(z)=-k_{B} T \ln \rho(z)$, with $k_{B}$ the Boltzmann's constant and $T$ the temperature, see Sec. 2.3.1. The number density was evaluated in $0.01 \mathrm{~nm}$ bins over the simulation time. This approach yields the total contribution of the whole system to each single water molecule as it permeates the pore. To achieve a better understanding of the determinants affecting the transported molecules, we also calculated the potential energy surface along the pore axis by integration of the average forces acting on the single water molecule $F(z)=\int_{z_{1},\left\langle f_{z}\left(z_{1}\right)\right\rangle=0}^{z}\left\langle f_{z}\left(z^{\prime}\right)\right\rangle d z^{\prime}$, with $z_{1}$ the reference position where the average force vanishes. Hence, we use the term of Potential of Mean Force (PMF) for these profiles. This allows us to qualitatively assess contributions of the peptide and those imposed by the rest of water molecules by independently integrating the averaged forces involved in their interactions. Note that profiles of individual components do not necessarily reflect free energies, and can only be interpreted qualitatively.

It is worth stressing here that the picture obtained from such a free energy profile corresponds to the combined effects of water collectivity and interactions with the pore as well as the rest of the water. We validated the PMF procedure by comparing the profiles with the number-density result (see figure 4.5).

Monte Carlo simulation on PMF Water mobility in single-file pores is highly correlated. Therefore, water permeation can be treated as a collective motion of the single-file along the pore (figure 4.2).

To characterize the relationship between the behavior and energetics of individual water molecules, we used Metropolis' Monte Carlo algorithm to move a chain of water molecules over a schematic potential energy surface. By con- 

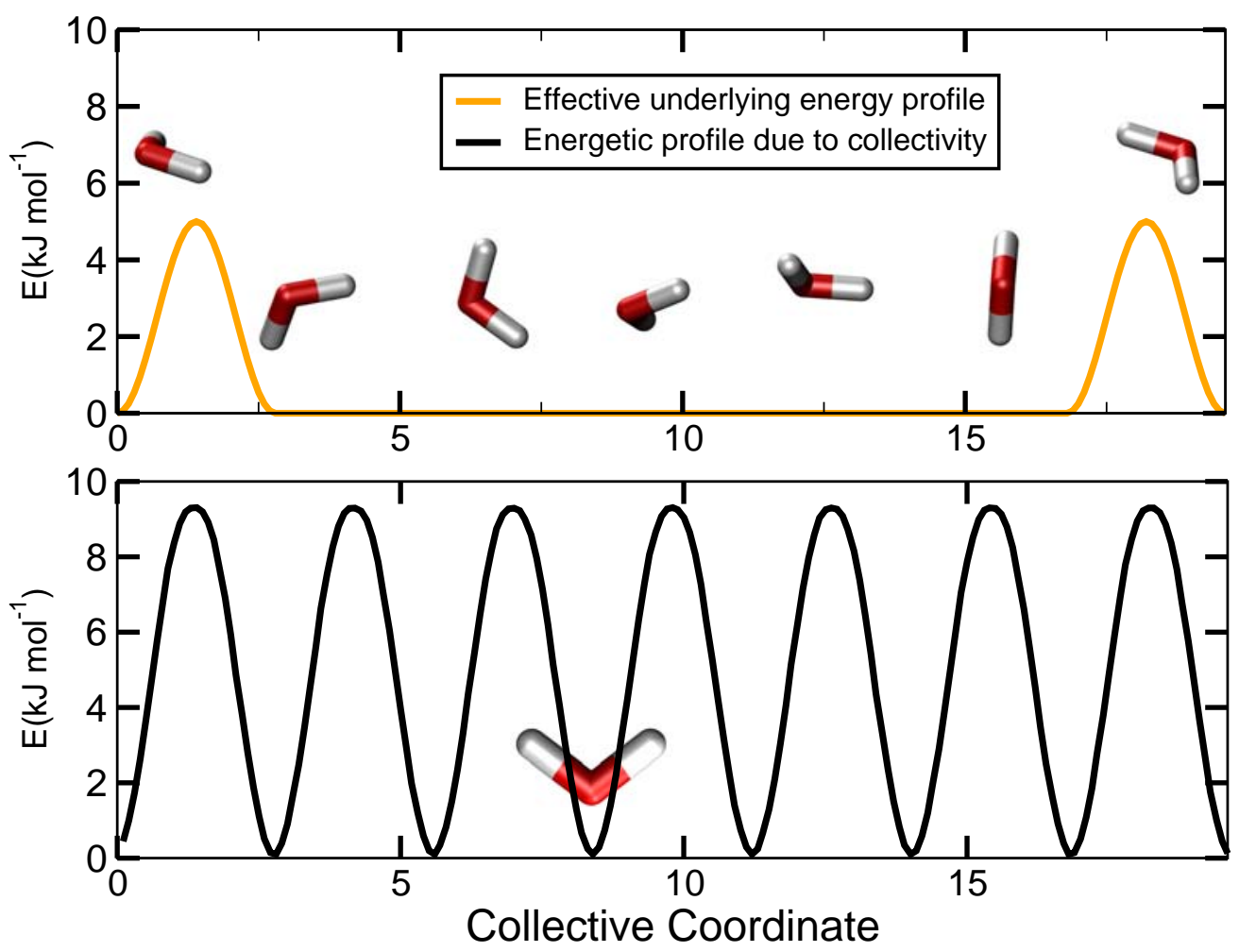

Figure 4.2.: Effect of water collectivity on the underlying potential for motion along a singlefile pore. The upper panel depicts a situation where two barriers are found at the entry/exit regions. The water molecules are sketched on top of the potential energy surface for illustration. Using the Monte Carlo method to sample the motion of a chain of water molecules, we can derive a free energy profile from the number density (lower panel). As a consequence of the imposed correlation between water positions, the emerging energetics seems to indicate the presence of local binding sites, whereas the true inner potential surface is actually flat.

sidering the chain of water molecules as rigid, we introduced collectivity to the system. The step size was fixed, and adjusted to produce $\sim 70 \%$ accepted moves for barrier heights comparable to those extracted from our simulations. In this framework, a " $p_{f}$ event" is counted every time the water chain has crossed a water-water distance, and a " $p_{d}$ event" is computed when a water molecule has traveled the whole channel length.

Although no true rates can be computed by this approach, it provides useful relative information of the transport coefficients. If the step size is kept constant for all channel lengths, a series of pores can be compared to obtain qualitative dynamic trends. This method is particularly useful to probe different underlying potentials by allowing to screen various energetic scenarios for their compatibil- 
ity with the MD results. It fully takes into account collectivity, and allows to decompose obtained free energy profiles into contributions due to channel-water interaction and water-water correlation.

\subsubsection{Results and discussion}

Figure 4.3 shows the cumulative count of water-water displacements of the water column inside the pore as function of the simulation time.

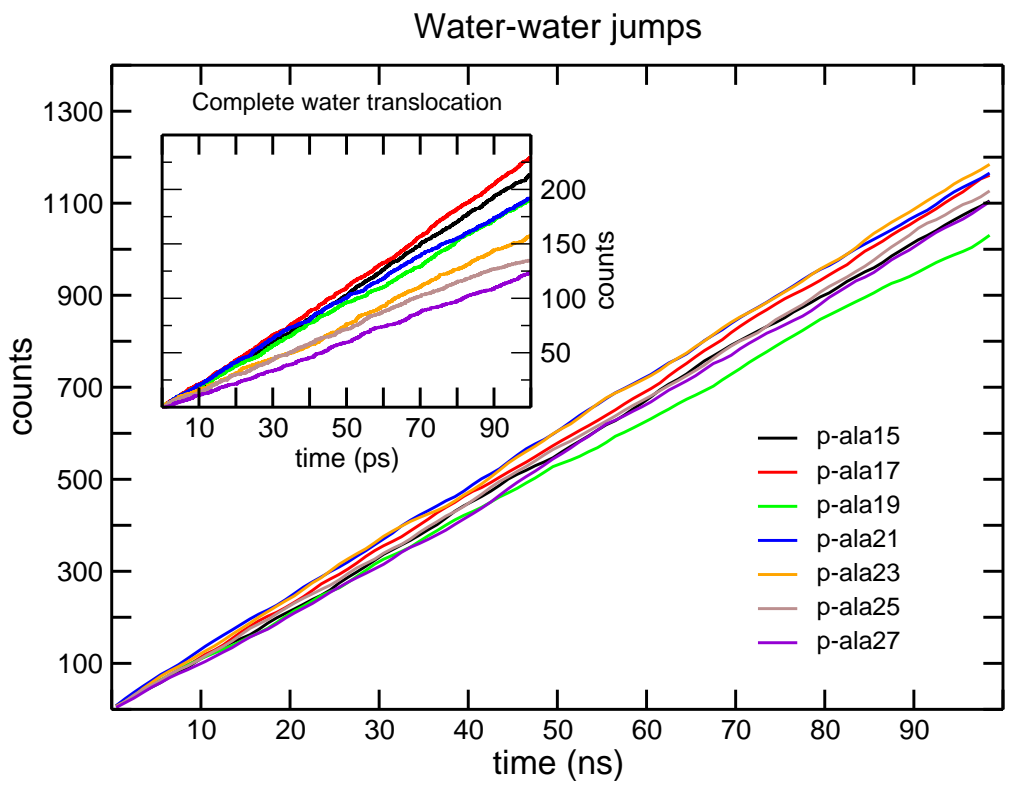

Figure 4.3:

Cumulative waterwater displacements, (taken as $0.275 \mathrm{~nm}$, main frame) and complete water translocation (after correcting for half permeation counting, inset) for all seven pores studied, averaged over two independent simulations.

The slope of the curve is directly proportional to $p_{f}$. In all seven cases, the water flux displays a steady converged behavior and shows no clear dependence on pore length. In contrast, the cumulative number of complete water translocations from one side of the pore to the other (inset Fig. 4.3) exhibits an overall decreasing slope with growing peptide length. The slope of the curve is in this case proportional to $p_{d}$.

Figure 4.4 presents the osmotic permeability coefficient (a) and the diffusion permeability coefficient (b) as a function of the number of amino acids in the sequence, averaged over two independent sets of simulations. The osmotic permeability coefficient $p_{f}$, which is related to the intrinsic water mobility in the channel, is found to be independent on the length, within the simulation accuracy. Very low sensitivity of water flux and pore length was previously [150] for the osmotic water flow through carbon nanotubes. 


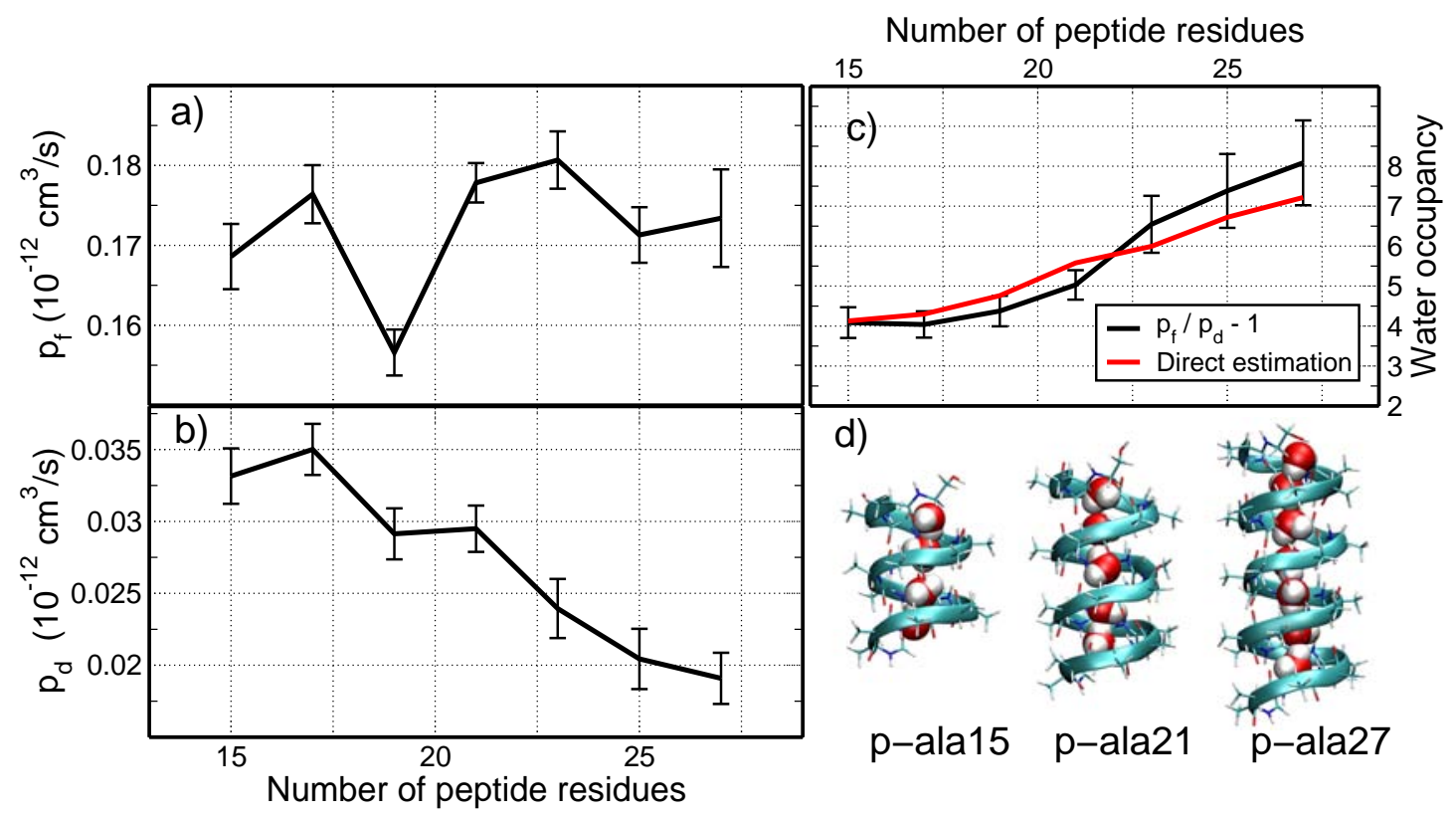

Figure 4.4.: Averaged (a) osmotic permeability coefficient $\left(p_{f}\right)$ and (b) diffusive permeability coefficient $\left(p_{d}\right)$ from two independent simulations as function of the number of residues forming the channel. Panel (c) displays the relationship between residue number and water occupancy as computed via $n=p_{f} / p_{d}-1$ (black) and estimated from the simulation (red). (d) illustrates water occupancy for p-ala15, p-ala21 and p-ala27.

As expected, the diffusion permeability coefficient displays an inverse relationship with the length. In panel (c) we represent the water occupancy for each channel as a function of the number of residues. This can be directly estimated from the simulations (red) or computed via the relationship $p_{f} / p_{d}=\mathrm{N}+1$ (black). Remarkably good agreement with both independent measurements is found. This is a strong indication that the assumptions of memoryless water jumps and water motion collectivity are indeed fulfilled.

The calculation of the free energy for permeating water (figure 4.5) reveals a main access barrier of $\sim 3.6 \pm 0.2 k_{B} \mathrm{~T}$ for both pore entrances. Compared to this barrier, the energy required to move from one binding site to the next within the pore is small, in the order of $k_{B} \mathrm{~T}$. Similar behavior was also observed in carbon nanotubes [150]. Results obtained via force integration or water density are nearly identical, as shown in the comparison panel for p-ala25 in Fig. 4.5

Decomposition of free energy in water-water and water-protein interactions shows that both profiles are generally complementary, and loss of energy due to desolvation is partially counteracted by the attractive interaction with the 

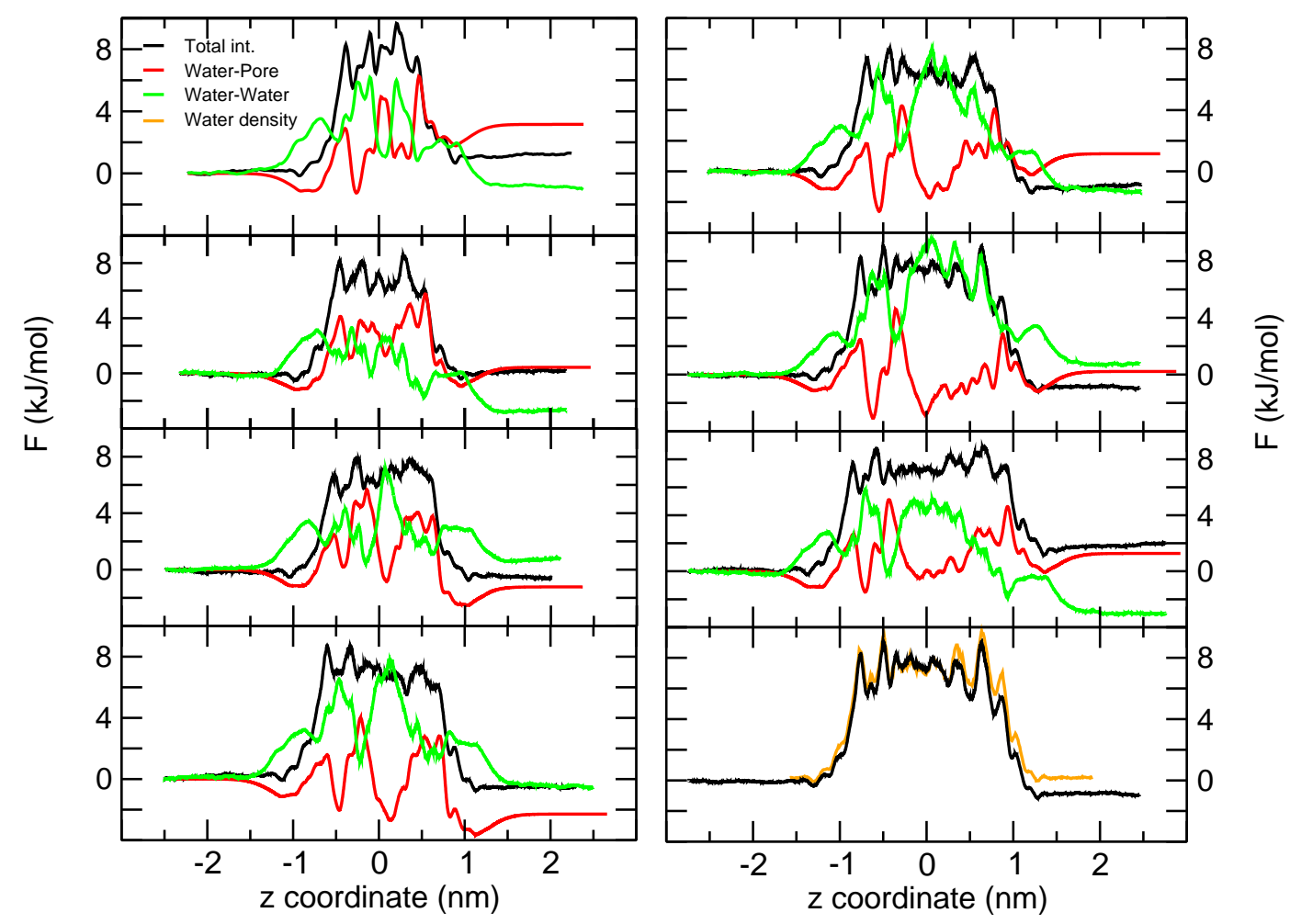

Figure 4.5.: Free energy profiles and their decomposition for the series of peptidic pores as obtained from force integration (see text). Total interaction (black), water-pore interaction (red) and water-water interaction (green) are plotted against the main pore axis. The panel on the lower right shows a comparison of free energy profiles as computed via water number density (orange) with force integration (black) for p-ala25.

peptide. Altogether, barriers due to solvent interaction are predominant. As the peptide grows in length, the fragment of the curve describing the inner part of the channel becomes flatter. Presumably the smoothening in the central channel region indicates that there is room for a non-integer number of water molecules in the channel. This would result in a deviation from the full correlation depicted in an idealized way in Fig. 4.2.

In a situation in which the barriers near the channel entry form the ratelimiting step for water permeation, the picture derived from decomposition of the interactions is consistent with the dynamic result that the osmotic permeability coefficient is independent of the length of the single file.

Underlying energy profile To study the observed behavior in terms of the underlying free energy profile, we designed a number of schematic topologies for 
the potential of mean force. On these constructed profiles we characterized the behavior of the two transport coefficients, $p_{f}$ and $p_{d}$, as a function of the single file length via Monte Carlo sampling. In the first scenario, each water position was separated from the next one by a constant height barrier (Fig. 4.6, two upper panels). The barrier height represents the "binding" free energy of water in each mean occupation site, and no extra activation energy is required to access the pore. Increasing the number of water positions means adding new barriers. Depending on on the barrier height, two different regimes can be discerned. If the binding free is below thermal energy $k_{B} \mathrm{~T}$ (Fig. 4.6, first from the top), the emerging picture corresponds to the textbook hypothesis of $p_{f} \sim 1 / n$ and therefore $p_{d} \sim 1 / n^{2}$.
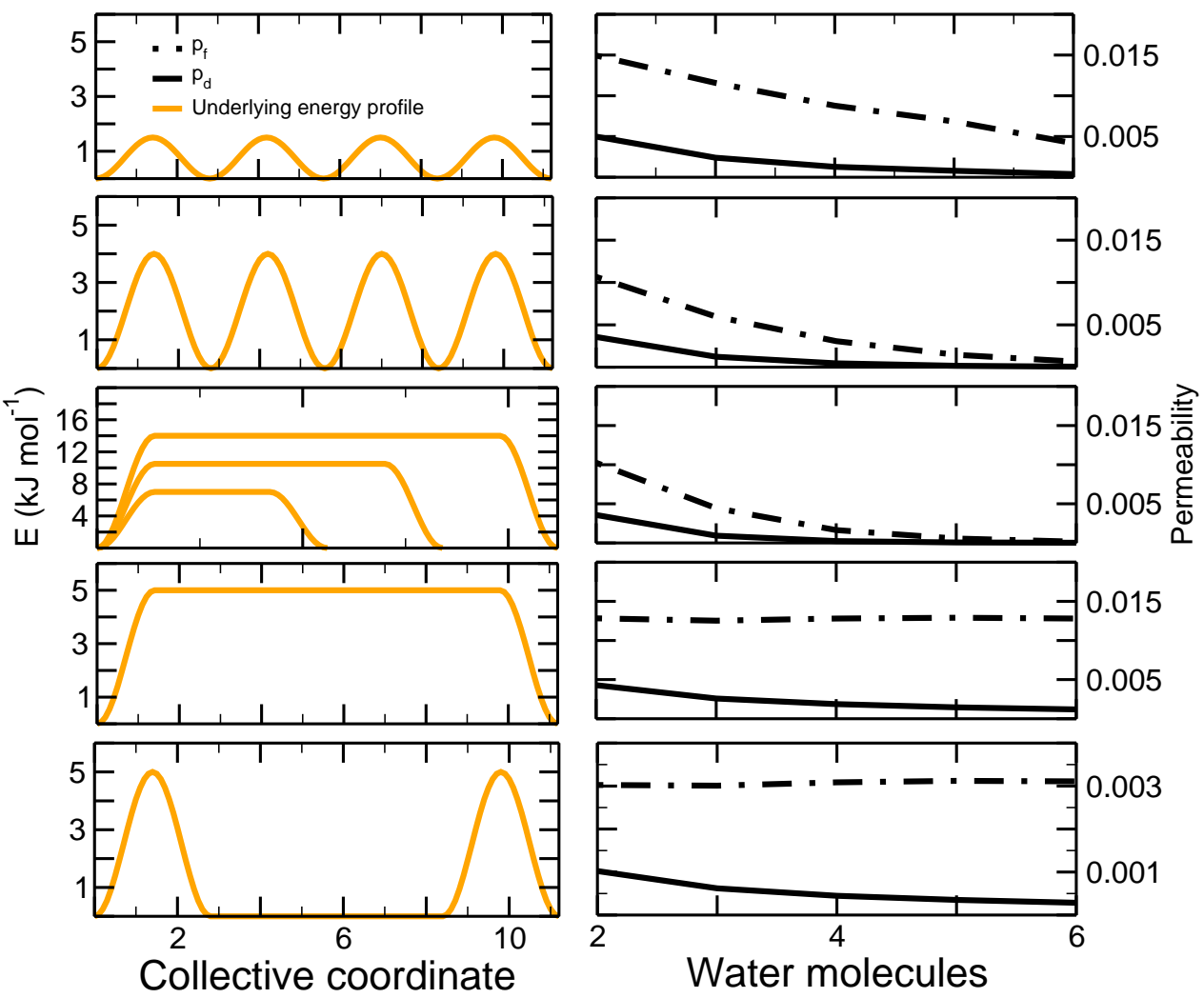

Figure 4.6.: PMF schemes for a chain of water molecules permeating a single file pore (left, gray) lead to a different length dependence (right), expressed in permeability coefficients $p_{f}$ (dashed dotted) and $p_{d}$ (black). Only (d) and (e) are consistent with the simulation results, suggesting that no binding sites for individual water molecules are present within the pore, and that the main barrier is independent of pore length. 
The result can be rationalized as follows: since the thermal energy of the system is above the binding free energy, the exponential dependence of the transition probability as function of the activation energy can be approximated linearly. Hence, the decay can be described as an inverse function of the activation energy, linearly dependent on the number of water molecules. Alternatively, if the activation energy for a water-water displacement is above the thermal energy (Fig. 4.6, second from the top), including more water molecules in the single file leads to an exponential dependence of both transport properties, similar to the experimental observations [64]. In the case of a single access barrier, such as in the last three profiles of Fig. 4.6, water mobility becomes dependent of the length of the single pore if the height of the barrier is variable. In such a situation, illustrated in Fig. 4.6 c, an exponential or linear dependence of water permeability is expected depending on the range of barrier heights, analogous to the situation in Fig. 4.6 b.

On the contrary, an access barrier with a fixed height displays water mobility independent of the single file length. $p_{d}$ shows a characteristic inverse dependence, simply explained by a linear relationship between step size and coordinate length. As long as there is an access barrier, relative energies between inside/outside do not lead to formal differences in the expected dynamics of a highly correlated water file along a flat inner potential. This situation is consistent with the simulation results, and in conjunction with the observed permeability behavior suggests that the motion of the water column is governed by two or more access barriers or a central barrier of constant height. This hypothesis is further supported by the drastically altered water permeability found in gramicidin derivatives with mutated termini, e.g. in desformyl gramicidin [39, 61], or modified residues at the channel entry as shown in desformyl gramicidin [39, 61], or modified residues at the channel entry, such as Lysin gramicidin (unpublished data).

In light of the obtained results, water permeability of gramicidin-like single-file peptidic water pores is length-independent. Hence, the simulation results suggest that for the design of new channels length is not a critical factor.

Corrections to equation 4.1 are mandatory to describe the observed behavior. A key assumption made in equation 4.1 is the independence of water molecules in the pore, i.e. interactions between water molecules are not taken into account. 
If we consider the collective nature of the single-file column, every water-water displacement contributes one water molecule to the observed osmotic flux. Using the same procedure as in [5] but identifying the water-water distance $d_{w w}$ as the distance the collective coordinate needs to travel to observe the net transport of one water molecule we arrive at

$$
p_{f}=\frac{\pi r^{2} D_{c}^{0}}{d_{w w}}
$$

where we use $D_{c}^{0}$ is the effective diffusivity of chain of water molecules and $r$ is the radius of the pore. Note that the obtained expression is indeed independent on the length of the pore or the number of water molecules.

The apparent discrepancy to the recent experimental findings [64] may be caused by simulation artifacts, like force field inaccuracies. In a set of control simulations where we used the simple point charge (SPC) water model instead of TIP4P, however, we saw qualitatively consistent results. Also, we can not exclude the possibility that the osmotic pressure caused by the osmotic gradient induces conformational changes in the channel. This unlikely effect is not not captured by the present approach to obtain osmotic permeabilities from equilibrium simulations.

Alternatively, the great experimental variance of single channel water permeabilities which cover three orders of magnitude [151] is most probably not due to length changes. Although the experimentally studied channels might have similar folding motifs, additional factors like unequal capping groups, differences in channel hydrophobicity, channel ability to form hydrogen bonds with permeating water molecules, membrane mismatch or cross-link effects could lead to changes of the access barriers, and thus of permeability. Especially, interactions with the membrane are expected to play a major role in determining water permeability. Simulation studies have identified a strong influence on water permeability by interactions of lipid head groups with the pore entrance $[61,124]$. It would therefore be interesting to systematically study effects of the surrounding membrane or to modify membrane-channel interactions in the entry regions. A promising approach to investigate water permeation, recently applied in the design of ion channels [152], is the possibility to funnel the permeating molecules into the channel by means of ether coronas or similar derivatives. This way one could also partially diminish the effect of membrane mismatch and disturbances due 
to lipid head groups.

Additional experiments will therefore be required to resolve this issue. Different essays on systems with varying bilayer width and constant gramicidin length would quantify the role of the external barriers on water permeability. Longer gramicidin like channels should be experimentally measured for the validation of the different predictions based on the obtained results.

\subsubsection{Summary and Conclusions}

In order to understand the main determinants of water permeation through single file peptidic pores the influence of the channel length has been addressed. We designed a series of regularized D,L polyalanine peptides in a $\beta$-helix folding with increasing number of residues to isolate the effect of the channel length on the dynamics of water transport. The analysis of water mobility in terms of osmotic permeability $\left(p_{f}\right)$ reveals no dependence on the length of the pore. Accordingly, the diffusion permeability $\left(p_{d}\right)$ displays an inverse relationship with the length of the pore: at constant hopping rate, the longer the pore: at constant hopping rate, the longer the channel the more time is needed for a complete translocation. This result is at variance with the classical view of a linear length dependence $[5$, $123,153]$ and also with a recent experimental study that indicated an exponential length dependence [64]. Inconsistencies of the proposed linear relationship can be corrected by identifying the displacement of the water column by a water-water distance as the effective transport step. The resulting expression becomes pore length independent. To resolve the discrepancy with the exponential prediction, additional experiments are required.

The potential of mean force for a water moving along the channel main axis shows that the main barriers for water permeation are located at the channel entries. The main access barrier is $\sim 3,6 k_{B} \mathrm{~T}$, independent of the channel length. The water binding energies inside the pore are more than three times lower, around $1 k_{B} T$. Decomposition of free energy in water-water and water-peptide contributions identifies desolvation as the main origin of the access barrier.

Monte Carlo simulations on Potential of Mean Force allowed us to test different underlying energetic profiles and their consequences on the mobility depending on coordinate length. We identified three different behaviors for $p_{f}$ and $p_{d}$ depending the nature and position of the barriers. The simulation results are consistent with 
a situation where fixed height barriers are located in the channel entries: in this case no correlation between channel length and water mobility is found.

Our results suggest that especially modifications at the channel entry and exit, as well as controlled interactions of the channel with lipid head groups are critical determinants for water permeation. For the design of nanopores with desired permeation characteristics, this implies that channel hydrophobicity in the entry/exit region and compatibility to the surrounding membrane, rather than channel length, are the major determinants underlying water permeability. 


\subsection{Effect of channel's backbone polarity on the water permeability length dependence}

The experimentally observed exponential dependence of water mobility on the length of the single-file channel [64] was not reproduced by our atomistic simulations. As noticed earlier, other simulation studies already recognized the small effect of the length on water permeation [150]. Several factors that could lead to the experimental observations were discussed in the previous section. It is known that variatons in channel polarity lead to changes in the nature of water transport, ranging from immobilized water molecules [154] to burst-like behavior of the water column [31, 32]. So far, systematic computer simulations on the effect of pore polarity were carried out on artificial model pores consisting of methane-like pseudo-atoms [155]. Even though this study included a few dipoles to test the effect of increased hydrophilicity, it is unclear if these results can be extrapolated to peptidic environments. Because direct experimental observation of water motion inside peptidic channels is not feasible up to date, transport in such pores has to be studied indirectly, e. $g$. by macroscopic conductance measurements. Whether the change in transport mechanism can lead to the experimentally observed change in mobility remains an open question.

Here we explore the effect of the polarity of the peptidic channels on the water permeability for channels of different lengths. Our main focus is on the permeability coefficients as means of describing the mobility of water molecules in the channel, their connection with pore occupancies, and the nature of the single-file transport.

Design of model channels We used a subset of the previously introduced polyalanine channels embedded in an octane slab (see Sec. 4.1.2) to investigate the effect of backbone polarity on the nature of the water transport at different pore lengths. Because the computational effort needed to cover multiple pore polarities is more demanding than in the previous work, we restricted our study to p-ala19, p-ala23 and p-ala27. The selected channels span a length similar to that of minigramicidin-gramicidin channels, and the number of water molecules that can fit in their lumen differ by one between consecutively longer channels.

Gramicidin-like channels form pores by directing all the sidechains away from 
the channel, due to their characteristic alternation of D and L aminoacids. Therefore, a change of the channel sequence would not result in evident and homogeneous changes in polarity. Instead, to modulate the polarity, we directly altered the partial charges of the peptidic backbone by modifying the dipole moment of the peptidic carbonyl groups. To this end, the partial charge on the carbon atoms were varied from +0.3 e to +0.6 e in steps of 0.05 e, along with the corresponding opposite charge for the oxygen to guarantee the overall charge neutrality. Since the carbon-oxygen length is fixed by the constraint algorithm, the dipole moment of these carbonyl groups ranges from 1.72 to 3.46 D. The OPLS force-field uses values of +0.5 e and -0.5 e respectively for the carbonylic carbon and oxygen partial charges, which corresponds to a dipole moment of $2.88 \mathrm{D}$. Since the rest of the partial charges are not changed, the channel remains partially hydrophilic even at low carbonyl dipoles.

Figure 4.7: Top and side view of the channel p-ala23. The positions and alignment of the carbonyl groups is indicated by spheres, carbon atoms in green and oxygens in red.

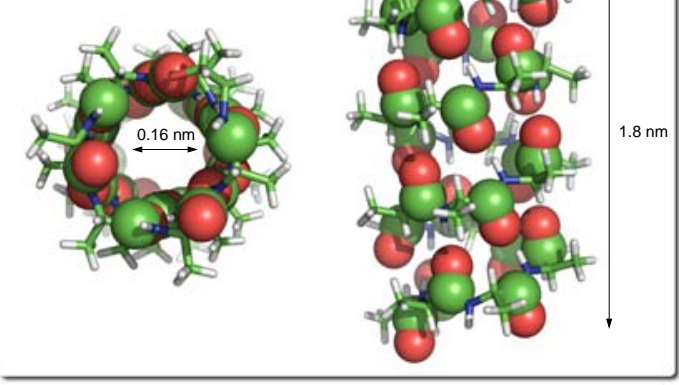

The set-up of the simulation systems was done as previously described. To obtain initial structures for the channels at different polarities, we started from previously equilibrated initial structures of the channels in their standard OPLS parameters, and we performed 2 ns molecular dynamic equilibration runs under the new polarity conditions. From these re-equilibrated set of structures, two sets of 100 and $150 \mathrm{~ns}$ molecular dynamics simulations with different initial velocities were carried out. The second set of simulations was more extended to ensure good sampling of the channels with low polarity. For each combination of charge distribution and channel length the simulations were done under the same thermodynamic conditions as in the previous study: weak coupling to an external bath at $300 \mathrm{~K}$, constant volume simulations, soft position restraints $\left(500 \mathrm{~kJ} / \mathrm{mol} / \mathrm{nm}^{2}\right.$ harmonic force constant) towards a reference structure, full long-range electrostatics (PME) and an integration step of 2 fs. 


\subsubsection{Results and discussion}

Pore occupancy as function of the backbone polarity The most immediate consequence of modulating the pore polarity is the drastic change in pore occupancy, denoted as $n$. Due to the reduction of stabilizing water-pore interactions, the free energy barrier for entering the pore increases. The chemical potential for a water molecule inside the pore, which is related to the probability $p$ that a particle can be inserted in the pore as $-k_{B} T \ln p$, increases with lower water-pore stabilizing interaction. Since the chemical potential of a water molecule in the bulk remains constant, the overall probability for a water molecule to enter the pore is reduced.

To render the measured occupancies comparable among pores of different length, we normalized all averaged pore occupancies $\langle n\rangle$ to the maximum occupancy $N_{\max }$. The normalized occupancy is then $\langle\theta\rangle=\langle n\rangle / N_{\max }$. Fig. 4.8 shows the normalized pore occupancies as function of the dipole moment of the carbonyl group of the peptidic backbone for the series of studied channels. The results were averaged over two independent trajectories for each backbone polarity and channel length.

The normalized pore occupancy is almost insensible to length of the pore, with small deviations at low channel polarities. The change in the curvature of the occupancy as function of backbone polarity occurs at the same pore polarity for all channels, an indication that the same mechanism operates for all pores studied. Note that the occupancy in the pore is not zero at zero dipole moment of the carbonyl groups. Formally, the appropriate independent variable should be the overall averaged energetic interaction between the pore and the water molecules. In the absence of a pore, implied by the absence of energetic interactions, the occupancy is necessarily zero. Nevertheless, we retain the present formulation for convenience.

To describe the observed behavior we closely followed the derivation in [32]. When we consider the pore and the water molecules in it as our thermodynamic system, the rest of the components in the simulation box act as external bath of constant chemical potential. Therefore, the appropriate thermodynamic ensemble to describe the varying water occupancy in the pore is the Grand Canonical, 


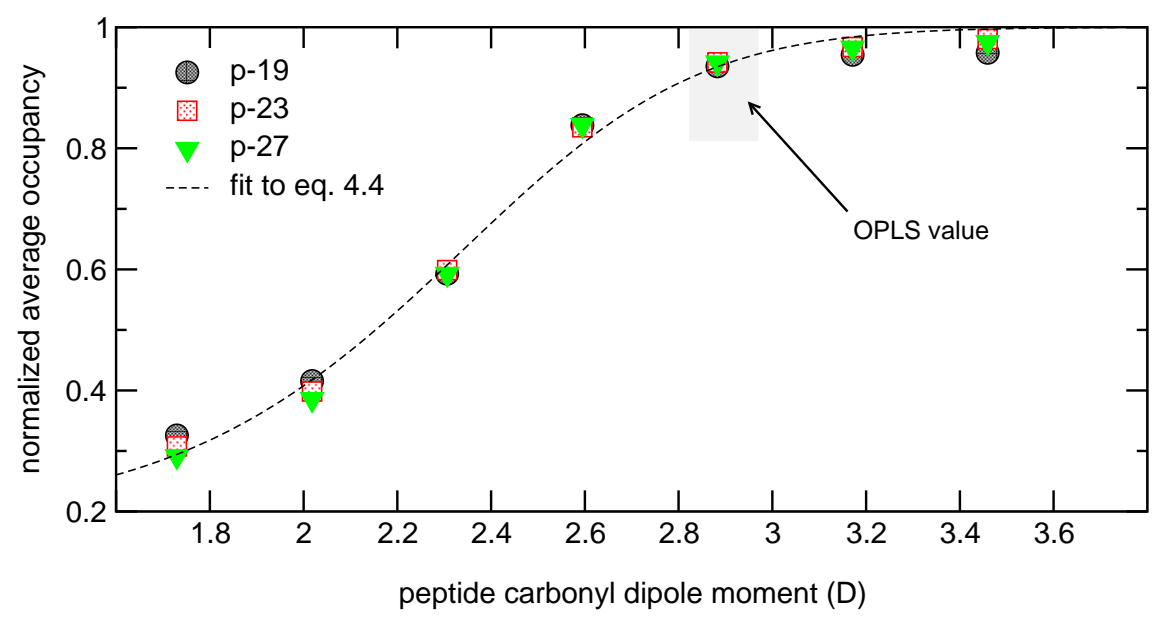

Figure 4.8.: Normalized average water occupancy for a series of polyalanine pores as function of the dipole moment of the peptide backbone carbonyl groups. The light gray area indicates the occupancy/dipole moment in the unaltered OPLS force-field. The dashed line indicates the fit to equation 4.4. A quadratic expression for the free energy was employed for the fit to the whole set of data points.

$\Omega(f, T, V)$, where $f$ is the fugacity, $T$ is the absolute temperature and $V$ the volume of the system. The ratio of unoccupied to occupied pore positions is proportional to the exponential of the free energy difference between a water molecule in the bulk and in the pore,

$$
\frac{1-\langle\theta\rangle}{\langle\theta\rangle}=e^{-\beta \Omega(f, T, V)} .
$$

A simple rearrangement leads us to the expectation value of the normalized average pore occupancy as function of the changes in free energy,

$$
\langle\theta\rangle=\frac{1}{1+e^{-\beta \Omega(f, T, V)}} .
$$

Equation 4.4 requires at least a quadratic expression for the free energy as function of the carbonyl dipole moment for the grand canonical potential to reproduce the occupancies obtained from the molecular dynamics simulations, implying that the relationship between the free energy and carbonyl group dipole is not linear. Due to the nature of dipole-dipole interactions between the water molecules and the carbonyl groups, the change in interaction energy is linear with the variation of backbone charges for a given dipole-dipole distance. However, the average distance between a water molecule and the pore is affected by the change 
of polarity, which also affects the energetic contribution besides the larger dipole moment of the carbonyl groups. Even more, the entropic contribution is also affected by the interaction energy, leading to further deviations from a linear relationship between the free energy and the dipole moment of the carbonyl groups.

Since a truly thermodynamic description requires an average over the whole phase space, we assumed that our system is ergodic and used a time averaged pore occupancies as the expectation value for the number of water molecules inside the pore in equilibrium conditions. This detail is important, since a given average occupancy does not imply that the pore is always filled with a fraction of the maximum available water occupancy. Rather, the pore might be almost fully occupied for a fraction of time during the time evolution of the system [32]. Likewise, it is in principle feasible that just a fraction of the pore positions are actually occupied at any given moment.

Visual inspection shows that the inner part of the pore is almost empty for low pore polarities, with small fluctuations at the entrance of the pore. Intermittent excursions of clusters of water molecules develop over time (see Fig. 4.9), and are virtually always connected to one of the two water compartments.

The average size of the cluster depends on the polarity of the pore (see Fig. 4.10), depends on the polarity of the pore (see Fig. 4.10), and correlates with the averaged occupancy. Eventually, the clusters grow enough to form a complete water column from one compartment to the other. Water molecules can jump from one protruding water column to the other more easily the closer they get. This behavior, described as liquid-vapor fluctuations [32], has been observed in hydrophobic pores as well.

Figure 4.10 shows the distribution of the number of water molecules inside the p-27 peptidic channel for different pore polarities. The distribution shifts towards larger clusters as the polarity of the pore increases. At intermediate polarity, the distribution shows a greater variance, illustrating that clusters of different sizes form and disrupt as the water molecules travel through the pore. As the pore occupancy saturates, the single-file column becomes the most stable structure. The OPLS charges lead to a water column that is almost intact, but a small decrease in the charge assigned to the carbonyl group leads to a substantial decrease in the stability of the complete water column. Since gramicidin 

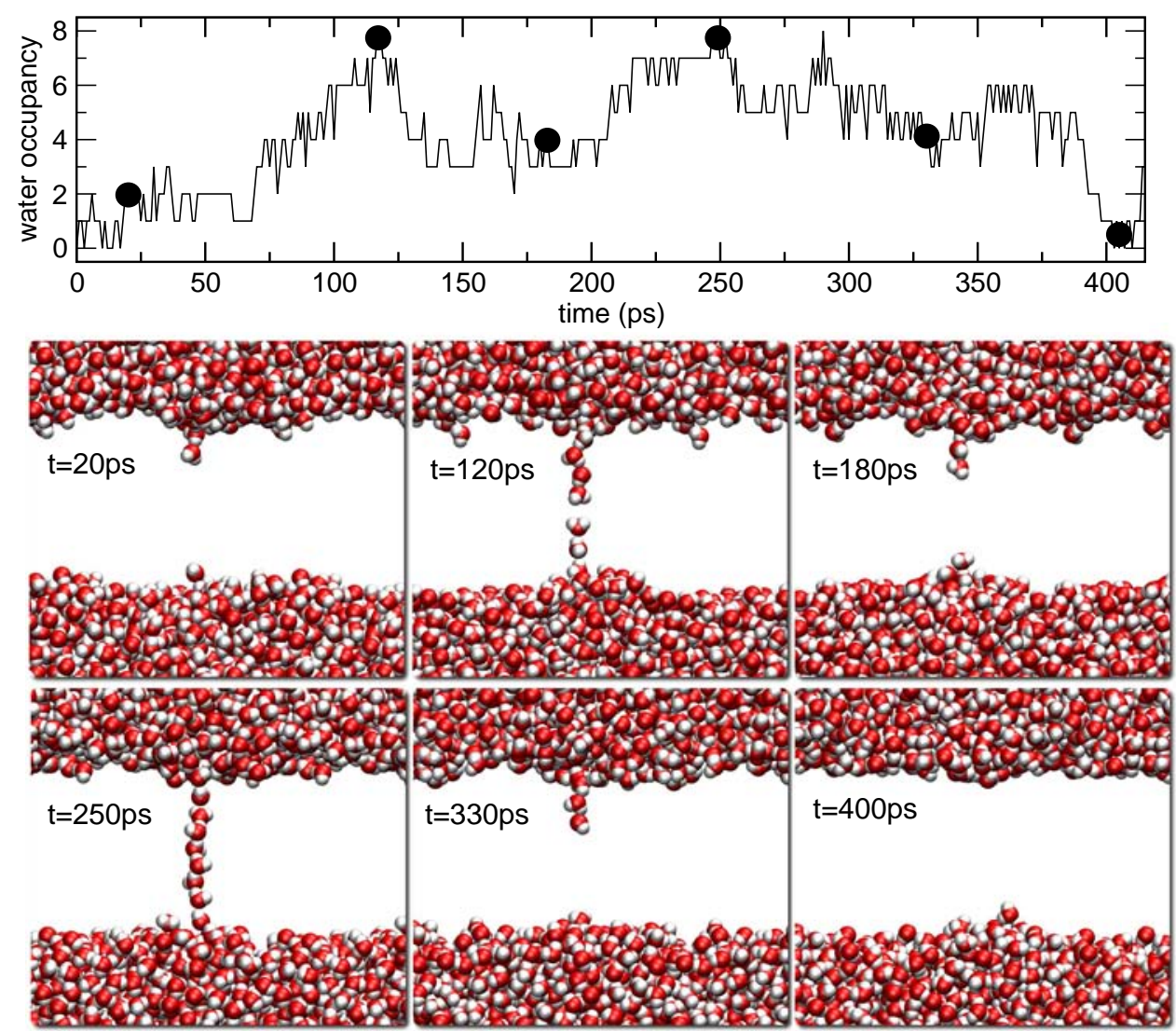

Figure 4.9.: Illustration of typical water filling events in low polarity $(2.01 \mathrm{D})$ peptidic pores. The upper panel shows the time evolution of water molecules occupancy in the p-27 pore. The black dots denote the time were snapshots were extracted from the simulation (lower panel). The peptide and the membrane are not shown for clarity. The sequence was extracted from an equilibrated trajectory.

channels are ion channels, and ions do not cross single-file pores without solvation $[155,156]$, the integrity of the water column increases the probability of ion translocation.

Permeability coefficients as function of backbone polarity The pore polarity affects the water permeability as a result of two distinct contributions: the probability for a water molecule to be inside the pore and the diffusivity of the water molecule once it is in the pore. The first factor is directly related to the averaged occupancy, which depends on the polarity of the pore in the functional form detailed in the previous section. In a Kramer's-like activated process, the 


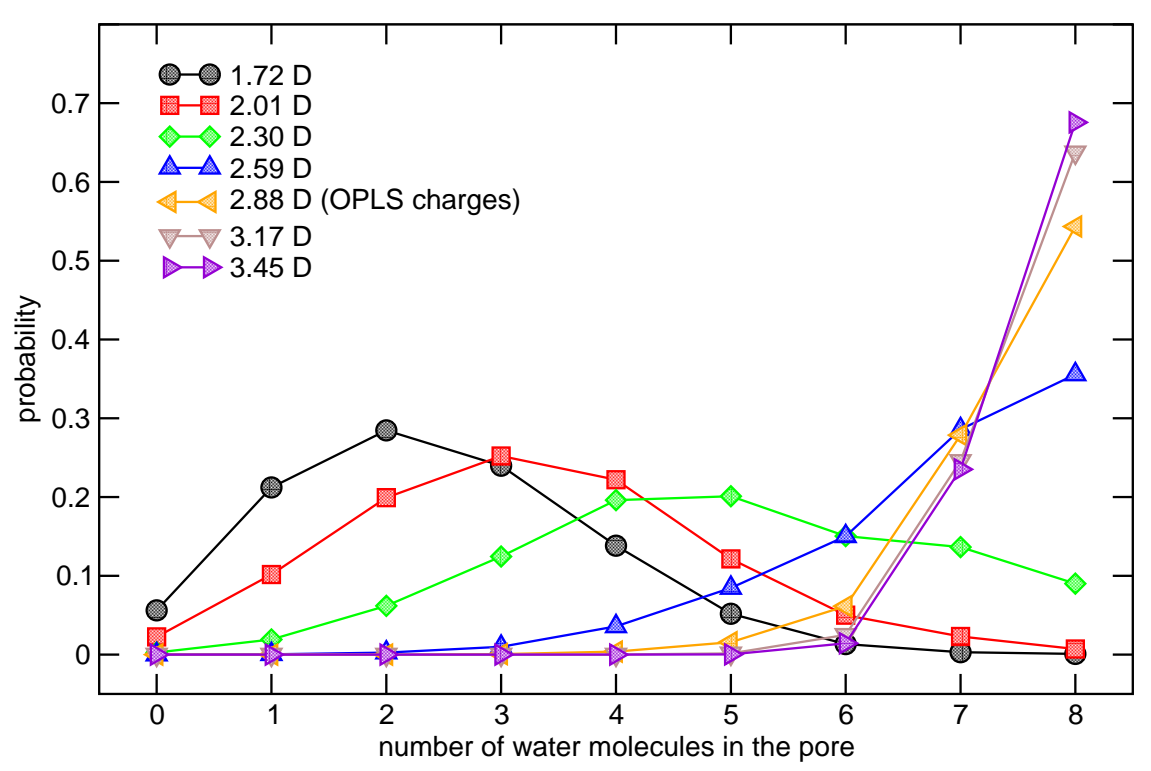

Figure 4.10.: Probability distribution of number of water molecules inside the p-27 peptidic pore for different dipole moments of peptide backbone carbonyl groups. The probabilities have been normalized to the length of the simulation.

diffusivity can be related to the curvature of the underlying energy landscape.

Here, we characterized the flux of water molecules in the form of permeability coefficients. The osmotic permeability coefficient, $\mathrm{p}_{f}$, was computed as the frequency of water-water jumps along the pore, averaged over three equidistant regions inside the pore. The diffusive permeability coefficient, $\mathrm{p}_{d}$, was computed as the number of complete water translocation from one compartment to the other per unit of time, as derived in 2.2.2.

Osmotic and diffusive permeability coefficients are displayed in Fig. 4.11 as function of the dipole of the peptidic carbonyl groups. The most striking consequence of changing the polarity of the peptidic backbone is the large variation of permeability coefficients, both osmotic and diffusive. Changing the dipole moment of the peptide backbone carbonyl groups from $1.72 \mathrm{D}$ to $2.59 \mathrm{D}$ increases the osmotic permeability coefficient $\sim 5$ fold. A further increase of pore polarity to $3.45 \mathrm{D}$ decreases the osmotic permeability coefficients by roughly the same amount. These results agree very well with previous analytic models [157].

As observed for the OPLS charges, the osmotic permeability coefficient is effectively independent of the length of the water column. Even though the 


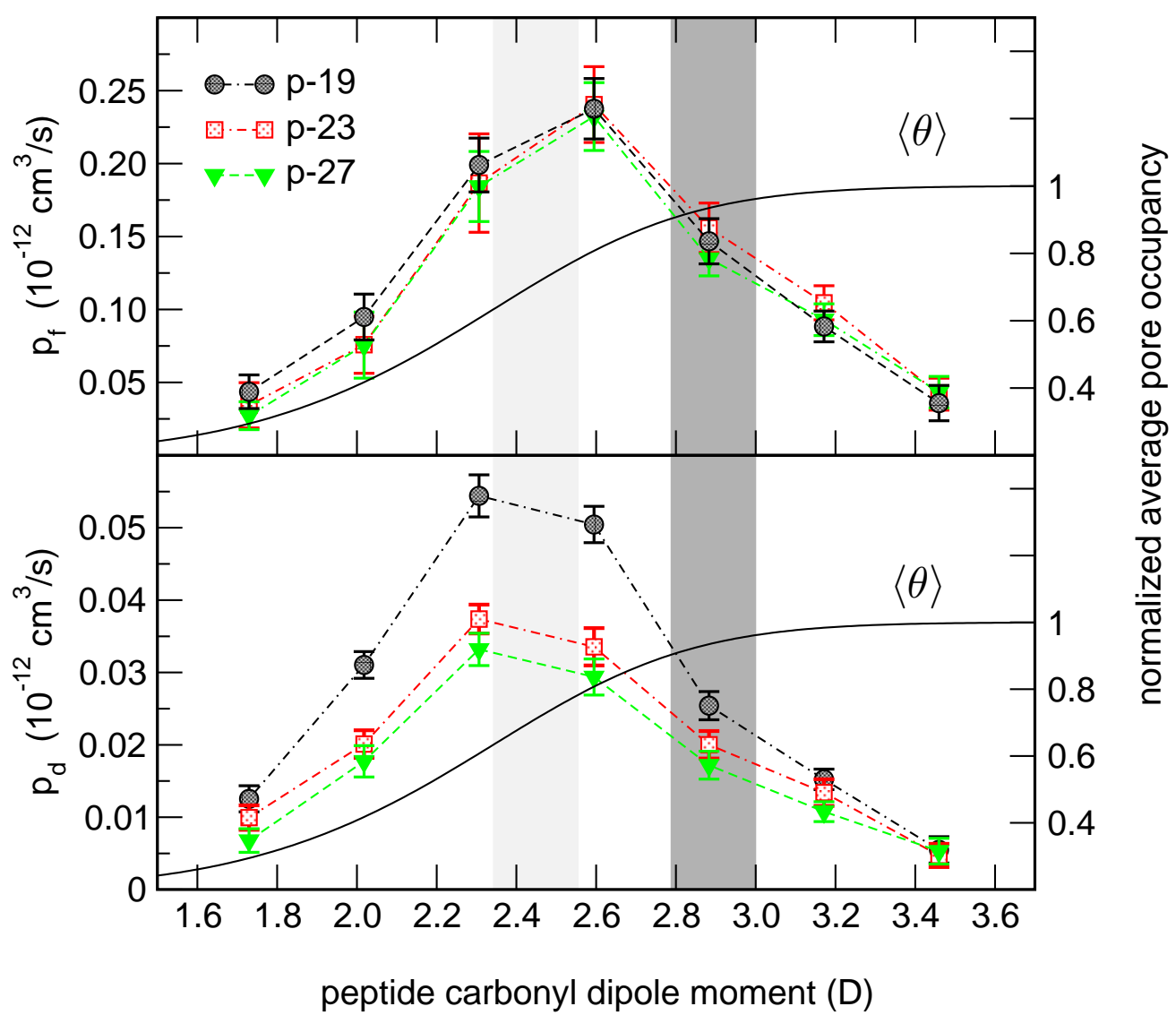

Figure 4.11.: Osmotic, $p_{f}$, and diffusive, $p_{d}$, permeability coefficients as function of the dipole moment of the peptide backbone carbonyl group for the three channels studied, averaged over two independent simulations for each polarity. The fitted averaged occupancy $\langle\theta\rangle$ at each polarity is displayed as a continuous black line. The light gray area indicates the polarity at which the permeability is the highest, correlated with intermediate backbone polarity and medium water occupancies. The dark gray indicates the dipole corresponding to the OPLS charges.

behavior of water inside the pore changes with polarity, as seen by the results presented in the previous section, the mobility of the water molecules does not strongly depend on the length of the water column within the studied range of channel lengths. This finding is consistent with the computed normalized water occupancy as function of the backbone polarity, which was also independent of the channel length.

The diffusive permeability coefficient, related to the number of water molecules that completely cross the pores, decreases as the channel length grows. The most significant difference occurs at intermediate polarities, analogous to the $p_{f}$, due 
to the greatest variation in mobility of the water molecules.

To summarize, the emerging picture is that: the probability to find a particle in the pore at low polarities is small due to the great difference in water chemical potential. As the pore becomes more hydrophilic the channel gets more populated (see the thin black curve in Fig. 4.11), therefore more water can permeate. At an intermediate polarity (in this case around $2.5 \mathrm{D}$ ) the pore becomes almost full, and the pore-water affinity allows fast hops of the water column. At further increase of the water-pore affinity leads to stronger water-channel interactions, and hence, sticky channels, effectively preventing rapid hops of the water column. The pore occupancy that leads to a maximal flux is not $50 \%$, as can be seen from Fig. 4.11. Rather, the optimal pore occupancy for efficient water transport is located in the vicinity of the change in slope of the curve relating the occupancy with the polarity, at $\sim 75 \%$ pore occupancy.

The ratio of $\mathbf{p}_{\mathbf{f}}$ to $\mathbf{p}_{\mathbf{d}}$ and the proportionality to $\langle\mathbf{n}\rangle$ As discussed earlier (pg. 22), the ratio of osmotic and diffusive permeation coefficient in single-file pores is proportional to the water occupancy $n$, namely $p_{f} / p_{d}=n+1$. In the mechanistic view of water molecules jumping from binding site to binding site, the $n+1$ factor results from the fact that it takes $n+1$ jumps across $n$ water positions to fully cross the channel. The water jumps by one water-water distance are proportional to the $p_{f}$, and the number of complete water translocations is proportional to the $p_{d}$.

Figure 4.12 shows the ratio of osmotic permeabilities (minus one) as a function of the averaged pore occupancy. The straight black line represents the $\langle n\rangle+1$ result. As can be seen, the prediction of the occupancy is fulfilled for all channels and polarities. The highest (but insignificant) deviations from the $\langle n\rangle+1$ ratio occur at high and very low polarities, which can be attributed to the low number of water passage under these conditions. This remarkable finding shows that the relationship 2.25 also holds for partially filled channels, when $n$ is replaced by $\langle n\rangle$. The ratio of osmotic and diffusive permeabilities should therefore be expressed as $p_{f} / p_{d}=\langle n\rangle+1$, implying that it captures the averaged occupancy. This result allows the experimental detection of liquid-vapor oscillations in single-file channels. Occupancies can be computed by measuring the averaged number of water molecules using equation 2.25, and the degree of occupancy 


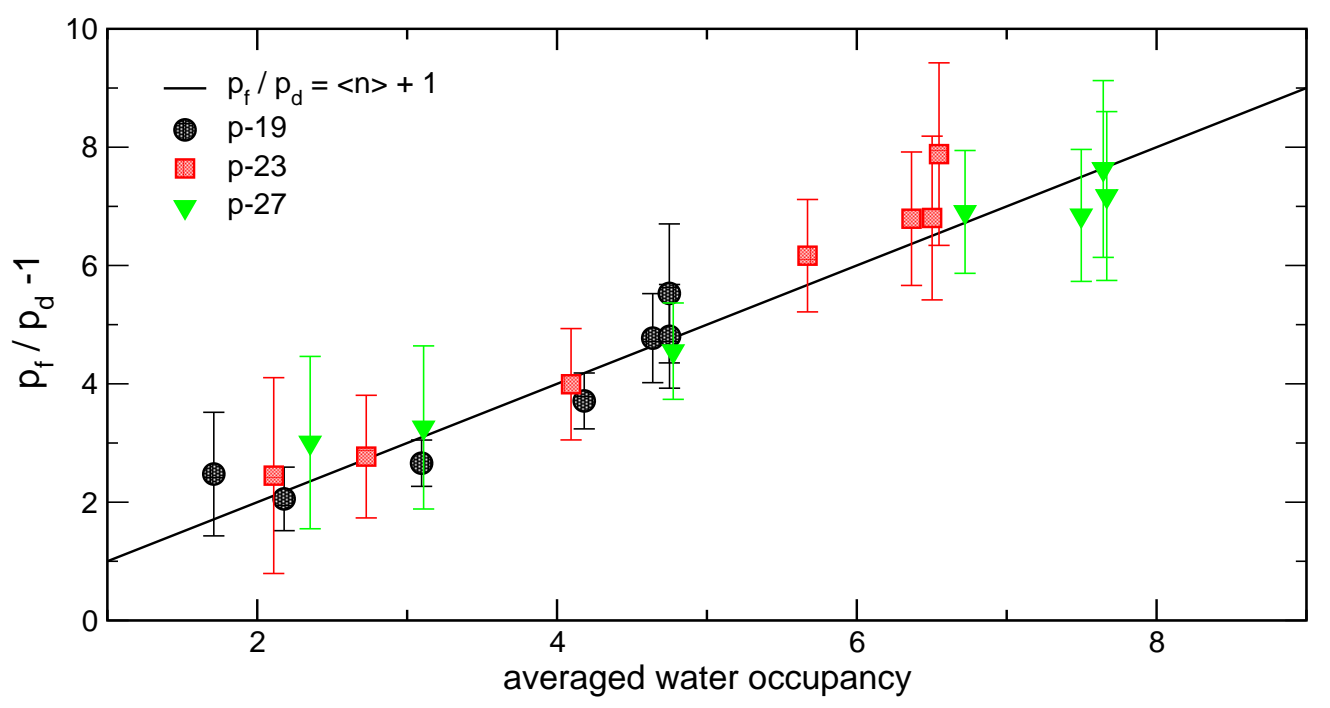

Figure 4.12.: The ratio of osmotic and diffusive permeation coefficients, $p_{f} / p_{d}$, is linearly proportional to the averaged water occupancy in the single-file peptidic pores for any peptide backbone polarity.

on the maximum number of water molecules that geometrically fit in the pore. If the channel shows strong occupancy fluctuations, the observed permeability ratio would be significantly smaller that the maximum occupancy allowed by the channel length.

\subsubsection{Summary and Conclusions}

We addressed the effect of pore polarity on the pore length dependence of water permeation in single-file channels. To this end, we extended the study of the permeability coefficients to different backbone polarities for a subset of the previously introduced polyalanine channels. To modify the polarity of the pore we changed the dipole moment of the carbonyl groups forming the peptide backbone, thus keeping the pore relatively hydrophilic even at low values of the carbonyl dipole.

The time-averaged pore occupancy as a function of pore polarity was found to follow a sigmoidal curve. We modeled the occupancies by computing the equilibrium constant between vacant and occupied positions, governed by a free energy quadratically dependent on the dipole moment of the carbonyl groups. At low polarities we observed strongly fluctuating water occupancies in the pore, 
leading to broad distributions of number of water molecules inside the channel (Fig. 4.10). Moving towards high polarities rises the water occupancy and narrows the distribution of the number of water molecules inside the pore.

The effect of the backbone polarity on the permeation coefficients was found to be remarkably strong. The OPLS charges, which closely mimic the polarity of naturally occurring aminoacids, were found to be almost optimal for water permeation. The bell-shaped dependence of the osmotic and diffusive permeability coefficients can be explained by simple intuitive arguments: low polarities imply low occupancies, but fast fluxes once the water molecules are in the pore. Increasing the polarity rises the average occupancy allowing more molecules to permeate within a given time frame. At further increase of the water poreaffinity prevents fast hops of water molecules, and effectively reduces the water flux. Consequently, the maximum flux was found to be at intermediate pore affinities. This simple structure-activity relationship reminds of the underlying energetics in catalysis: the host needs to stabilize the guest molecules to be able to perform its activity, but the binding energy should not be too high to block the release of the products.

Within the length spanned by these channels, comparable to the range covered by the mini, midi and gramicidin channels, no major effects on the channel mobility were observed as function of the length. The greater difference in diffusive permeability coefficients takes place at intermediate pore polarity, where the mobility of the water molecules is the highest. These results show that in different permeation regimes the mobility of water molecules in single-file peptidic pores does not depend on the channel length.

Based on the results of this study, naturally occurring channels with a pore spanned by carbonyl groups, such as gramicidin derivatives or potassium channels homologues, are found to display a fine-tuned hydrophilicity that allows fast permeation of water molecules. Lower polarities of the peptidic backbone would result in intermittent open/closed channels, impeding the transport of water and ionic species. Large water-channel attraction would drastically reduce water mobility, also decreasing the pore activity.

The basic knowledge on the polarity effect on water permeation in single-file pores can be readily used to devise custom water channels. Since the channel length is not a major determinant, the channel can be made long enough to 
match the membrane slab. Peptide derivatives with altered sequences to include polar/apolar fragments could be designed to match the desired water affinity, thus modulating the water permeability. 


\section{Influence of the pore radius and polarity on the water permeability}

\section{Summary}

In this chapter, we continue the systematic analysis of the channel characteristics that affect the water permeability through pores of molecular dimensions. Naturally occurring pores show a variety of polarities and sizes that are presumably directly related to their biological role. The understanding of this relationship yields valuable insights not only in the function of biological channels but also for the design of channels with specific properties. Therefore, we explore the effect of the polarity and the radius on the water permeability through pores of such dimensions.

Since many biological channels are selective towards permeants similar or smaller in size than water molecules, their pores operate in the single-file regime. Pores that conduct water in a single-file fashion span a range around the radius of one water molecule. Within this range, and depending on the polarity of the channel, the population of water molecules inside the pore displays different time evolutions $[31,32]$. Our focus here is put on the characterization of the transition between different permeation regimes in terms of the structure of water in the pores, the average pore occupancy and the dynamics of the permeating water molecules. We studied pore sizes up to $0.55 \mathrm{~nm}$, to follow a gradual approach to the regime of hydrodynamic laws observed in macroscopic pores. Since there is no formal theory that describes the radial dependence of the permeability for any pore size and polarity, the model systems and the methodology used in this work represent a valuable step in this direction. 


\subsection{Introduction and previous studies}

Some of the necessary concepts required to understand the discussion in this chapter were introduced in section 2.2 of chapter 2. Early studies on the relation between the osmotic and diffusive permeation through membranes led to the discovery and characterization of water pores in membranes [5]. To establish a relation between the measured permeabilities and size of the pore, macroscopic hydrodynamic equations were modified [158, 159] to account for the diffusive nature of the permeability coefficients. Such modifications were empirical and ad hoc, rather than based on solid physical theories, and the radii obtained from their applications were regarded as an effective radius or equivalent radius [5] that fitted into that model. The single-file regime was, however, believed to be fully understood and properly characterized using thermodynamic arguments. However, the theory does not distinguish explicitly between different pore radii that allow water molecules to permeate in single-file. The theoretical description of the single-file transport revealed characteristic diffusive properties [160], like a mean squared displacement proportional to the square root of the time [161-163], but no direct relationship with the size of the pore was put forward.

Molecular dynamics and Monte Carlo simulations offer a direct view at the motions of particles in such microscopic pores. Levitt pioneered the computational study of hard-spheres in a cylindrical pore [57]. Recently, more realistic computational strategies modeled the structural and dynamical properties of water in nanoscopic model pores [31-33, 61, 150, 155, 164, 165], with special attention to the newly discovered burst-like permeations in hydrophobic channels. While Beckstein et al. [32, 155] devoted much of the attention to the geometry and polarity of nanopores towards the understanding of the hydrophobic contribution to ion and water gating, the water occupancy rather than the flux was systematically investigated. Similarly, previous work by Allen et al. [164] focused on the structural and diffusive properties of water molecules in nanopores, and a systematic study of their osmotic permeability dependence was not performed.

The simulations presented here corroborate previous findings, such as liquidvapor oscillations for narrow hydrophobic pores and low diffusivity in the singlefile regime, while offering detailed insight into the dependence of permeability coefficients on the pore radius. 


\subsection{Theory and Methods}

Design of model channels The design of stable and functional membrane integral peptides that form pores of desired radius is a challenge on its own. To concentrate on the geometrical and electrostatic properties of the pore, we therefore use simplified channels with helical topology of well defined radius and length. The shape of the channel is based on the $\beta$-helix conformation of gramicidin A in the helical dimer. Modeling a peptidic sequence arranged in a $\beta$-helix has the constraint of inter turn hydrogen bond pattern, which fixes the available radii. Since the goal here is to study pores with varying radius, we move away from peptide sequences. Instead, we built chains of pseudo atoms to form the pore walls. The use of helices to model the pore with a finite number of particles (atoms) allows any value of the radius for a given height, rise per turn and bond lengths.

To mimic the hydrophilic environment of peptidic pores, basically due to carbonyl and amino groups, we used oxygen-like and carbon-like pseudo atoms. These pseudo atoms, even in number to guarantee the neutrality of the chain, are arranged alternatively and connected with bonds. A membrane slab of octane molecules was used to have full control over the match between the pore and the membrane, as described before (pg. 47). To guarantee a good match with the octane membrane, the helices were surrounded by a second shell of carbon atoms of the same particle type as the octane molecules that form the membrane (depicted as green balls in Fig. 5.1). The stability of the helix was ensured by using position restraints $\left(1000 \mathrm{~kJ} / \mathrm{mol} / \mathrm{nm}^{2}\right.$ harmonic force constant) to the initial reference structure of the pore.

Eighteen helices of the same length $(1.8 \mathrm{~nm})$ and increasing radius were prepared. The rise-per-turn height was fixed to $0.45 \mathrm{~nm}$, which is similar to the backbone-backbone rise-per-turn distance in gramicidin A in a helical dimer conformation. The analytical radius of the pore is defined as the distance between a particle of the pore and the center of mass of the pore, and ranges from 0.36 $\mathrm{nm}$ to $0.78 \mathrm{~nm}$. The effective radius, $R_{\text {eff }}$, and rise-per-turn distance are much smaller due to the volume excluded by the atoms, and due to attractive/repulsive interactions also depends on the charge of the pore. Furthermore, the helical topology imposes a sinusoidal cross-section along the pore. Due to these effects, the effective radially averaged radii used in this study range from 0.13 to 0.54 
nm.

Each pore of different radius was simulated with 7 different pore polarities. The partial charges of the carbon pseudo atoms were increased from +0.3 to $+0.6 \mathrm{e}$ in steps of $0.05 \mathrm{e}$, with a corresponding decrease from -0.3 to $-0.6 \mathrm{e}$ for the oxygen pseudo atoms. Series of channels with the same partial charges are named ch-030, ch-035, ch-040, etc...

The same protocol applied to construct the initial simulation boxes for the polyalanine channels was followed, see Section 4.1.2. As before, the interactions between all atoms of the pore and the membrane were described by the OPLSAA force-field, and the TIP4P water model was used for the solvent. All simulations were performed using the GROMACS 3.3.1 simulation software [134, 135], with PME for long-range electrostatic treatment and a cut-off radius of $1 \mathrm{~nm}$ for short-range repulsive and attractive dispersion interactions, modeled via a Lennard-Jones potential. Short simulations ( 300 ps) with anisotropic pressure coupling were run to equilibrate the membrane-pore interface. The simulations for production were performed at constant volume to preserve the integrity of the membrane. Each combination of pore radius and pore charge was simulated for 100 ns. Discarding the first nanosecond as equilibration, the complete trajectories were used for all the analysis performed. Pore dimensions were estimated from the individual molecular dynamics trajectories using the program HOLE [137]. The total simulation time amounted to $12.6 \mu \mathrm{s}$.

Radial distribution function of water molecules in the pore This function describes the local environment of any reference particles, in this case the oxygen atoms of the water molecules, in terms of the probability to find a neighboring particle located at a distance $r$. The radial distribution function $g(r)$ is usually defined such that it approximates to 1 at large distances,

$$
g(r)=\frac{\langle\rho(r)\rangle}{\langle\rho\rangle}
$$

where $\langle\rho(r)\rangle$ is the density of particles at a distance $r$ and $\langle\rho\rangle$ is the system density.

To compute the RDF of a water molecule inside the pore, we must first recognize that not all positions are equivalent. To encompass the largest number of 
particles in the pore, we constrained our analysis to the center of the channel. As reference particles we used the water molecules located in a section of $0.15 \mathrm{~nm}$ in height, positioned at the center of the pore. From those reference particles, all the distances to the rest of the water molecules in the pore are computed. Collecting the number of neighbors at a given radius interval $\delta r$, normalized to the appropriate corresponding volume $\delta V$, yields the $\langle\rho(r)\rangle$. To properly account for the restricting geometry of the pore, the normalization volumes were computed as the intersecting volume between a sphere centered at the reference particle and the cylinder defined by the channel. Finally, the densities at distance $r$ are normalized to the density of unique water molecule pairs inside the channel. Due to the short size of channels and their anisotropy as a system, the radial distribution function does not decay towards the averaged channel density.
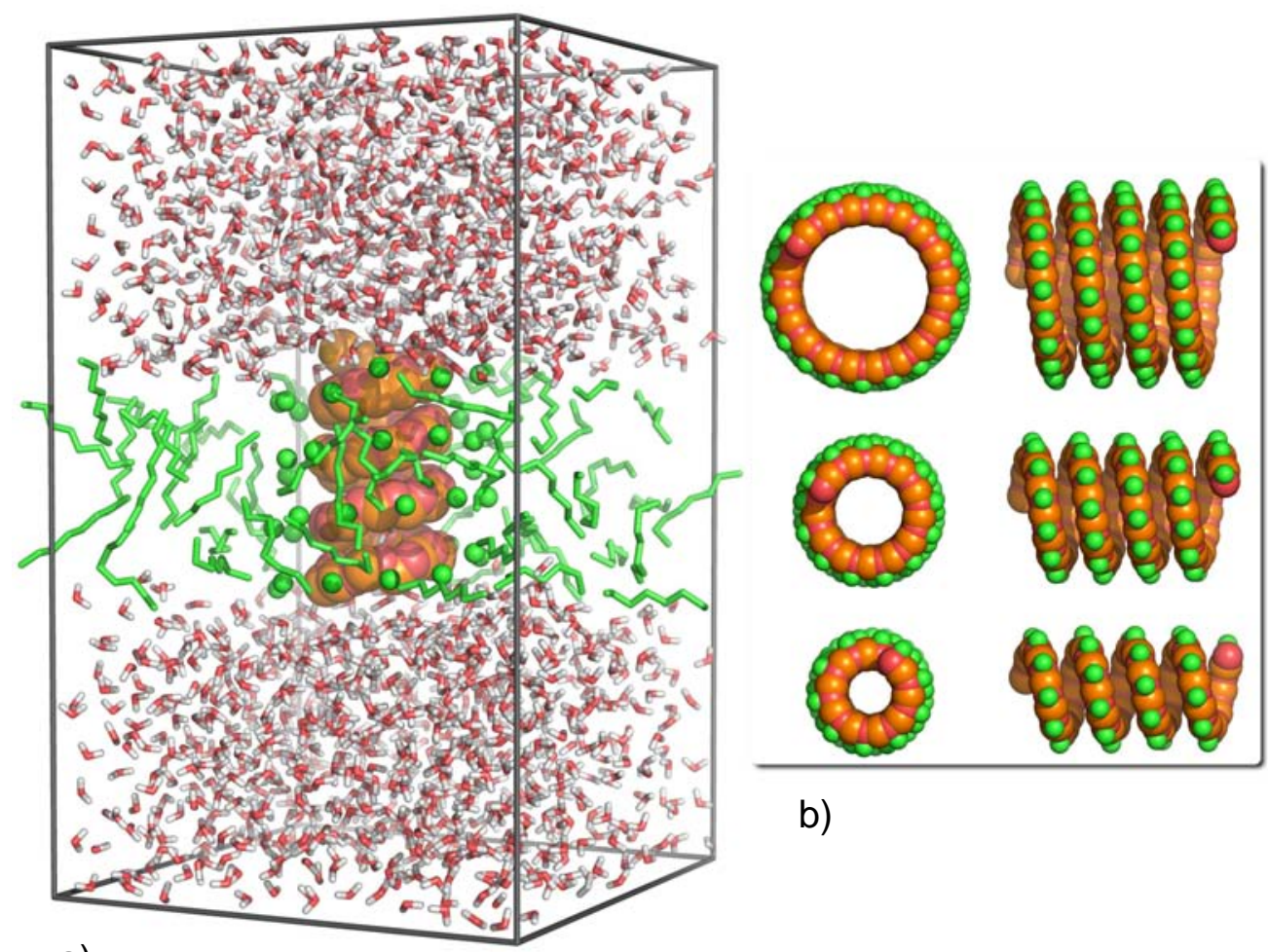

b)

a)

Figure 5.1.: a) Sketch of a typical simulation box. The octane molecules are drawn as green sticks and the octane-like atoms connected to the pore as green balls. The pore is drawn as red and orange balls. b) Top and side view of three designed channels of different radius (from top to bottom: $0.16 \mathrm{~nm}, 0.30, \mathrm{~nm}$ and $0.55 \mathrm{~nm}$ ) and length $1.8 \mathrm{~nm}$. 


\subsection{Pore water occupancies and density as function of radius and pore polarity}

The analysis of the pore occupancies as a function of the pore radius and the pore charge can be carried out in analogy with the study performed in subsection 4.2.1. In this case, however, the maximum occupancy possible for a given channel length changes with increasing radius. To circumvent this, Beckstein [32] chose to describe the state of hydrophobic pores as an equilibrium between open or closed states, depending on the water density inside the pore.

Since our pores are mainly hydrophilic, the so-called liquid-vapor oscillations are only visible for narrow pores with an effective radius up to $0.16 \mathrm{~nm}$. A two state description, open or closed, would therefore not be discriminative enough: most of them can be considered fully open because at $0.15 \mathrm{~nm}$ all pores show densities above half the bulk water density (Fig. 5.2 panel c). Therefore, our analysis of pore occupancies is done based on average occupancies $\langle n\rangle$ and water densities $\rho$, rather than normalized occupancies $\langle\theta\rangle$, as defined in Sec. 4.2.1, or the openness of the pore [32].

Figure 5.2 displays the water occupancy (panel a and b) and the water density (panel c) in pores of different radius and polarity. As a general feature for all pore radii, higher channel polarity results in a higher occupancies of water molecules. At small pore radii, below $0.15 \mathrm{~nm}$, the channel is only occupied at high pore polarities (see inset Fig. 5.2). At this range of radii the increase of pore occupancy is exponential with increasing pore radius, and the averaged occupancy reflects time fractions of open/closed states, similar to what was reported in Sec. 4.2.1 for apolar polyalanine channels and previously in [32]. The increase ceases after the highest packing configuration is reached, indicated by the first maximum of the density as function of the pore radius (Fig. 5.2 panel c). For these single-file pores, the maximal of the pore water density occurs when the pores reach a fully occupied single-file (no gaps), corresponding to $\sim 3.63$ water molecules per $\mathrm{nm}$. The radius at which the density reaches a local maximum depends on the polarity of the pore: more charged pores, with a higher water affinity, reach the fully occupied single-file configuration at a lower radius than less polar pores.

Beyond the optimal single-file packing, the available pore section increases faster than the occupancy. As the channel radius grows, the water molecules start 


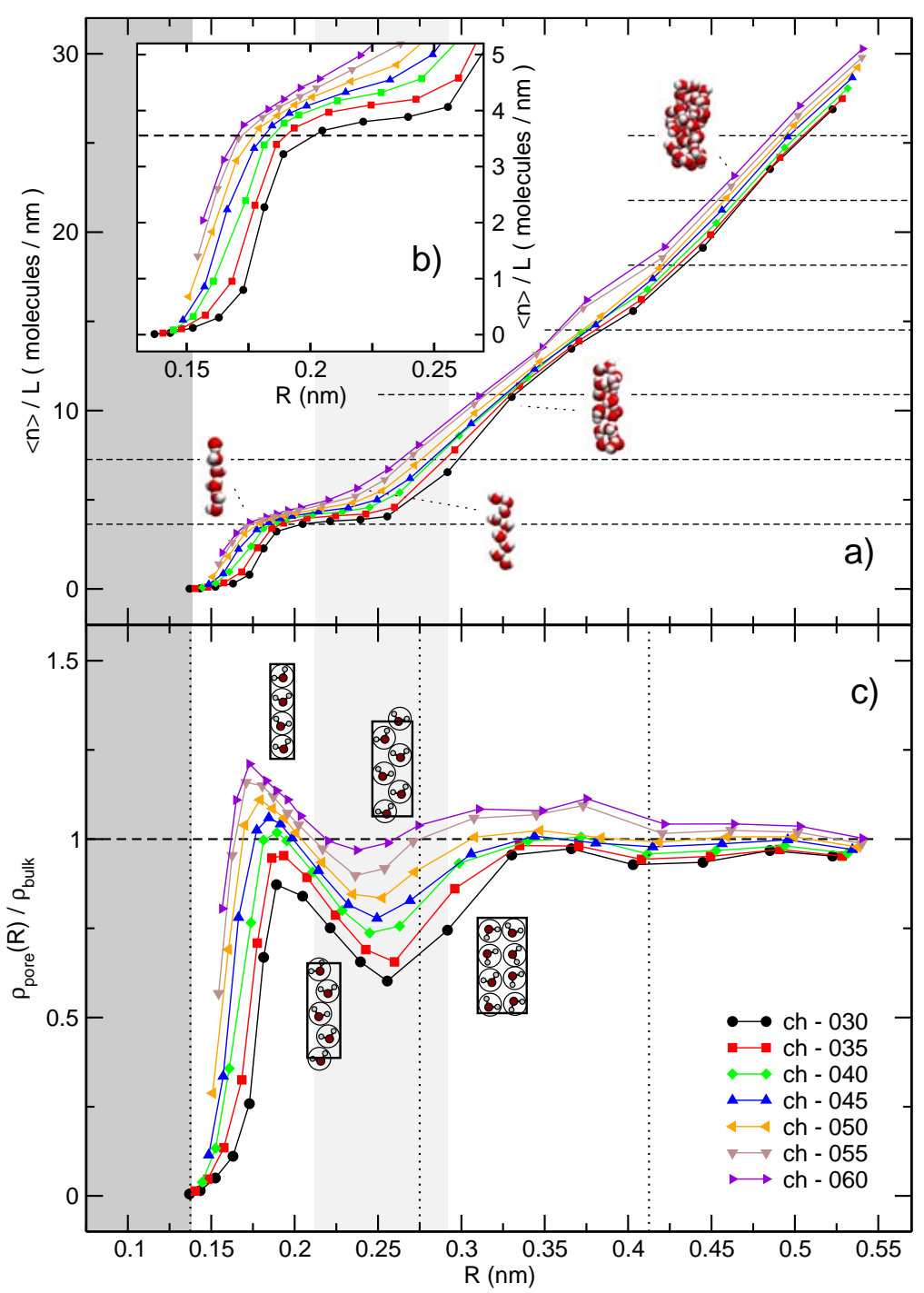

Figure 5.2.: a) and b) Pore water occupancy as a function of the pore radius for different pore polarities. For direct comparison, the water occupancies at each radius were divided by the pore length. The horizontal dashed lines indicate multiples of 3.63 water molecules per $\mathrm{nm}$, the pore occupancy for a perfectly packed single-file. The inset is a detailed view of the occupancies at small pore radius. c) Pore water density at different pore radius and pore polarities. The dark gray area indicates radius smaller than the typical water molecule radius $(\sim 0.137 \mathrm{~nm})$, and the light gray area displays the minimum in the water density profile that correlates with the transition from/to the single-file regime. The vertical dashed lines mark the radii corresponding to an integer number of water molecules. 
positioning in a helical pattern, that projects along an axial pore plane as a zigzag pattern, see for example Fig. 5.4 panel b. Since the new configuration allows only a small additional water occupancy compared to the volume gained by the increase of the radius, the water density drops. This behavior is due to the discrete nature of water molecules, and it is sketched in Fig. 5.2 panel c. In this range of radii, we can assume that the occupancy increases linearly with the radius (Fig. 5.2 panel b), thus the density drops as $\sim\left\langle n_{\text {max. single file }}\right\rangle / R_{\text {eff }}^{2}+\mathcal{O}\left(1 / R_{\text {eff }}\right)$. At a radius slightly smaller than the water molecule diameter, an inflection point in the occupancy and the density curve occurs: it corresponds to the transition from/to single-file channel. After this point the helical arrangement of water molecules can no longer be characterized by a no-pass condition. Due to the intrinsic motions of the pore, partially non-single file structures can form before the average pore radius exceeds the diameter of a water molecule. As seen from both the channel densities and occupancies, an increase in channel hydrophilicity results in smoother transitions between the two regimes.

The double-file configuration is not a local maximum of the density as afunction of the pore radius. Here as well, the density change with respect to the pore radius is more pronounced for the low-polarity pores, e. g., in channel ch-030 there is a transition from double-file to triple-file within $\sim 0.02 \mathrm{~nm}$, whereas it requires $\sim 0.04 \mathrm{~nm}$ for ch-060. After reaching a quadruple-file (at a radius between $0.36 \mathrm{~nm}$ and $0.4 \mathrm{~nm}$ depending on the polarity) the oscillations in channel density as function of the pore radius damp and converge towards the density of bulk water. After $\sim 0.4 \mathrm{~nm}$ the water occupancy in channels of different polarity can be approximated by $\rho_{0} V_{\mathrm{ch}}$, where $\rho_{0}$ is the density of bulk water and $V_{\mathrm{ch}}$ is the available channel volume.

Structural arrangement of water molecules in the pore To further illustrate the effect of pore polarity on the occupancy, Fig. 5.3 displays the normalized radial probability density for a water molecule in pores of the same radius $(\sim 0.18$ $\mathrm{nm}$ ) and different polarity. The radially averaged densities, centered at the channel main axis, were also averaged over the length of the pore. The most probable position of a water molecule is located closer to the channel wall the more polar the pore is, a feature also observed in [164]. The shift of the most likely position towards the walls of the pore allows the system to fill the channel more 
efficiently: the overall water density in the channel is higher for the pores with higher charges. For the same reason the transition from and to the single-file regime occurs at a higher pore radius for the low polarity pores: water molecules tend to cluster via hydrogen bonds in spite of the increase of available area. Since the pore helical shape was constrained to a given length, regardless of the pore radius, they are not fully axially symmetric with respect to the center of mass. In this case the radially averaged density is not a robust criteria to detect the transition from and to single-file regime, but it serves us to observe the effect of pore polarity on the preferred radial distribution for a given radius.

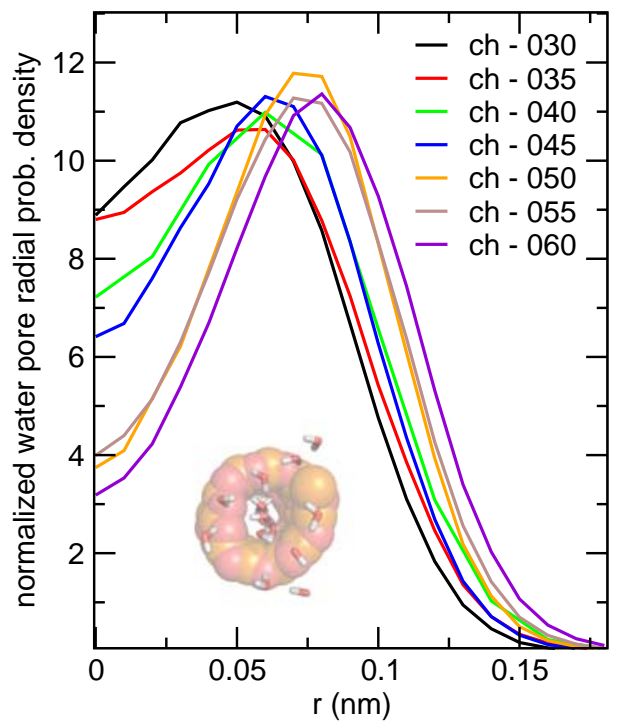

Figure 5.3: Normalized radial probability density for channels of given pore radius $(\sim 0.18 \mathrm{~nm})$ at different pore polarities. As the polarity of the pore increases the maximum of the probability density is shifted towards the wall of the pore. The drawing illustrates a top view of a singlefile pore.

So far, we have described the transition from and to different transport regimes focusing on the channel as a subsystem. To expand the characterization of the transitions, we studied the structural configurations of water in the channels by means of the radial distribution function (RDF), or pair distribution function (equation 5.1).

Figure 5.4 displays radial distribution functions for a water molecule in the pore center for three channel polarities at different radius. The RDF of bulk water, the black dashed curve, is included for comparison. For all RDFs the first peak is located at the same $r$ value $(0.273 \mathrm{~nm})$, which corresponds to the first shell of water molecules. The red curves show the RDF for the single-file regime of maximum water density. The peak pattern is characteristic of the single-file regime: each maximum is located at an integer number times the water-water distance. As the channel becomes more polar the height of the first 
peak increases. This finding is consistent with the higher water densities in polar channels presented in Fig. 5.2 panel c.
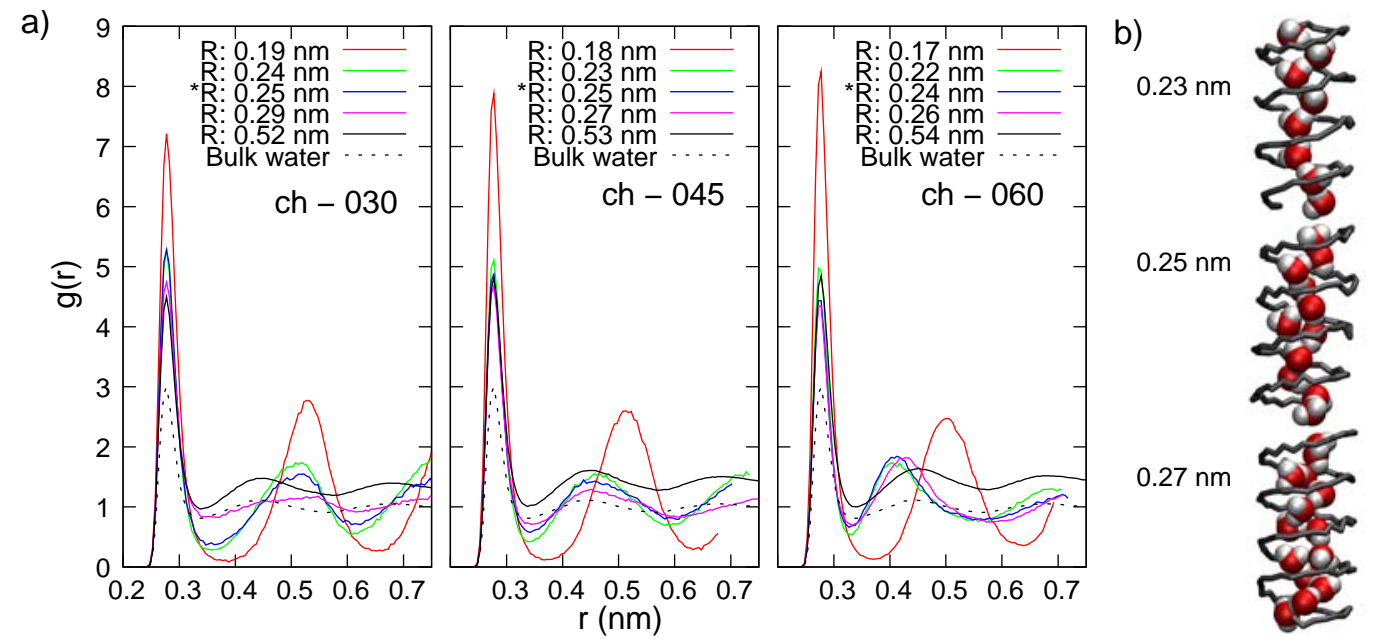

Figure 5.4.: a) Radial distribution function $g(r)$ (RDF) for water molecules inside the pore as function of the effective pore radius at three different pore polarities (ch-030, ch-045 and ch060). The red curves show the RDF at the radius corresponding to the highest water density. The green, blue and magenta curves illustrate the transition from single-file (green) to non single-file (black). The cyan curve indicates the RDF at the largest pore radius studied, which has the characteristic extrema pattern as the bulk water (black dashed curve). b) Illustration of the transition from single-file regime (upper channel) towards double-file (lower channel) for the ch-045 pore polarity.

As the radius of the pore increases, the second peak lowers its relative density and moves towards shorter distances, indicating that a zigzag pattern emerges. The green curve is the RDF for the radius previous to the transition, the blue curve is the RDF corresponding to the radius at which the density displays a local minimum, i.e., where the transition to a double-file occurs. The magenta curve represents the $\mathrm{RDF}$ at a radius where the double-file is the predominant configuration. Finally, the pattern of extrema at large radii (black curve) is the same as in bulk water (dashed black curve), although the absolute value of $g(r)$ differs due to the confined geometry of the pore.

The change in position of the second peak is a robust indicator of the transition from and to a single-file in pores with low polarity. Since the water molecules tend to lie at the center of the pore in channels with low polarity, as seen in Fig. 5.3, the second peak is located very close to twice the water-water distance $(0.55 \mathrm{~nm})$. As the channel becomes more hydrophilic, the water molecules move towards the walls of the pore, thereby shifting the second maximum to shorter distances. For 
pores of higher polarity than ch-045 the transition becomes smoother in terms of the RDF.

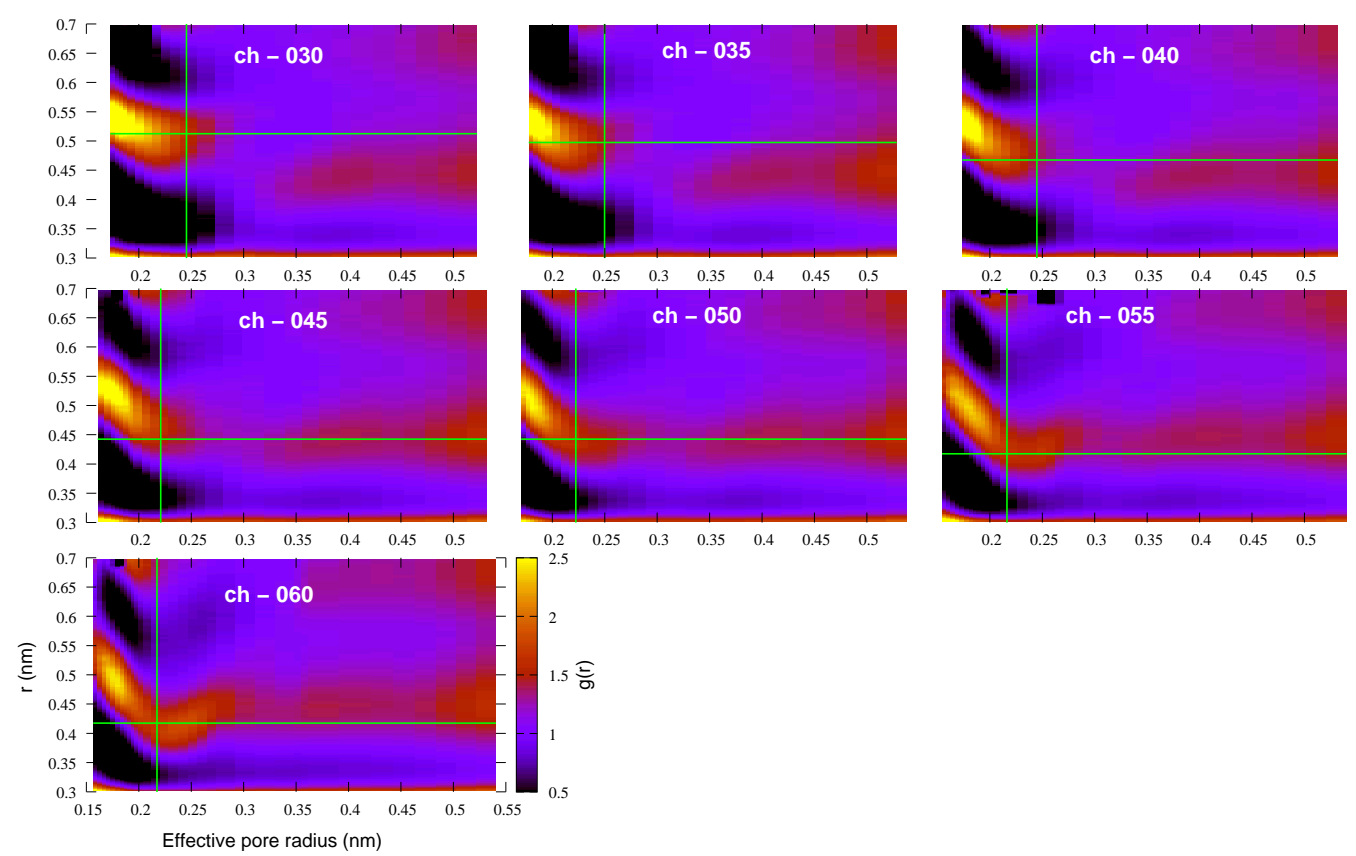

Figure 5.5.: Radial distribution function $g(r)$ (RDF) for water molecules inside the pore as function of the effective pore radius at different pore polarities (ch-030 to ch-060). The plots show the area beyond the first peak in $g(r)$, located at $0.275 \mathrm{~nm}$. The green lines indicate the position at which the single-file vanishes, see the text for further details. At large effective pore radius the $R D F$ approaches the features of bulk water, with peaks at $0.44 \mathrm{~nm}$ and $0.77 \mathrm{~nm}$.

The drift of the second maximum in the radial distribution function is illustrated in Fig. 5.5, which shows the RDF as a function of the channel radius for all channel polarities. The RDF is plotted after the first maximum to clearly resolve the oscillations at small $g(r)$ values. As the channel polarity increases, the second maximum moves closer to the reference water molecules when the pores become wider. The position of the transition involving the single-file regime is indicated by green lines: the horizontal line marks the distance of the second peak with respect to the reference water, and the vertical line indicates the radius at which the transition is happening. The transit in between the two regimes occurs at a pore radius for which the second maximum in the RDF is located closest to the reference water molecules, and before the drop in $g(r)$ of the second maxima 
takes place (at $\sim 0.30 \mathrm{~nm}$ for ch-030 to ch-050, and at $\sim 0.32 \mathrm{~nm}$ for ch-055 and ch-060). As already seen in Fig. 5.4, at a large radius all profiles converge to the same pattern, with extrema at the same position as in bulk water.

To summarize this section, the single-file regime in hydrophilic pores covers a range of $0.137 \mathrm{~nm}$ in which the pore occupancy is monotonically increasing with the radius, whereas the pore density presents a maximum. The first minimum in the density as a function of the radius indicates the transition from/to a single-file regime. Beyond the single-file a small increase in the pore radius allows a fast transition from a double to a quadruple file. The water density in the pore converges to the bulk water density at radii larger than $\sim 0.4 \mathrm{~nm}$. The polarity of the pore has a significant impact on the pore water density, although the qualitative behavior is common for all channels. Increasing the hydrophilic character of the channel shifts the location of the extrema in the pore water density profile and the radial probability density. We have seen that the changes in the structural arrangement from/to the no-pass regime can be followed by means of a radial distribution function: the intensity and position of the second maxima in the RDF indicate the dependence of the confinement of water molecules on the pore radius and polarity.

\subsection{Permeability coefficients as function of the radius}

The thermodynamic properties presented in the previous section characterize the dependence of the pore occupancy and the water structure as a function of the pore radius and polarity. In addition to these static features, we now focus on how the dynamics of the permeating water molecules depend on channel radius and polarity. To describe the mobility of water molecules inside the channel we used the permeability coefficients introduced in section 2.2. The diffusive permeability $p_{d}$ was be computed by monitoring the total number of water molecules completely permeating the channel after a given lapse of time. Since the main role of biological water channels is to respond to gradient changes between different compartments, the osmotic permeability is a key quantity. In the previously chapter we have used a collective coordinate to derive $p_{f}$ from equilibrium simulations for completely filled single-file channels. However, to obtain the osmotic 
permeability coefficient using equilibrium simulations, for the current study we must choose a coordinate that can be applied to all permeation regimes, $i$. e., it must hold beyond the single-file configuration. To this end, we will use the collective coordinate, described in the Theory and Methods chapter, section 2.2.2, as the time dependent cumulative displacements of the water molecules in the channel, normalized to the channel length. Since the definition of the collective coordinate does not rely on single-file transport, it is applicable to water molecules permeating pores of any radius. See the pg. 21 for a detailed derivation of the osmotic permeability coefficient using this collective coordinate..

In this study, the pore radius is the only parameter was varied given a channel polarity, and therefore is the independent variable. However, we have seen that the pore water occupancy and the pore water density, are useful quantities to describe the system. In the following we will therefore discuss the relationship between the permeability coefficients, the radius of the pore and the average pore occupancy.

In figure 5.6, panel a and b show the osmotic $\left(p_{f}\right)$ and diffusive $\left(p_{d}\right)$ permeability coefficients as function of the pore radius for different pore polarities. Panel a' and b' show $p_{f}$ and $p_{d}$ as function of the pore occupancy per nm. The rough global dependence of the permeability coefficients on the radius requires at least a polynomial of second order to be described, although close examination reveals severe deviations (data not shown). The slope change of the osmotic permeability as a function of the radius indicates transition between permeation regimes with changing radial dependence. Figure 5.7 shows the same information as Fig. 5.6 panels a and b, but the logarithmic scale clearly reveals the changes in slope. For $p_{f}$ there are three distinguishable regions of almost constant slope, which could all be well approximated by an exponential increase. Regrettably, there is no universal expression for the dependence of $p_{f}$ with the radius that accounts for all regimes. Equation 4.1 and 4.2, are only valid for a single-file, and even in that case there is no expression relating $D_{w}^{0}$ to the radius of the pore. The same holds for $p_{d}$ : relationships were derived for single-file and for macroscopic pores [5], but there are no general expressions for any value of $R$. For a pore of macroscopic dimensions one can apply Poiseuille's law to estimate the osmotic permeation coefficient [5],

$$
p_{f}=\frac{\pi R^{4} N_{A} k_{B} T}{8 L \eta \nu_{w}}
$$




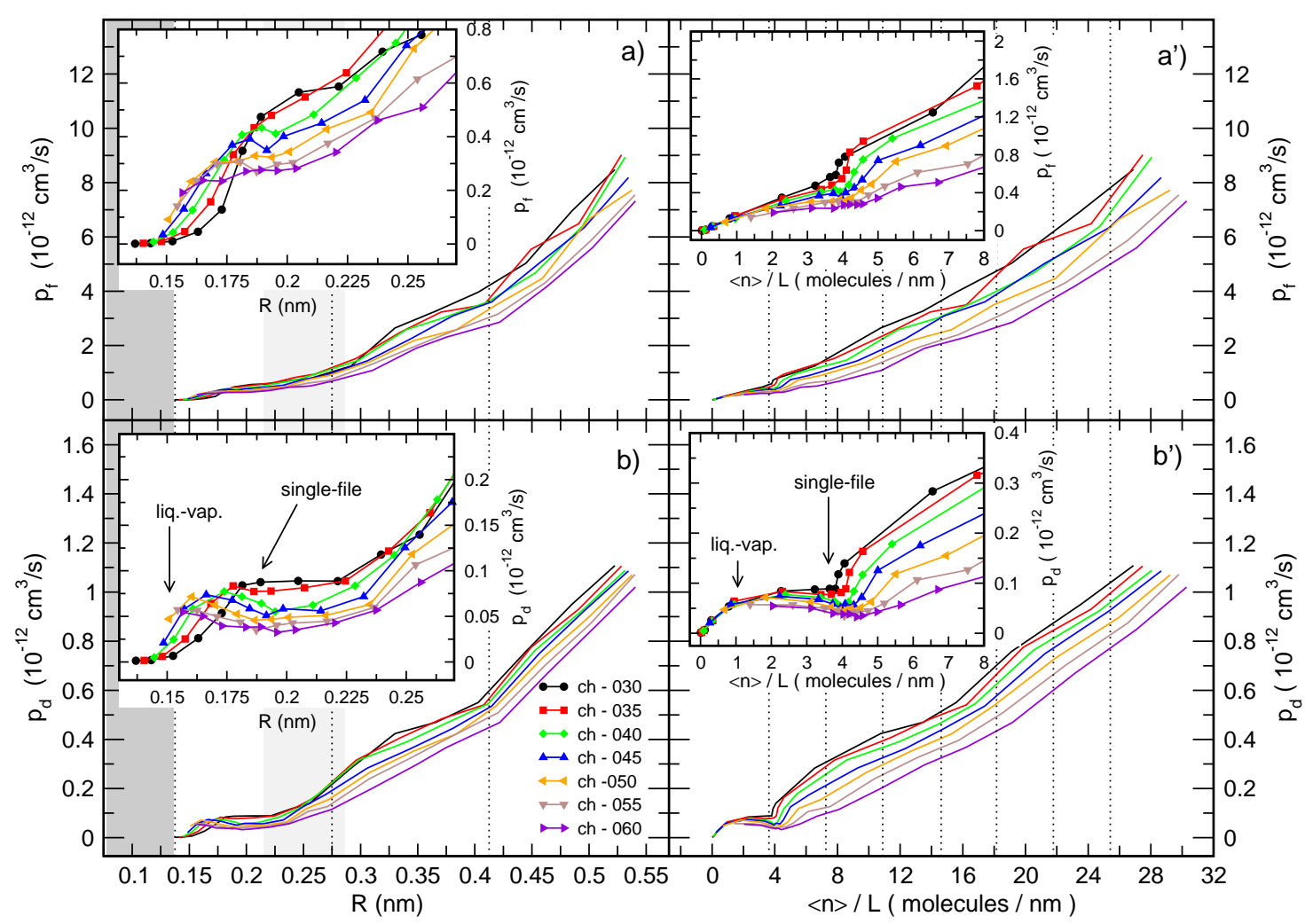

Figure 5.6.: a) and b) Osmotic (upper left panel) and diffusive (lower left panel) permeability coefficients for different pore polarities as a function of the pore radius. a') and b') display the osmotic (upper right panel) and diffusive (lower right panel) permeability coefficients as a function of the pore water occupancy (divided by the length of the pore). The insets are detailed views of the permeability coefficients at small pore radii. The dark gray area indicates a radius smaller than the water molecule radius, and the light gray area is positioned at the transition from/to the single-file regime. The vertical dashed lines indicate radii corresponding to multiples of a water molecule radius for a) and b), and multiples of 3.63 water molecules per $\mathrm{nm}$ in a') and b'). Error bars are not drawn for clarity, the uncertainty is below $10 \%$ of the permeability coefficient reported.

where $\eta$ is the viscosity of water. This expression has been tested correct for pores as small as $15 \mathrm{~nm}$ [166], but there is no physical justification for its applicability to pores of molecular dimensions, were the transport is diffusive and not convective [5]. Nevertheless, early molecular dynamics simulations of a $0.32 \mathrm{~nm}$ pore [57] reported relatively good agreement of the effective radius computed from equation 5.2 and the actual radius of this pore. Actually, in our simulations the $p_{f}$ at a radius beyond $0.4 \mathrm{~nm}$ can be well described by a $R^{4}$ functional form, although larger radii should be considered to establish the significance of 
this observation.

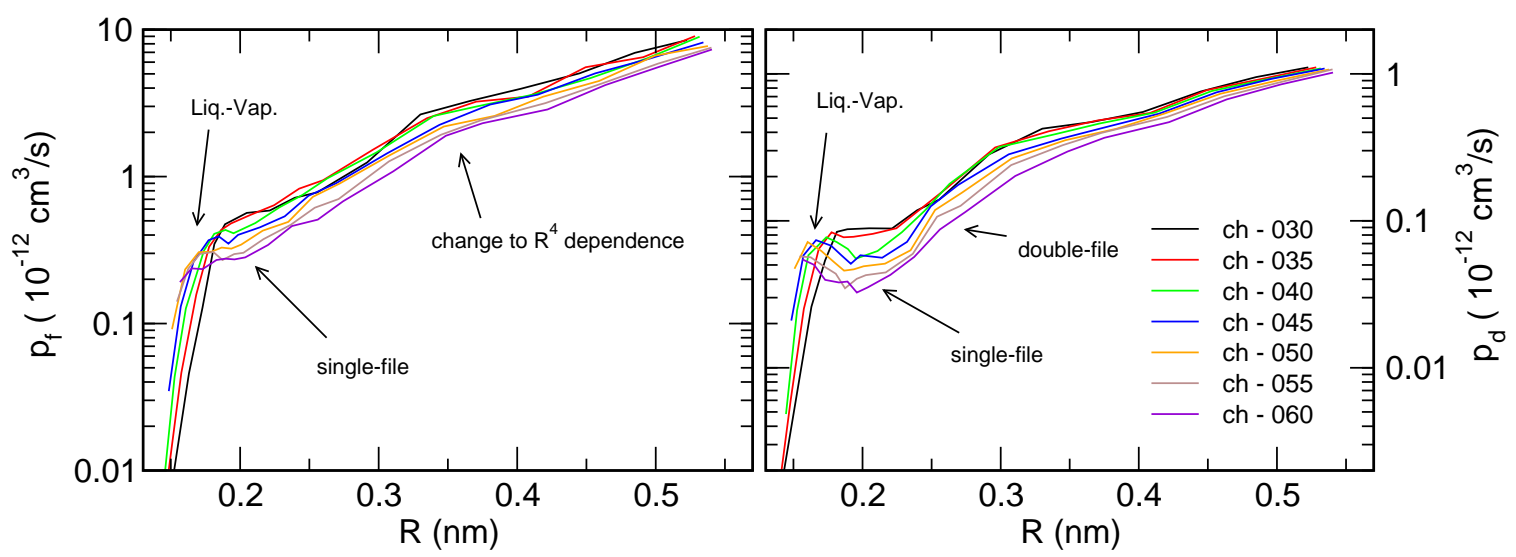

Figure 5.7.: Osmotic and diffusive permeability coefficients as function of the pore radius. The logarithmic scales reveals three different regimes for radius dependence of $p_{f}$, indicated by the change in slope. The change to $R^{4}$ dependence is also visible at $\sim 0.4 \mathrm{~nm}$. Before the single-file regime is fully established, at radius of $0.17 \mathrm{~nm}$, the thermodynamic state of the pore is characterized by liquid-vapor oscillations [32].

The mobility inside the channels with almost any radius larger than $0.2 \mathrm{~nm}$ is higher in the more hydrophobic channels. Below this radius, the permeability coefficients follow a behavior similar to the polarity dependence in the polyalanine channels (radius $\sim 0.16 \mathrm{~nm}$ ) described in section 4.2 .1 : low polarity pores are characterized by low permeability coefficients due to the incomplete pore occupancy, and high polarity pores have low permeabilities due to the friction caused by the attraction to the channel. The permeability coefficients at $\sim 0.16 \mathrm{~nm}$ radius are in the same range $\left(p_{f} \sim 1.5 \cdot 10^{-13} \mathrm{~cm}^{3} / \mathrm{s}\right)$ as the peptidic polyalanine channels. Once the low polarity pores are completely filled with water molecules, at radii larger than $0.2 \mathrm{~nm}$, their permeability coefficients are larger than the ones of the more polar channels, due to the larger water-pore interaction.

The osmotic permeability coefficients monotonically increase with the water pore occupancy and the radius. The channels of low hydrophilicity display an almost linear increase of $p_{f}$ with the occupancy until the configuration of maximally packed single-file is reached. The slope of the linear dependence of the $p_{f}(N)$ curve is below one. This implies that, since the average occupancy reflects opening times, the increased frequency of open states lowers the rate at which the molecules permeate under an osmotic gradient (otherwise the slope of $p_{f}(N)$ would have been one or larger). Nevertheless, since the channel is in the open 
state for longer periods, the overall osmotic permeability increases. Therefore, the regime connected to the liquid-vapor oscillations (burst-like behavior) does not necessarily imply fast permeations, as has been suggested [167]. At the highest density of the single-file there is a sudden increase of the osmotic permeability as function of the radius in a very small range of pore occupancy. This effect is due to the steady expansion of available area with the radius in the transition from single-file configuration. Here as well, the transition of the osmotic permeability becomes smoother for the more hydrophilic pores, and for ch-060 it is almost linear. At an occupancy of $\sim 5.6$ water molecules per $\mathrm{nm}$, corresponding to the midpoint between single and double-file, the fast increase of $p_{f}$ stops and remains growing linearly with the occupancy. As can be seen from Fig. 5.6 panel a', the consecutive crossings of occupancy regimes (single, double, triple, etc) increase the dependence of the $p_{f}$ on the occupancy. The limit is a $(\langle n\rangle / L)^{2}$ dependence, since the length-normalized occupancy in a macroscopic cylindrical pore is $\rho_{0} \pi R^{2}$, and we know that Poiseuille's law (eq. 5.2) has a $R^{4}$ dependence.

While the $p_{f}$ always increases with the radius, the diffusive permeability coefficient $p_{d}$ shows a remarkably different behavior, especially in the single-file regime. All channels show an increase of the $p_{d}$ with the pore occupancy at water pore densities lower than half the density of bulk water. In this pore range, the averaged occupancies reflect fractions of time in which the channels are open. Intermittent permeations of few water molecules contribute to the diffusive permeability, and therefore $p_{d}$ increases with the fraction of open time, i. e., with the radius. For channels more polar than ch-030 there is a drop in the $p_{d}$ for radii larger than the radius for which the single-file regime is welldefined. For ch-030 there is no decrease in the diffusive flux at this radius, but it remains almost constant. Once the radius of a hydrophilic channel is large enough to form a single-file configuration with a frequency higher than $50 \%$, the $p_{d}$ starts decreasing when increasing the radius further. The low diffusion constant of the single file regime with respect to the bulk water is a characteristic behavior [125, 164, 168]. The results of this work show that the decay of the diffusive permeability with the radius is characteristic of hydrophilic pores before the single-file configuration is reached. In the simulations presented by Beckstein et al. [155], the reported equilibrium diffusive flux as a function of the pore radius in a completely hydrophobic pore presents no reduction of diffusive flux, 
in agreement with our results and interpretations. At a pore radius larger than $\sim 0.2 \mathrm{~nm}$ the pore density decreases significantly and the water diffusivity starts increasing due to the larger volume available. To a lesser extend, the attenuation of $p_{d}$ is also visible at the radius corresponding to the establishment of the triplefile regime $(\sim 0.32 \mathrm{~nm})$, a local maximum for the less polar channels. The larger pores, beyond $0.4 \mathrm{~nm}$ display a $R^{2}$ dependence of $p_{f}$ with the radius. Since the diffusion constant approaches a constant value as the channels becomes wider (the bulk water diffusion constant, $D_{w}$ ), the dependence of $p_{d}$ with the radius approaches the macroscopic limit, $p_{d}=\pi R^{2} D_{w} / L[5]$.

To further rationalize the decay of the diffusive flux with the radius in the hydrophilic pores, we will consider the distribution of pore occupancies over the simulation time, see Fig. 5.8. Similarly to what was observed in section 4.2.1, the polarity of the pore has a big influence on the development of the occupancy distribution. For pores with a water density less than half the bulk density, corresponding to an average occupancy of 1.8 water molecules per nm, the pore occupancies are normally distributed around zero occupancy, and the behavior of water molecules in the pores is characterized by so-called liquidvapor oscillations [32]. Water molecules permeating the channel do so in small clusters, and the averaged occupancy reflects fractions of time in which the pore is open, $i$. e., proportional to $p_{d}$. The diffusive permeability of ch-030 does not decrease with increasing radius because, even at the highest density, the channel is still characterized by a significant contribution from empty states. After the averaged density reaches $0.5 \rho_{0}$, the dominant contribution is from the single-file, and therefore the diffusion permeability suffers a decrease due to the lower diffusivity of the single-file configuration as compared to individual water molecules or small clusters $[125,164,168]$. Highly hydrophobic channels do not show the decrease in the diffusive permeability since the liquid state is only stable at large radii, where the single-file configuration does not contribute significantly.

\subsection{The breakdown of proportionality between $\langle\mathbf{n}\rangle$ and $\mathbf{p}_{\mathrm{f}} / \mathbf{p}_{\mathrm{d}}$}

One of the most celebrated equalities in the framework of single-file transport is the ratio of osmotic and diffusive permeability, equation 2.25. As we already 


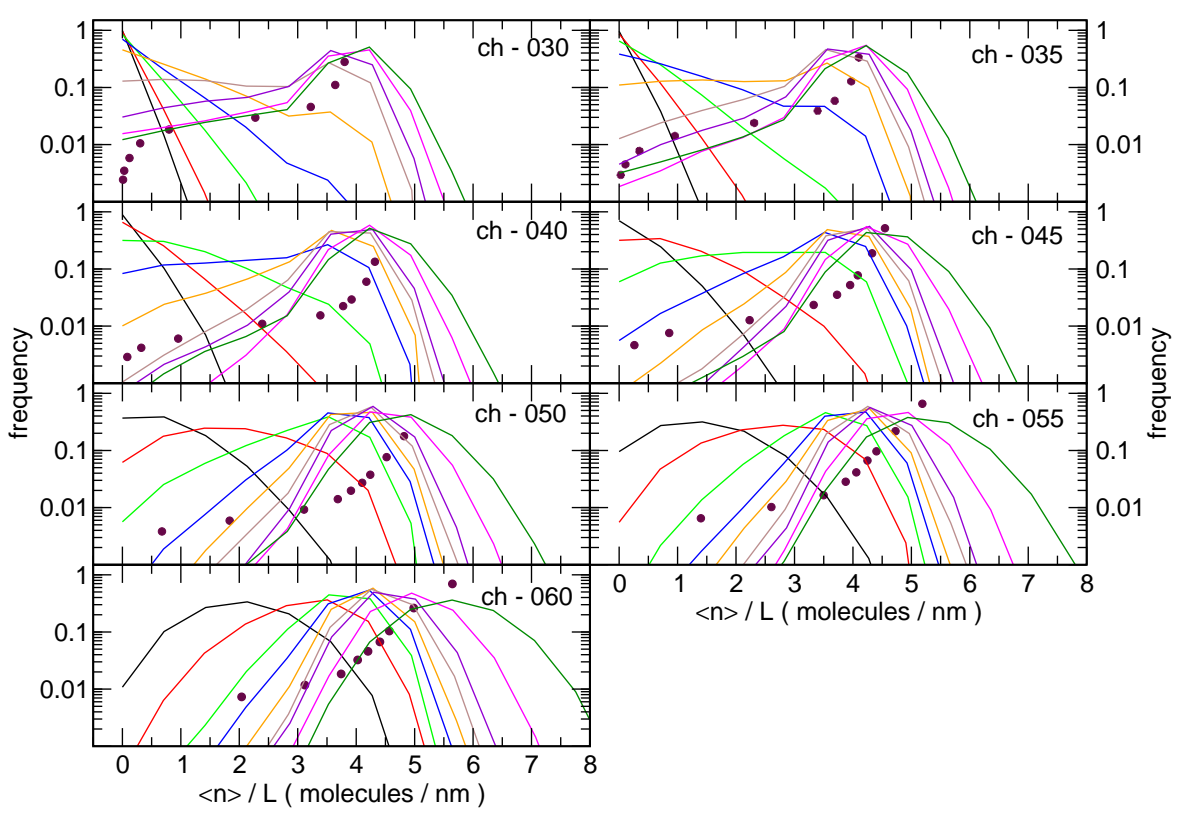

Figure 5.8.: Histograms of water pore occupancies for radius lower than $0.28 \mathrm{~nm}$ and different pore polarities in logarithmic scale. The line color is a visual guide to follow the different pore curves, which correspond to increasingly larger pore radii from left to right. Since the radius of the pores varies slightly between channel polarities, its value is not reported here for clarity. The filled dots indicate the averaged pore occupancy for each curve.

discussed in section 4.2.1, a generalized expression accounts for the effects of average time occupancies on the permeability coefficients in the single-file regime, or rather, under the no-pass condition. The generalized expression,

$$
p_{f} / p_{d}=\langle n\rangle+1
$$

was found to hold for a wide range of pore lengths and pore polarities, see Sec. 4.2.1. Here we will expand the study of the validity of equation 5.3, especially in the transition from the single-file to double-file regime, and we will characterize the ratio of permeability coefficients beyond the no-pass condition. Figure 5.9 shows a combined representation of averaged pore water occupancies and the ratio of permeability coefficients as a function of the pore radius.

The results presented in Fig 5.9 are in good agreement with equation 5.3 in the whole range of radii in the single-file region. The two curves drift apart at values 


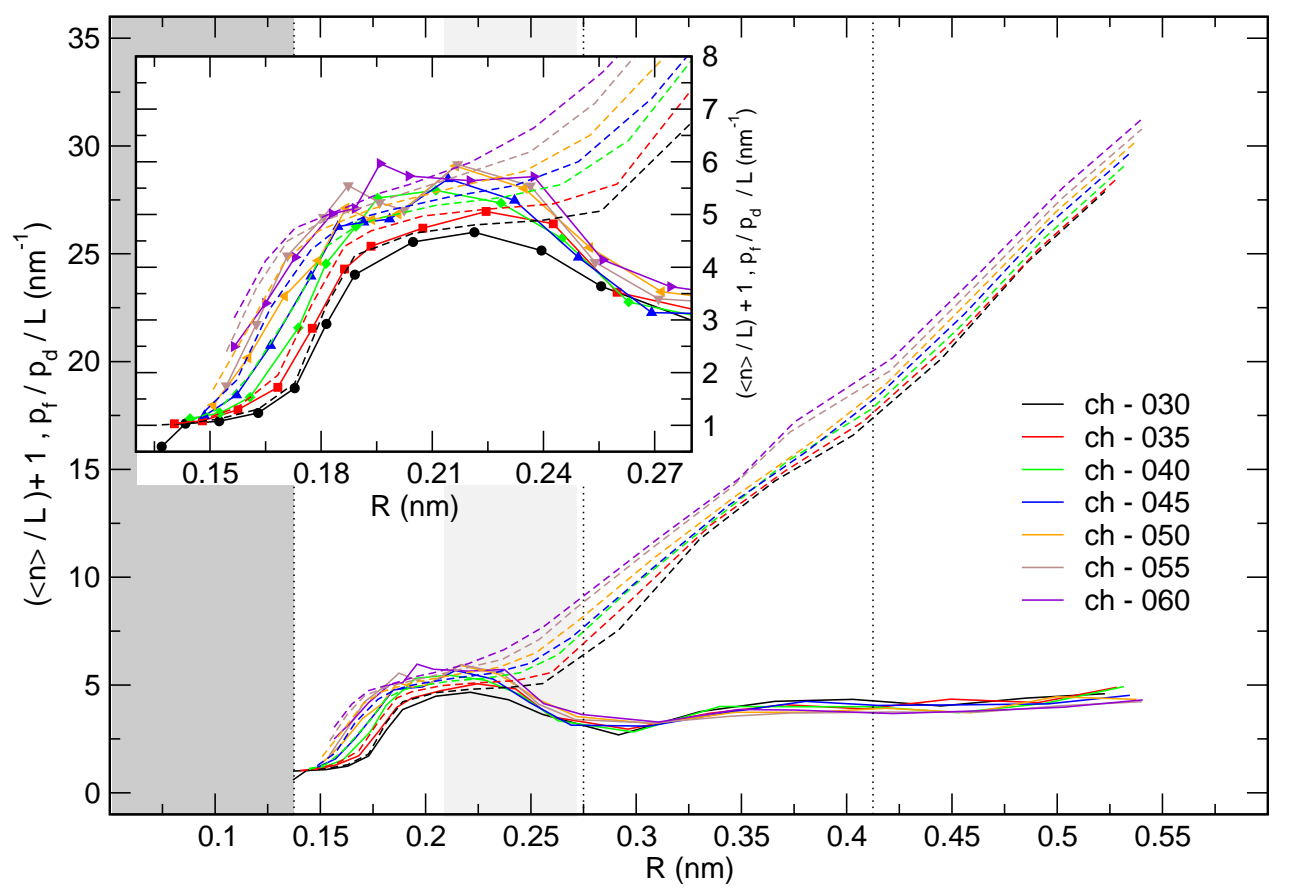

Figure 5.9.: Ratio of osmotic permeability coefficients, $p_{f} / p_{d}$ (solid lines), and averaged pore water occupancy plus one (dashed lines) as function of pore radius. Both dependent variables were normalized by the length of the pore. The inset focuses on the range of radius smaller than the diameter of a water molecule. The dark gray area indicates radius smaller the radius of a water molecules, and the light gray area indicates the transition involving the single-file regime.

of the radius that correlate with the structural or thermodynamic indicators used in this work, the local minimum in the water pore density as function of the radius and the second maximum in the RDF. In this sense, the ratio of $p_{f}$ and $p_{d}$ is a indicator of the transition from the single-file regime. Figure 5.10 displays the correlation between the averaged pore occupancy and $p_{f} / p_{d}$. The loss of single-file character is clearly indicated by the deviation from the straight line.

While the significance of $p_{f} / p_{d}$ is clear in the single-file transport, its value beyond that regime is not known. More precisely, it is not known which is the dependence with the radius in the interval between single-file and macroscopic pore [5]. In macroscopic pores, the osmotic permeability is described by equation 5.2 , and has an $R^{4}$ dependence. The diffusive permeability in macroscopic pores is derived by means of Fick's diffusion equation, and equals to $p_{d}=\pi R^{2} D_{w} / L[5]$. 
Figure 5.10:

Correlation between the ratio of osmotic and diffusive permeability and $\langle n\rangle+1$, both normalized by the pore length. The dashed black line indicates the expected result in the single-file regime according to equation 5.3 .

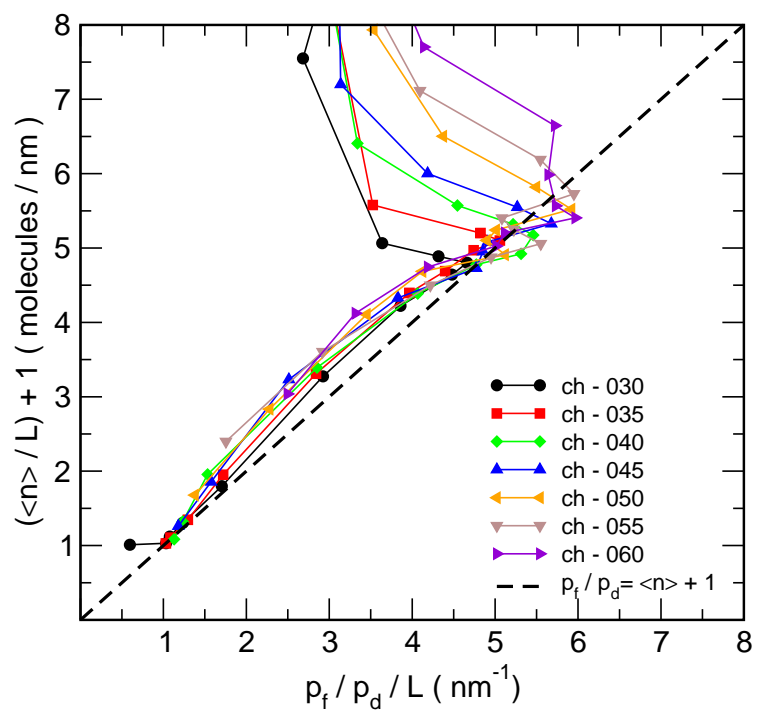

Therefore, for a macroscopic pore the ratio equals to

$$
\frac{p_{f}}{p_{d}}=\frac{R^{2} N_{A} k_{B} T}{8 D_{w} \eta \nu_{w}}
$$

Although the results we obtained for the permeability coefficients for large pore radii hinted at convergence towards the radial dependence of a macroscopic pore, the ratio of permeabilities is still far from the expected macroscopic behavior. Rather than increasing with the radius of the pore, the ratio decays to a local minimum after the single-file regime is left. We have seen in secticon 2.2.2 that a useful mechanistic picture of the single-file transport considers a $p_{f}$ event as a concerted hop of the water molecules between states equivalent by symmetry, and a $p_{d}$ event occurs after a complete permeation of a water molecule. A value of $\left(p_{f} / p_{d}-1\right)$ below the averaged occupancy indicates more hops of the water molecules column than needed to completely translocate a water molecule. In agreement with this interpretation, the decay in the $p_{f} / p_{d}$ quotient correlates with the minima in the density: water molecules can easily diffuse perpendicular to the pore axis, and transient disruptions of the water chain allow motions along the pore axis that do not result in a net transport of a water molecule. After reaching a radius of $0.35 \mathrm{~nm}$, the ratio remains almost constant until $\sim 0.45 \mathrm{~nm}$. At that point, there seems to be a general trend towards higher values, possibly converging to the $R^{2}$ dependence. To clearly resolve if the ratio approaches the macroscopic limit, wider channels should be studied. 
Dividing the averaged pore occupancy by $\left(p_{f} / p_{d}-1\right)$ clearly yields one in the single-file regime. Due to the $R^{2}$ dependence both in the average water occupancy and the $p_{f} / p_{d}$ ratio, for a macroscopic pore the result is expected to be constant, namely $8 D_{w} \eta \nu_{w} / R T$. If the permeation mainly occurs via independent singlefile like structures embedded in the channel, the ratio is expected to be directly proportional to the averaged occupancy at the single-file regime. Figure 5.11 panel a shows the value of $(\langle\mathrm{n}\rangle+1) /\left(p_{f} / p_{d}\right)$ as function of the radius. The single-file regime is characterized by of one, and the potential changes of file regimes are indicated by crossings of integer values of the abscissa.

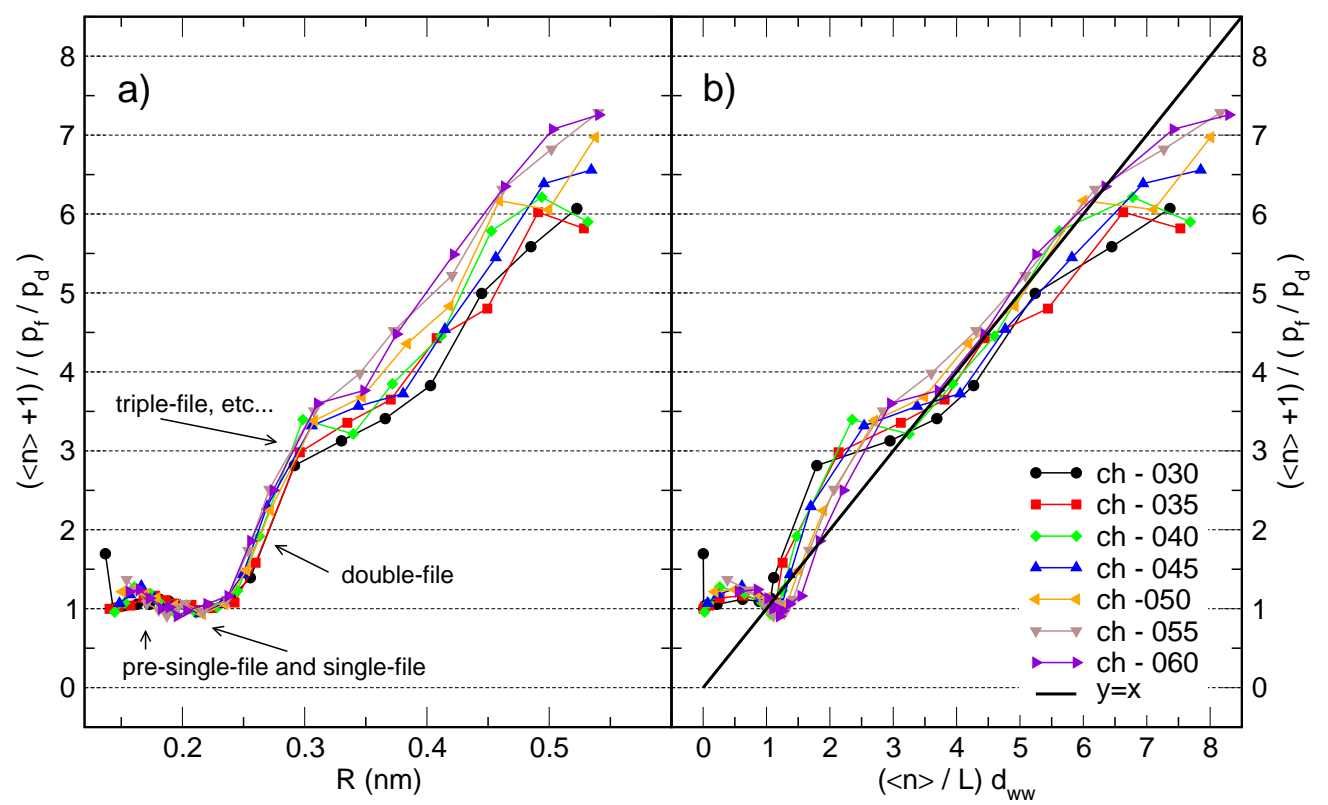

Figure 5.11.: a) Ratio of $(\langle n\rangle+1)$ to $\left(p_{f} / p_{d}\right)$ as function of the radius. b) Correlation between $(\langle n\rangle+1) /\left(p_{f} / p_{d}\right)$ and $(\langle n\rangle / L) d_{w w}$, where $d_{w w}$ is the typical water-water distance, established to $0.275 \mathrm{~nm}$. Disregarding the radius where the pore presents a local minima in the water density and radii larger than $0.45 \mathrm{~nm}$, the ratio of occupancies and permeability coefficients correctly identifies the transitions between different file regimes. The solid black line indicates $(\langle n\rangle / L) d_{w w}=(\langle n\rangle+1) /\left(p_{f} / p_{d}\right)$.

Panel b in Fig. 5.11 shows the correlation between the computed $(\langle\mathrm{n}\rangle+$ $1) /\left(p_{f} / p_{d}\right)$ and the expected value based on the averaged occupancies. To determine the number of single-file columns per nm, the typical water-water distance is used $(0.275 \mathrm{~nm})$. The agreement between $(\langle\mathrm{n}\rangle+1) /\left(p_{f} / p_{d}\right)$ and $(\langle\mathrm{n}\rangle / L) d_{w w}$ is remarkably good: deviations occur at the occupancies that correspond to the minima in the water pore density and in the range of radius where the overall 
behavior presumably approaches the macroscopic laws of hydrodynamics. Since only the displacements along the pore axis contribute to the diffusive permeability, this result indicates that the relevant motions along the pore main axis effectively take place within single-files.

\subsection{Summary and conclusions}

In this chapter, we presented a detailed study of the influence of the radius and the polarity of narrow hydrophilic pores on the water permeation. The structural and dynamical information obtained complements and expands the knowledge of the structure-activity relationship in water channels. Such knowledge is valuable to understand the permeation mechanism of naturally occurring channels and to design channels of desired properties. The presented results are useful to predict the response in the channel flux to changes in the concentration gradient between the solutions at each end of the channel, or which radius and polarity would allow a fast flux of water molecules while preserving the single-file regime. Since single-file channels are generally a requirement for the selectivity towards different solutes, we therefore paid special attention to this permeation regime.

The analysis of the pore water density revealed characteristic oscillations in channels of radii smaller than three times the radius of a water molecule. Prior to the establishment of an uninterrupted flow of water molecules, on average at a radius of $\sim 0.17 \mathrm{~nm}$, the density fluctuations can be well described by a liquidvapor equilibrium [32]. A perfectly packed single-file configuration presents a local maximum in the pore water density as a function of the pore radius, followed by a local minimum indicating the transition to a double-file regime. The polarity of the channel scales the water pore density: an increase in hydrophilicity of the pore leads to a corresponding increase of the pore water density, and modulates at which radius the transitions between different regimes takes place. At large pore radii, all water pore densities converge to the bulk density, and therefore the pore occupancy directly scales with the area and length of the pore.

The permeability coefficients, both osmotic and diffusive, were characterized as function of the pore radius and the averaged occupancy. In the single-file regime, the osmotic permeability always increases with the pore radius, scaling linearly with the pore occupancy until the densest single-file configuration is achieved. 
On the contrary, the diffusive permeability experiences a local maximum at radii where the density fluctuations average to half the bulk water density, and falls to a local minimum at the densest single-file configuration. Along with the transition from single-file to double-file, the permeability coefficients increase with the radius. Both coefficients show a gradual increase in the slope depending on the radius, converging to the macroscopic radial dependence for large radii.

The ratio of osmotic and diffusive permeability coefficients was found to equal the averaged pore occupancy (plus one) for all pore polarities and radii that guarantee a no-pass condition. Beyond this single-file arrangement, the value of $\left(p_{f} / p_{d}-1\right)$ remains almost constant, equal to the occupancy corresponding to the densely packed single-file configuration. This implies that the confinement of the water molecules preserves the collective motions of water chains, effectively acting like single-files in parallel. As the channel becomes much wider, the ratios converge towards their expected macroscopic radial dependence.

For the design of new water channels, we have seen that the radius and polarity play a major role in determining the flow of water molecules, in contrast to the results presented in the previous chapter, where we showed that the length has almost no impact on the osmotic permeability. In general, if the selectivity of the pore is not an issue, large apolar channels are the most effective in conducting the water flux. If we are constrained to the narrow single-file regime, less hydrophilic channels allow the single-file to persist at a larger radius than highly polar channels, and with faster rates. 
5. Influence of the pore radius and polarity on the water permeability 


\section{Not only enthalpy: entropy dominates ion permeation barriers in single-file channels}

\section{Summary}

In previous chapters, we focused our study on the determinants of water permeability in narrow pores. Since the peptidic water channels presented before also function as cation channels, we will now concentrate on one of the multiple factors that influences ion permeability through single-file pores, and compare this with the determinants underlying water permeation. Here, we focused on the effect of channel length on the barrier for potassium ion permeation through single-file channels. Using series of peptidic gramicidin-like and simplified ring-structured channels, both embedded in model membranes, we obtained two distinct types of behavior: saturation of the central free energy barriers for peptidic channels and a linear increase in simplified ring structured channels with increasing channel length. The saturation of the central free energy barrier for the peptidic channels occurs at relatively short lengths, and it is correlated with the desolvation from the bulk water. Remarkably, decomposition of free energy barriers into enthalpic and entropic terms reveals an entropic cost for ion permeation. Furthermore, this entropic cost dominates the ion permeation free energy barrier, since the corresponding free energy contribution is higher than the enthalpic barrier. We conclude that the length dependence of the free energy is enthalpy dominated, but the entropy is the major contribution to the permeation barrier. The decrease in rotational water motion and the reduction of channel mobility are putative origins for the overall entropic penalty. The different length dependence of water and ion permeation opens up novel ways to steer the selectivity 


\section{Entropy reduction increases single-file ion permeation barriers}

of natural and model channels.

\subsection{Introduction}

Ion channels, located in lipid membranes that isolate organelles and cells, allow the passage of ions from one side of a membrane to the other. Their structure and composition is diverse, although they share some characteristics, such as hydrophilic lumen composed of peptide backbone carbonyl groups and narrow selectivity filter [169]. Their function affects electrochemical gradients, and thus ion channels play a fundamental role in energetics of living cells. A subtle balance of ion gradients is, for example, responsible for the polarization-depolarization of neurons that leads to electrical signal transmission in nerve cells [7]. Ion channels evolved to be selective [27], either to single ionic species like potassium [26, 170$173]$ or to a certain classes of ions, e.g. gramicidin only allows the passage of small univalent cations $[174,175]$.

Additionally, molecular channels play an essential role in nanotechnology. Accurate knowledge of their structure-activity relationship is prerequisite to design fine-tuned channels that permeate selectively, allow the detection of ligandprotein interactions $[49,176]$ or monitor chemical reactions [177].

To gain understanding in the relationship between ionic permeation characteristics and the physical determinants underlying permeation in ion channels, we carried out a systematic study using simplified model pores. Previous work thoroughly described the effects of channel radius, surface character and flexibility on the permeation of water and ions through model channels $[155,178]$. Here we focused on the effect of the channel length on the permeation of potassium ions across single-file channels.

An atomistic description of the system is required to understand the interactions involved in ion permeation. The use of molecular dynamics (MD) simulations together with an all-atom force-field establishes a balanced compromise between accuracy and sampling power. Nevertheless, due to the long time scales required to observe spontaneous passage of ions [7], direct calculations of ion fluxes are not feasible. The use of strong forces or large membrane potentials to impose ion permeations would introduce conditions that differ from experimental or physiological conditions [179]. Here, we will concentrate on the thermo- 
dynamic function that characterizes systems in equilibrium, i.e. the free energy. Since we are interested in the passage of ions across the main pore axis, we extract free energy profiles for ion permeation in the form of Potentials of Mean Force (PMF)[104] as means of comparing energetic costs for ion permeation. The PMF can be used as basis for coarse-grained theories of ion conductance [58, 180].

Electrostatic interactions are fundamental components of ion permeation. Therefore, methods such as continuum-electrostatic Poisson-Boltzmann calculations are routinely used to study the energetics of ion permeation. However, these methods may suffer from limitations because they do not explicitly account for the behavior of individual water molecules [156], and effects arising from channel flexibility [181, 182]. Here, we present free energy profiles from atomistic, explicit solvent MD simulations that take these effects explicitly into account. From these simulations we extracted the thermodynamic components of the free energy, the enthalpy and the entropy, to quantify their contribution to the resulting transport barrier.

\subsection{Theory and Methods}

Design and set up A series of $\mathrm{D}, \mathrm{L}$ polyalanine peptides with increasing length and a $\beta$-helix gramicidin A folding motif were used to study the energetics of potassium ion permeations in peptidic environments (Fig. 6.1a, 6.1a'). The modeling of the polyalanine peptides was done as previously described in chapter 4.1.2. In short, a monomer of the gramicidin A channel in the head-to-head helical conformation was used to construct shorter and longer channels. The template structure has all residues mutated to alanine in order to achieve high regularity among the channels. The mutated gramicidin monomer, p-15, was elongated or shortened in steps of 2 residues. The studied peptidic channels range from p-11 to p-31, which spans a length between 0.8 to $2.6 \mathrm{~nm}$. Soft position restraints $\left(500 \mathrm{~kJ} / \mathrm{mol} / \mathrm{nm}^{2}\right.$ harmonic force constant) on the peptide backbone and termini capping groups, formyl and ethanolamine, ensure the peptide to remain in the $\beta$-helix fold and a well defined access to the channel. To isolate the effect of the channel length, matching membranes were constructed using octane molecules (Fig. 6.1a"), also described in chapter 4.1.2. Unwanted deviations in the adjustment of the membrane-peptide interface can give rise to 
different contributions of the ion-solvent interaction to ion permeation. Since ion-water interactions are key energetic components, special care must be taken in adjusting the membrane thickness to ensure consistency over the studied systems. To guarantee a stable octane slab, we used pressure coupling only in the $x-y$ plane, normal to the channel main axis.

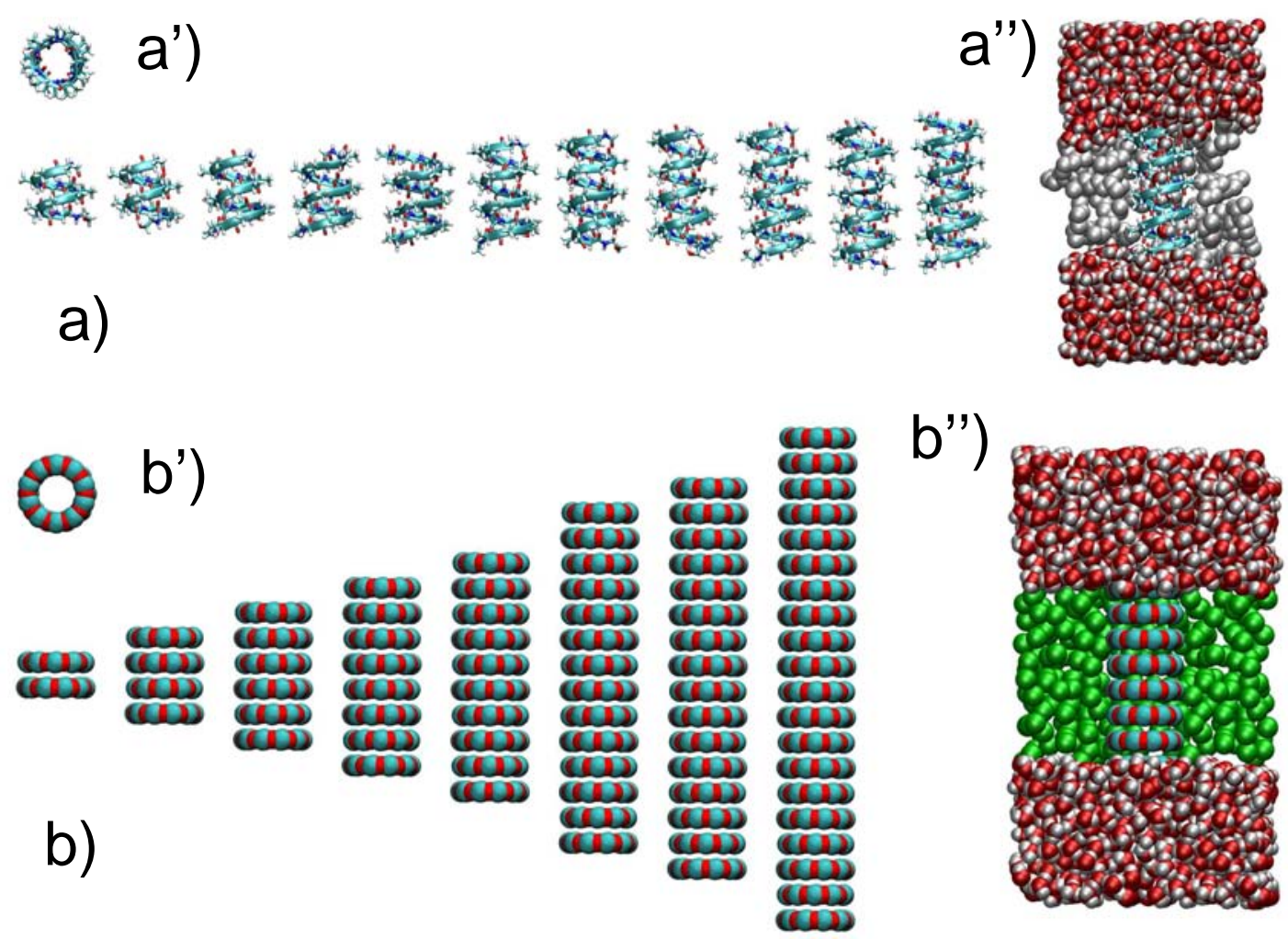

Figure 6.1.: Schematic representations of model channels used for the study. a) Side view of polyalanine pores of increasing number of residues, $p$-11 to p-29. a') Top view of p-17 channel. a") Side view of a typical simulation box for polyalanine channels. Half of the octane layer has been omitted for clarity. b) Side view of ring-structured channels of increasing number of rings. b') Top view of r-02. b") Simulation box for $r-08$, half the argon atoms were removed for clarity.

A second set of systems (ring-05), built of purely artificial ring structured channels was also studied (Fig. 6.1b, 6.1b'). The channel structure was inspired by a design of Crozier et al. [183], and it is reminiscent of the channels used to study the effect of the pore radius on the water permeability (Chap. 5.2). The rings, consisting of alternating carbon and oxygen atoms, were connected with bonds and were stacked to form a channel. Each ring was rotated with 
respect to the previous one to place carbons and oxygens on top of each other. The radius of the channels in the narrow regions was $0.18 \mathrm{~nm}$. The average inter-plane distance was centered at $0.32 \mathrm{~nm}$. Both the radius and inter-plane distances guarantee a single-file channel. The shortest ring channel contained two rings, r-02, was elongated in steps of two rings until r-20, spanning a length range of $\sim 0.5 \mathrm{~nm}$ to $\sim 7 \mathrm{~nm}$. All atoms in the channel were restrained by strong position restraints $\left(4000 \mathrm{~kJ} / \mathrm{mol} / \mathrm{nm}^{2}\right.$ harmonic force constant). An artificial membrane was constructed around the channel by position restraining LennardJones particles in their $x$ - $y$ plane (Fig 6.1b"), which was constructed normal to the channel main axis. This approach also guarantees a perfect match between the membrane thickness and the channel. We used constant volume simulations for these systems.

The same ring-structured channel set-up was used to study the effect of polarity on the free energy barriers for ion permeation. Charges were augmented by $-0.2 \mathrm{e}$ and $+0.2 \mathrm{e}$ for oxygen and carbon respectively, and the force constant for restraining the atom $z$ position was decreased from $4000 \mathrm{~kJ} / \mathrm{mol} / \mathrm{nm}^{2}$ to $500 \mathrm{~kJ} / \mathrm{mol} / \mathrm{nm}^{2}$. We refer to these systems as ring-07.

All the systems were solvated with $\sim 1200$ water molecules and $\mathrm{KCl}$ was added to achieve a $150 \mathrm{mM}$ concentration. All systems were energy minimized and simulated at $300 \mathrm{~K}$ for $20 \mathrm{~ns}$. The first $2 \mathrm{~ns}$ were discarded for equilibration.

Interactions between all atoms of the peptides, ring channels and the membrane were described by means of the OPLS all-atom (OPLSAA) force field [78, 79], and water molecules were described by the TIP4P model [80]. Potassium and chloride ion parameters were taken from Åqvist [184], and Chandrasekhar et al. [185], respectively. Parameters for oxygen and carbon atoms in the ringstructured channels ring-05 were assigned as peptide carbonyl atom types, taken from the OPLS/AA force field. The charges were increased from $\pm 0.5 \mathrm{e}$ to \pm 0.7 e for the ring-07 channels, as previously explained. All simulations were performed using the GROMACS 3.3.1 simulation software [134, 135]. Electrostatic interactions were calculated at every step with the particle-mesh Ewald method [93, 136]. Short-range repulsive and attractive dispersion interactions were simultaneously described by a Lennard-Jones potential, which was cut off at $1.0 \mathrm{~nm}$. The SETTLE [83] algorithm was used to constrain bond lengths and angles of water molecules, and LINCS [82] was used for all other bonds, allowing 
a time step of $2 \mathrm{fs}$. The temperature was kept constant by weakly coupling the system to an external heat bath [95] (time constant $\tau=0.1 \mathrm{ps).} \mathrm{For} \mathrm{the} \mathrm{constant}$ pressure simulations a weak semi-isotropic coupling to a pressure bath of $1 \mathrm{~atm}$ was applied $(\tau=1 \mathrm{ps})$.

Computing PMF for ion permeation Umbrella sampling (US) [112] simulations were introduced in the section 2.3.1 as a robust methodology to compute free energies. In this work, we applied US to obtain biased position histograms along the ion reaction coordinate. The reaction coordinate was defined as the principal axis of the channel, constructed to be the $z$ axis of the simulation box. The reaction coordinate was divided in equidistant windows separated by $0.01 \mathrm{~nm}$. To select starting configurations for the US, the equilibrium trajectories of $20 \mathrm{~ns}$ were used. For each window, a water molecule in the nearest vicinity of the target position was selected and exchanged by a potassium ion. A chloride ion was added in the solution to neutralize the system. After energy minimization, the system was ready for the production run.

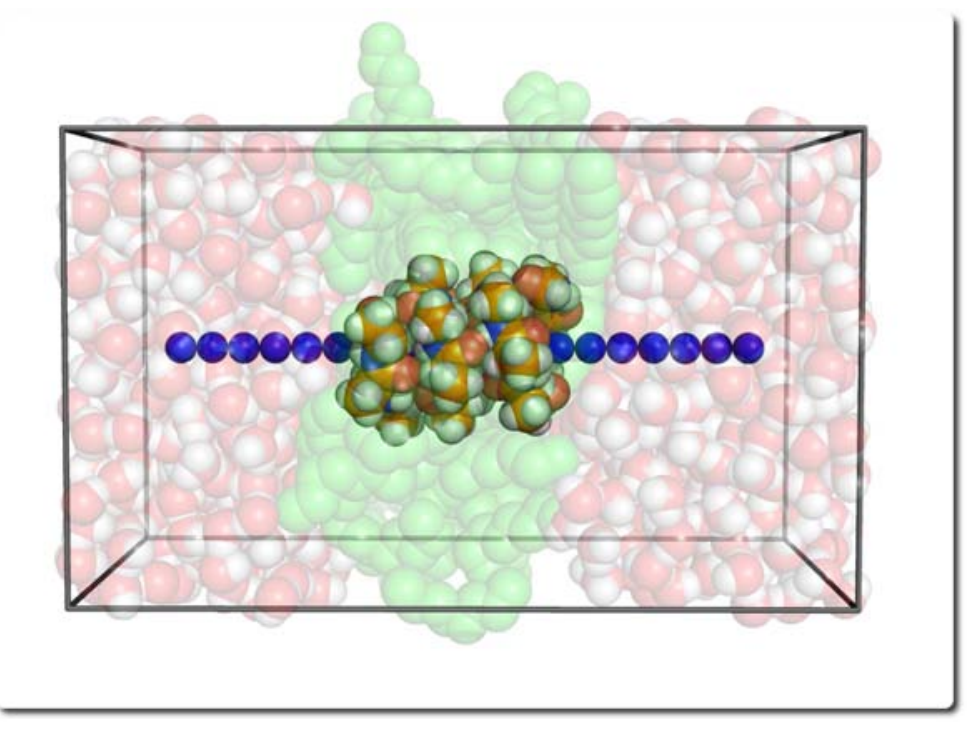

Figure 6.2.: Illustration of the reaction coordinate chosen for the umbrella sampling simulations. The blue balls indicate the position of the potassium ions. Only a tiny fraction of all positions used as reference position for the biasing potential are shown. The membrane is indicated by a green slab of octane molecules.

During the umbrella simulation, a harmonic potential of $4000 \mathrm{~kJ} / \mathrm{mol} / \mathrm{nm}^{2}$ was applied on the ion along the reaction coordinate. To ensure complete sampling 
on the ion's plane normal to the reaction coordinate, and a well defined concentration of channels, a cylindrical potential was implemented. A repulsive force $F(r)=-k_{c}\left(r-r_{c}\right) H\left(r-r_{c}\right)$ on the ion, normal to the reaction coordinate, restrained the ion to a cylinder of radius $r_{c}=0.5 \mathrm{~nm}$, whose axis was centered along the pore. Here, $r$ denotes the distance from the cylinder axis, $k_{c}=400 \mathrm{~kJ} / \mathrm{mol} / \mathrm{nm}^{2}$ the force constant, and $H$ the Heaviside step function.

Each umbrella window was simulated for 600 ps in its corresponding ensemble (NPT for polyalanine channels and NVT for ring structured channels). For the analysis, the first $100 \mathrm{ps}$ were discarded as equilibration. To recover the unbiased position probability distribution, the weighted histogram analysis method (WHAM) [122] was employed. The WHAM equations were iterated until a tolerance of $10^{-6}$ for the unbiased probability distribution was achieved. To account for the periodicity of the system, a cyclic version of WHAM was implemented. The PMF was obtained from the unbiased position probability distribution $p(z)$, as $-k_{B} T \ln p(z)$.

Statistical errors of the PMF profile were computed using bootstrap analysis. From $N$ histograms available, $N$ histograms were randomly selected (allowing duplication) and used in a subsequent WHAM procedure. We constrained the random selection of histograms to sample the complete reaction coordinate, avoiding gaps. We considered only complete histograms as independent data points, yielding a rather conservative estimation for the uncertainty. Bootstrapping was done 50 times yielding 50 PMFs for each channel. From the 50 PMFs, standard deviations were calculated. The uncertainty was generally $\leq 1.5 \mathrm{~kJ} / \mathrm{mol}$.

Decomposing PMF features in polyalanine channels The PMFs for ion permeation through polyalanine channels can be separated in contributions from an entrance barrier and a central barrier which increases with channel length (Fig. 6.3).

Each PMF for the polyalanine channels was decomposed in three overlapping Gaussians. To reduce the number of fitting parameters, p-29 was used to extract the height of the Gaussian located at the entrance barrier. This was done by a free fit (9 parameters) of three Gaussians, centered around the channel lumen and channel entrances. The height of the fitted Gaussian corresponding to the pore entrance for p-29 was then used as a constraint in the multi-Gaussian fit 


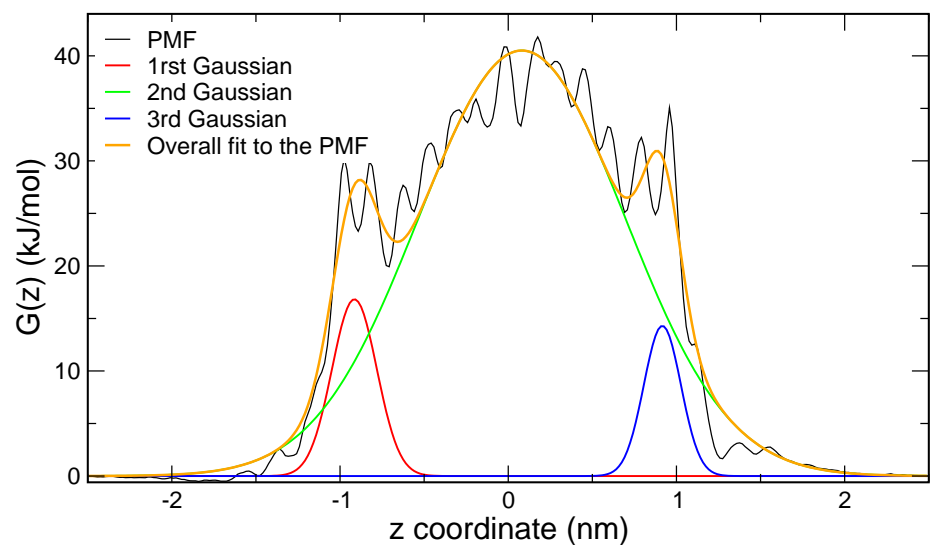

Figure 6.3.: Fitting of 3 Gaussians on the PMF. The orange curve is the resulting overall fit, decomposed in three functions (red, green and blue curves). In the free fit all 9 parameters were determined using a least square fit to the PMF.

for the rest of the polyalanine PMFs. From these constrained fits, the height of the central barrier was obtained as the height of the central Gaussian.

Entropic and enthalpic contributions to the free energy To gain insight into the thermodynamic nature of ion permeation barriers in the model channels, we extracted the enthalpic and entropic components of the free energy barriers for ion permeation (Fig. 6.6, 6.8). In contrast to the wealth of techniques to obtain free energies [108, 186, 187], the number of methods for calculating enthalpic and entropic contributions of a fully hydrated system is rather limited.

A direct calculation of the enthalpic penalty for ion permeation is complicated by the large energy fluctuations intrinsic in Molecular Dynamics simulations of thousands of particles. Nevertheless, one can compute time averaged potential energies for defined configurations of the system of interest. In this work, only the polyalanine channels rendered statistically converged estimates for the enthalpy by this approach. Enthalpy contributions were extracted by harmonically restraining the ion in the highest energetic position of the PMF and in the bulk. The ion in the bulk was fixed $1.4 \mathrm{~nm}$ from the pore entrance for all channels. For each channel and ion position, 5 simulations of 10 ns were performed under the same simulation conditions as in the US runs. After discarding the first $1 \mathrm{~ns}$, the enthalpy of the system in that particular ion configuration was computed as the sum of the potential energy and the $p V$ term (where $p$ is the pressure in the sys- 
tem and $V$ is the volume), corresponding to the enthalpy in an isothermal-isobaric ensemble. The contribution from the position restraint energy was removed from the estimate. The enthalpy difference for each channel, $\Delta H$, was computed as $H_{\mathrm{K}^{+} \text {pore }}-H_{\mathrm{K}^{+} \text {bulk }}$, using averaged values from the 10 independent simulations.

To compute entropy differences, several strategies [188, 189] have been proposed to circumvent the sampling problem of direct entropy calculation. Regrettably, methods that compute $\Delta S$ via thermodynamic integration converged too slowly for our systems (data not shown).

Instead, $\Delta S$ was computed via the temperature derivative of the free energy barrier at constant pressure and number of particles $N$,

$$
\Delta S=-\left(\frac{\partial \Delta G}{\partial T}\right)_{N, p}
$$

The former expression is exact, but requires a partial derivative to be evaluated. The partial derivative was approximated by the total derivative, $(d \Delta G / d T)_{N, p}$, assuming a constant heat capacity difference in the simulated temperature range, $(\partial \Delta H / \partial T)_{N, p} \approx 0$. The validity of this approximation was checked by computing $\Delta H$ and $\Delta G$ independently. If the difference of these two values equals the $-T \Delta S$ result obtained by means of equation 6.1 , it implies that the approximation of constant heat capacity difference to compute $\Delta S$ is justified.

PMFs were computed at temperatures ranging from $280 \mathrm{~K}$ to $320 \mathrm{~K}$ in steps of $10 \mathrm{~K}$ using US simulations as described earlier. This implies 4 additional US simulations for each studied channel. To reduce the computational effort, only 4 representative channels of the polyalanine and 4 of the ring-structured series were analyzed. From $\Delta G(T), \Delta S$ was computed by a weighted linear least-squares fit. The weights were chosen according to the statistical uncertainty derived by bootstrap analysis of the PMFs. The statistical error for the entropy estimate was extracted from the uncertainty in the slope of the fitted line. The correlation coefficient was generally $\sim 0.9$.

To get qualitative insight into individual contributions to the overall entropy barrier for ion permeation, we extracted the rotational entropy of water molecules in the channel and the conformational channel entropies. To a first approximation, the entropic contribution due to rotation of water molecules inside the pore was estimated by considering the position-dependent rotational probability 
distribution, $\rho_{\text {rot }}(z)$, of $n$ independent water molecules inside the channel. Details on the calculation of this probability distribution are given in Appendix A. From $\rho_{\text {rot }}(z)$, the rotational entropy was computed along the pore length $\mathrm{L}$ as $S_{\text {rot }}=-k_{B} \int_{L} d z \rho_{\text {rot }}(z) \ln \rho_{\text {rot }}(z)$. To compute the entropy cost of placing an ion at the center of the channel, a system without the ion in the channel was used as the reference state. We used the Schlitter [190] formula and the quasi-harmonic (QH) approximation of Andricioaei and Karplus [191] to estimate the channel entropy based on atomic fluctuations. Both represent an upper bound for the entropy, but they serve to gain a qualitative picture.

To evaluate these rotational and conformational entropies, estimates for eight representative channels (p-15, p-19, p-25 and p-29 for the polyalanine channels; $\mathrm{r}-04, \mathrm{r}-08, \mathrm{r}-14$ and $\mathrm{r}-20$ for the ring-05 systems) were obtained from 2 simulations of $100 \mathrm{~ns}$, one with the potassium ion restrained in the center of the channel and one with the ion outside the channel.

\subsection{Results and discussion}

\subsubsection{Polyalanine channels}

The PMFs for potassium ion permeation through the series of polyalanine channels are displayed in Fig. 6.4. The average barrier for ion permeation is around $40 \mathrm{~kJ} / \mathrm{mol}$, which is approximately the same free energy barrier reported for gramicidin A embedded in Dimyristoylphosphatidylcholine (DMPC) in the absence of finite size, periodicity and membrane polarizability corrections [182, 192]. It is known that the lack of electronic polarizability in force fields for lipid membranes, and for octane molecules, leads to an overestimation of the free energy barrier for ion permeation $[192,193]$. Because we are primarily interested in the relative free energy barriers for ion permeation, and membrane polarization effects are not coupled to ion-pore interactions [194], no correction was applied.

The profiles display distinct entrance barriers due to the capping groups and the loss of ion solvation. A local minimum, with a binding energy of $\sim 3 \mathrm{~kJ} / \mathrm{mol}$, is located on top of these barriers due to the stabilizing effect of capping groups and the peptide carbonyl charge distribution. Several other transient binding sites are found along the profiles, resulting from the combinations of ion-peptide and water-peptide interactions. 


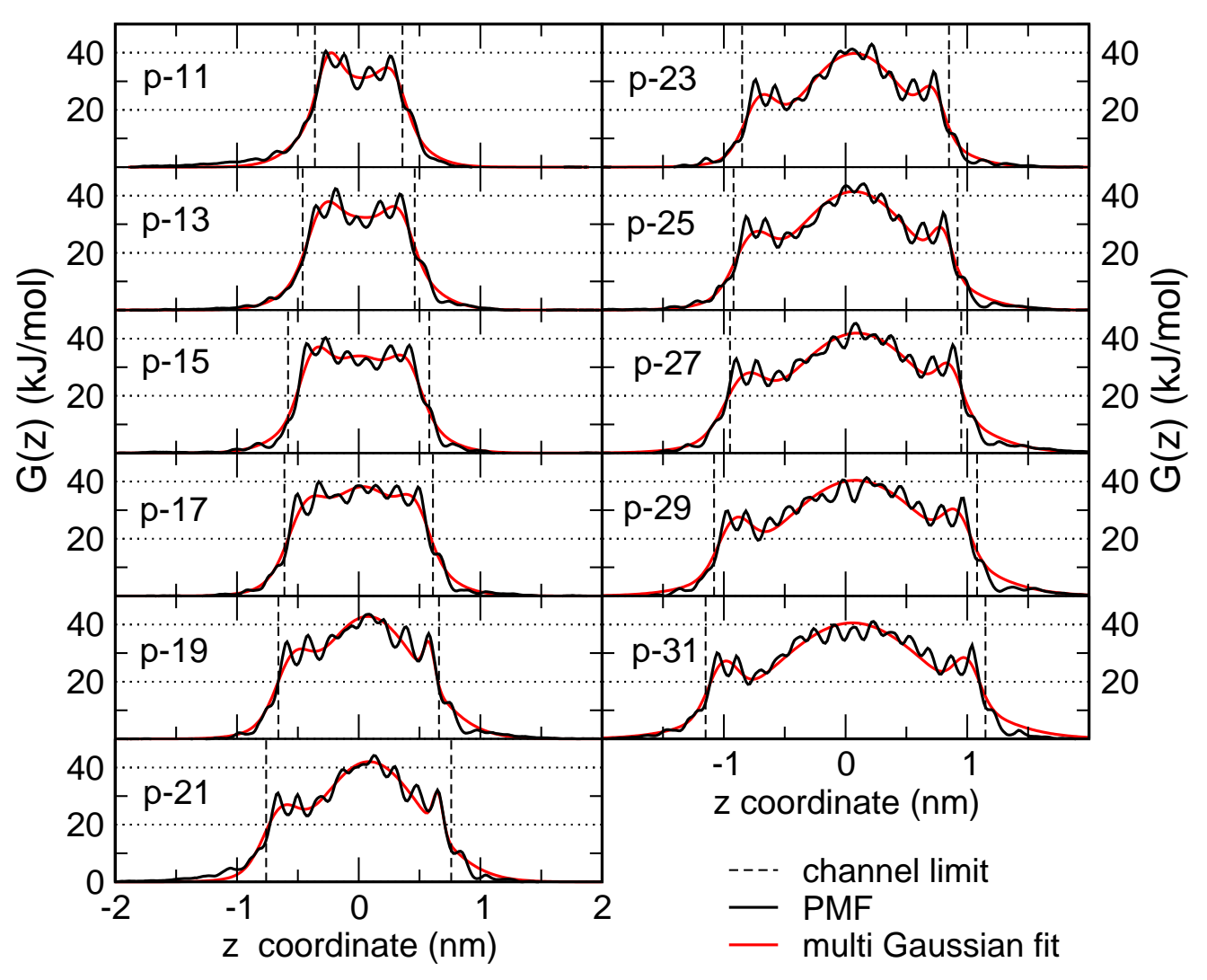

Figure 6.4.: Potentials of mean force (PMF) for potassium ion permeation at $300 \mathrm{~K}$ for the series of polyalanine peptides (black). Underlying each PMF is a 3 Gaussian fit to the main features of the channel (red) : the two entrance barriers (constant) and the central barrier that varies with channel length. Dashed black lines indicate the channel entrance and exit. Errors bars (not shown for clarity) are below $\pm 1.5 \mathrm{~kJ} / \mathrm{mol}$.

The overall free energy barrier increases only slightly with increasing channel length, with $5 \mathrm{~kJ} / \mathrm{mol}$ as the largest difference among the barriers. Further inspection reveals that as the peptidic channel elongates, a central barrier emerges while the entrance barriers decrease, as shown in Fig. 6.5a. Considering a central barrier that increases with the length of the channel, the entrance barriers appear to be added on top of this more general feature. Decomposition of the different PMFs (see Theory and Methods) clearly shows the rise of the central barrier until saturation (red curves in Fig. 6.4, black curve in Fig. 6.5). Such a saturation is predicted by continuum theories [195-198] and it corresponds to the self-image potential (free energy in our case) at infinitely long pores.

For ions in the central position, elongating the channel implies a decrease of the long-ranged electrostatic stabilization of the ion provided by the bulk water. 


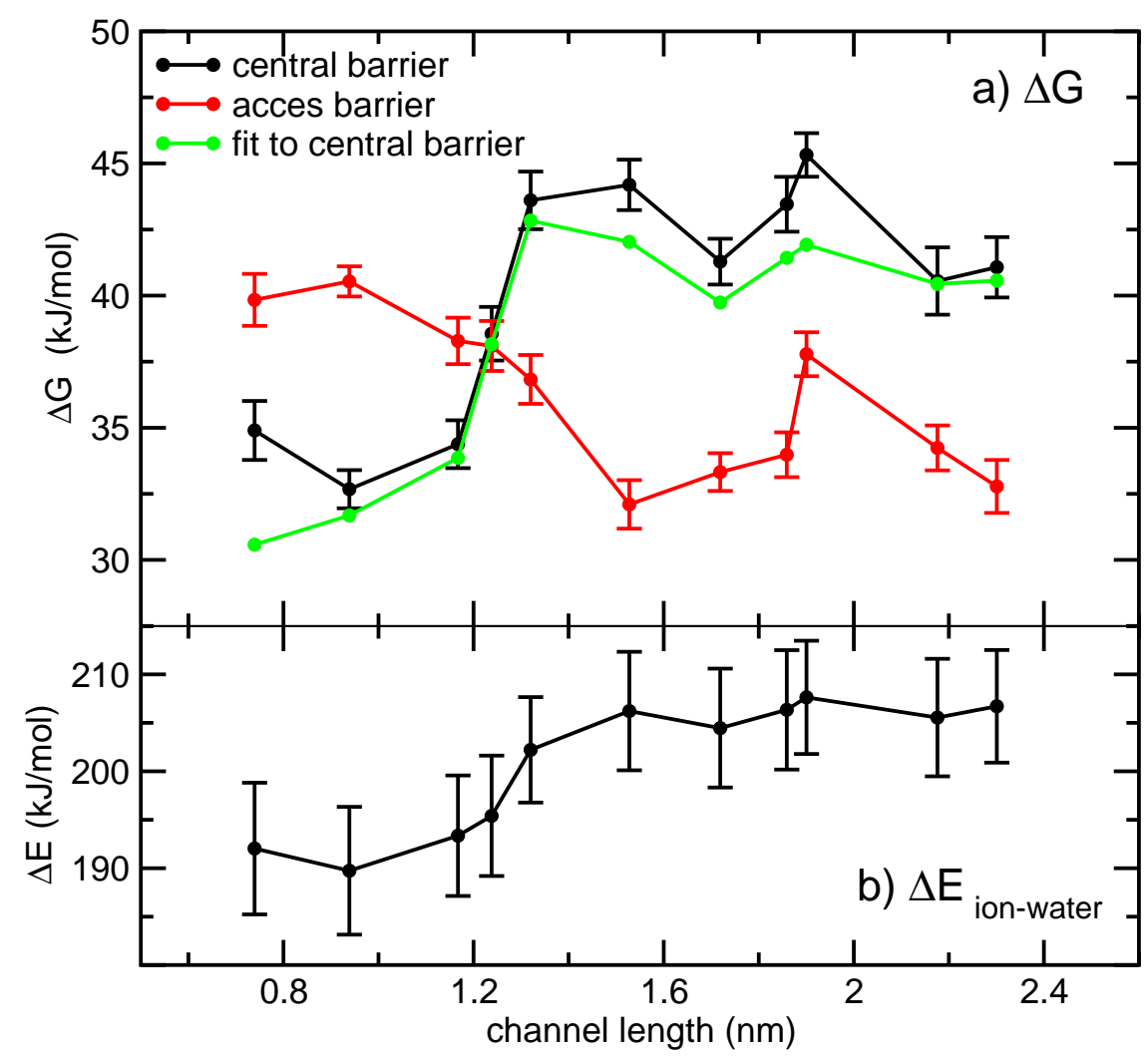

Figure 6.5.: Free energy barriers (a) for ion permeation in polyalanine channels as a function of channel length. The black line in the upper panel indicates the central barrier $\Delta G$, which is dominant in channels longer than p-17. The height of the central Gaussian function fit (see Theory and Methods for details) is displayed in green. Red lines represent the free energy for the access barrier. The difference in ion-water interaction between the ion in the bulk and the ion inside the center of the channel $(b)$ as a function of channel length correlates with the free energy barrier.

Eventually, the presence of bulk water becomes unnoticeable for the ion, and the increasingly destabilizing desolvation effect levels off. The difference between the ion-water potential energy for the ion in bulk and the ion in the channel saturates after p-19, see Fig. 6.5b, correlating with the saturation of the free energy barrier. As previously noticed [199], ion-channel interactions together with the stabilization of the ion by the single-file water column are not able to fully compensate for the loss of the ion's solvation shell.

Construction of the enthalpy barrier for ion permeation from contributions of the different constituents (water-water, water-ion, ion-pore ...) is in principle possible, but challenging due to the large compensating energy terms involved. Instead, we extracted the complete enthalpy contribution to the free 
energy (Fig. 6.6a, red curve) using four representative channels (p-15, p-19, p-25 and p-29) as described in the methods section.
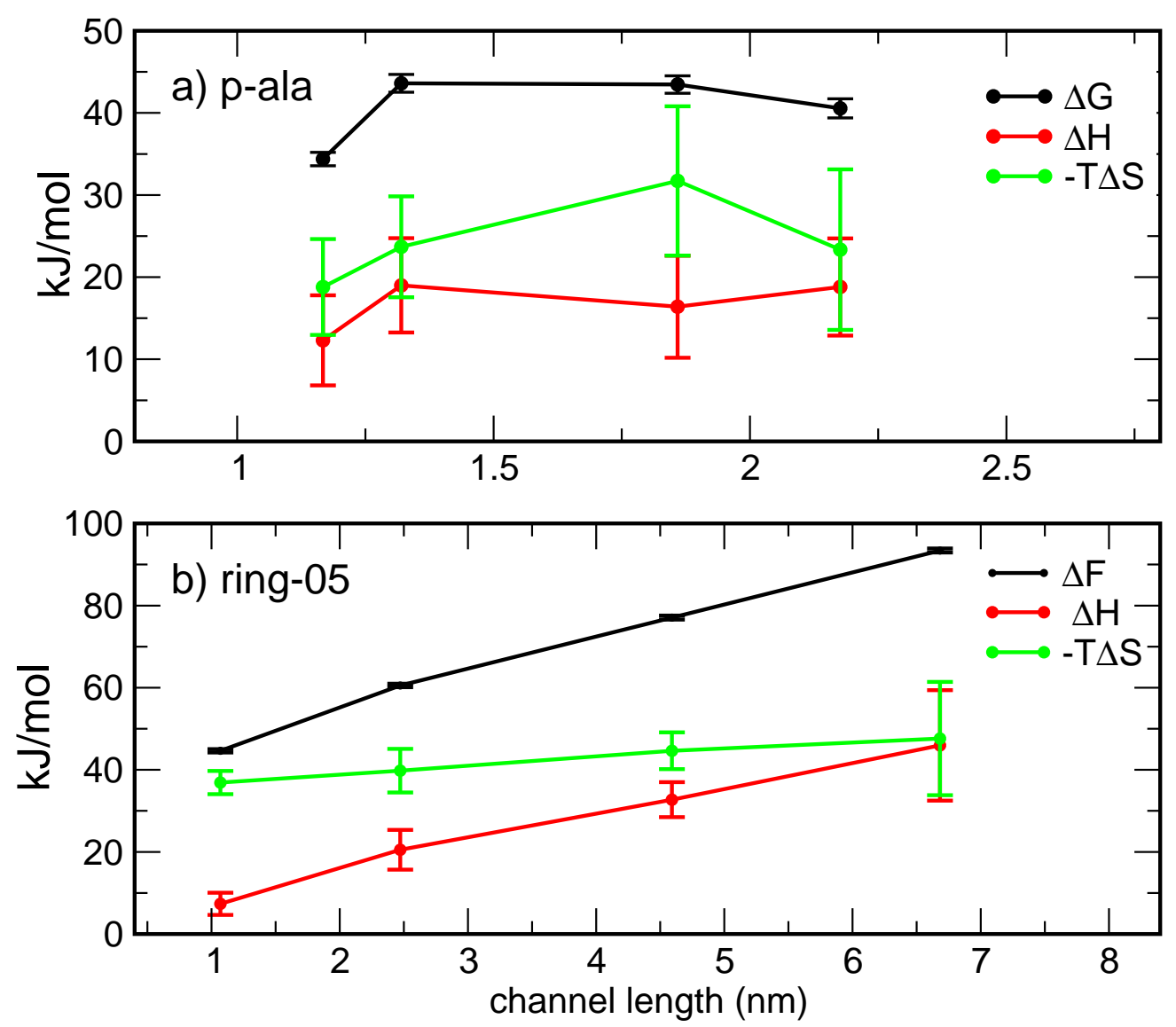

Figure 6.6.: Decomposition of free energy barriers for potassium ion permeation through polyalanine (a) and ring-05 (b) selected channels. The entropy components are weighted with the temperature $(300 \mathrm{~K})$ and sign reversed $(-T \Delta S)$. For the polyalanine channels all thermodynamic quantities could be directly computed from the simulation. For the ring structured channel with charges $\pm 0.5 \mathrm{e}$, only the free energies and the entropies could be extracted independently from the umbrella simulations. The enthalpy contribution of the ring system was computed from $\Delta H=\Delta G+T \Delta S$.

The averaged values for $\Delta H$ show the same saturation trend as the free energy, although subject to large fluctuations, responsible for $\sim 5 \mathrm{~kJ} / \mathrm{mol}$ uncertainty. Entropy estimates, based on temperature dependence of the PMF, reflect a similar tendency, but also suffer from statistical inaccuracy (Fig 6.6a, green curve). Nevertheless, the sum of the two individual components $\Delta H$ and $-T \Delta S$ agrees well with the free energy extracted from umbrella sampling, justifying the use of equation 6.1. Remarkably, there is a reduction of the system entropy when 


\section{Entropy reduction increases single-file ion permeation barriers}

the ion is moved from the bulk to the channel center. Furthermore, the entropy contributes more to the total free energy barrier than the enthalpy. Such a large contribution to the free energy was speculated by Jordan [200, 201], who assigned the effect to the low probability of concerted motions in single-file channels. The reduction of the entropy of the system is directly connected to an increase of the free energy barrier with the temperature, via equation 6.1. Note that this does not imply a decrease of the ion flux with temperature, i.e. an inverse Arrhenius plot. Although the activation free energy required increases with temperature, the ratio $\Delta G(T) / T$ decreases. Furthermore, the diffusive contribution to the ion permeation will also rise with the temperature, yielding normal Arrhenius behavior [202].

\subsubsection{Ring structured channels}

To isolate the influence of the capping groups and hence to focus on the effect of ion desolvation, we investigated simplified channels (see Fig. 6.1b). Figure 6.7a shows the sequence of PMF profiles for artificial channels of increasing numbers of rings (r-02 to r-20). Here we denote the free energies as $F$, since the use of constant volume simulations results in Helmholtz free energies. After a sharp rise of the PMF at the channel entrance, there is a systematic concave increase of the free energy until the ion reaches the central position. Local minima inside the channel correspond to the position of the rings, whereas local maxima are found between ring planes.

Figure 6.8a (black curve) displays the overall free energy barrier, which, surprisingly, increases approximately linearly with the channel length. This is remarkable, since r-20 is $6.6 \mathrm{~nm}$ long, roughly 3 times longer than p-ala29, where a saturation was observed. This behavior is particularly remarkable, since the desolvation penalty for ion permeation, as monitored via ion-water interaction (Fig. 6.8b, black curve), does saturate at approximately r-10. The total enthalpic contribution to the free energy (Fig. 6.6b), which includes the resulting cancellations of large interaction components, shows an almost linear increase, parallel to the free energy vs the channel length. As for the polyalanine channels describe above, we used four representative channels (r-04, r-08, r-14 and r-20) to extract the enthalpy and the entropy terms that account for the free energy barriers. Like the polyalanine channels, the entropy reduction dominates the free energy 


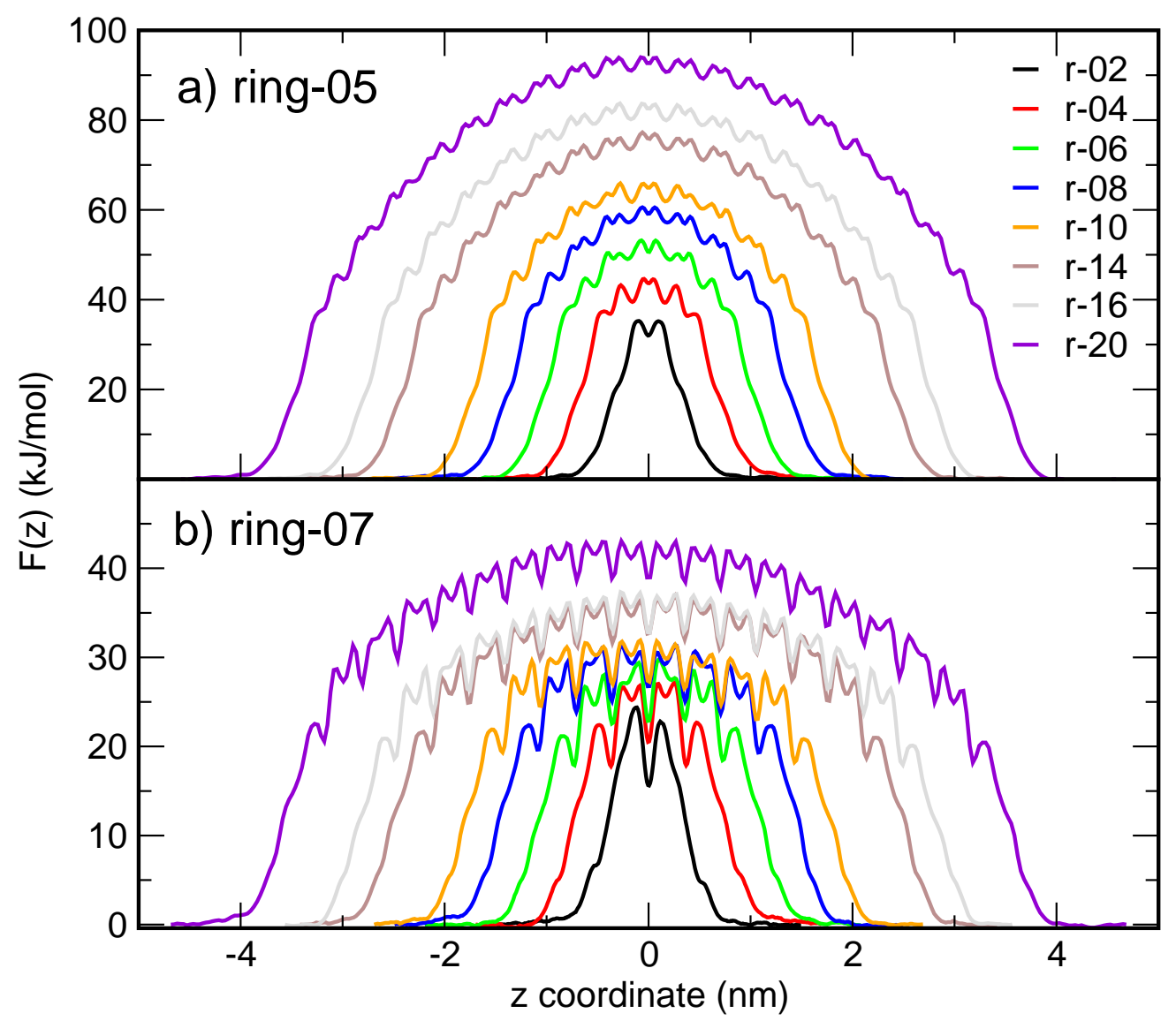

Figure 6.7.: PMFs, $F(z)$, for potassium ion permeation through ring-structured channels. (a) PMFs for ring channels with charges of $+0.5 \mathrm{e}$ on the carbon and $-0.5 \mathrm{e}$ on the oxygen. (b) PMFs for ring channels with charges of $+0.7 \mathrm{e}$ on the carbon, $-0.7 \mathrm{e}$ on the oxygen and increased flexibility along the $z$ coordinate.

barrier. This effect is maximal for the shorter channels, but does not increase as fast as the enthalpic penalty with increasing channel length.

Free energy barriers were found to be higher in the ring-05 channels than for the polyalanine channels, presumably due to the relatively low polarity. A second set of ring channels (ring-07, see Theory and Methods) with augmented charges and larger mobility along the $z$ axis was studied to test this hypothesis. These systems showed much lower barriers (Fig. 6.8a, red curve). Extraction of the ionwater potential energy reveals very similar values to the ones obtained for the ring-05 channels (Fig. 6.8b, red curve). Therefore, the higher interaction energy between ion-channel and water-channel is likely responsible for the reduction of free energy barriers. 
6. Entropy reduction increases single-file ion permeation barriers

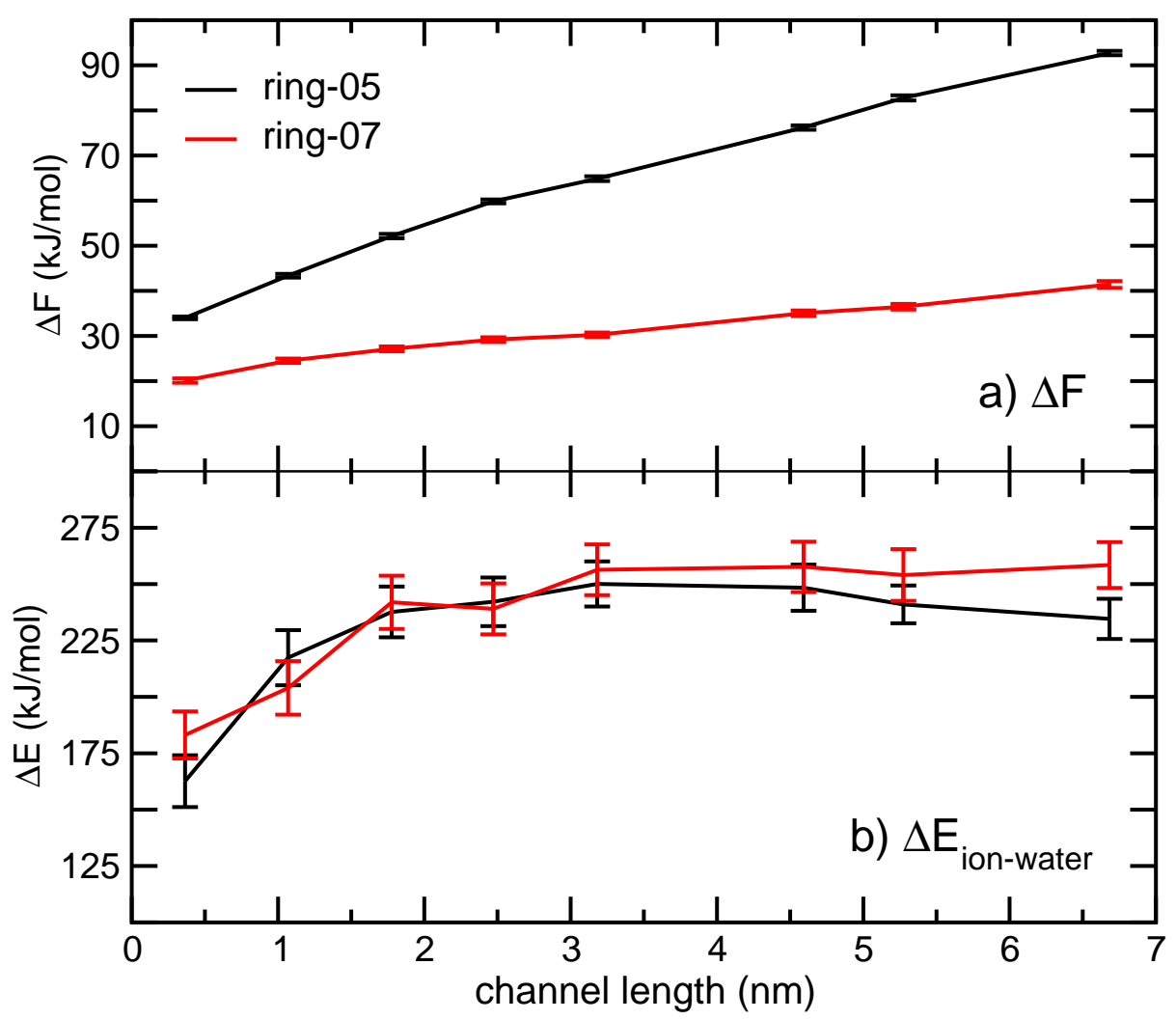

Figure 6.8.: Free energy barriers, $\Delta F$, (a) and ion-water potential energy difference between the bulk and the channel (b) for the ring-structured channels, as a function of channel length.

From the thermodynamic analysis performed on the two types of channels, polyalanine and ring systems, we conclude that the length dependence of the free energy is enthalpy dominated, but the major contribution to the free energy is entropic.

\subsubsection{Origin of system's entropy reduction}

Now that we have established the role of entropy, it is interesting to see which parts of the system contribute most to this entropic cost. There are two components that lead to a significant increase of the system entropy when moving an ion from the bulk to the center of the channel, illustrated in Fig. 6.9 a) and b): (a) removing a potassium ion from the bulk, $17 \mathrm{~kJ} / \mathrm{mol}$ for potassium ion at $300 \mathrm{~K}$ [203], and (b) moving a water molecule from the channel to the bulk to allocate the ion in the channel. Since the overall system entropy decreases, these 
gain in entropy must be surpassed by other entropy reducing effects.

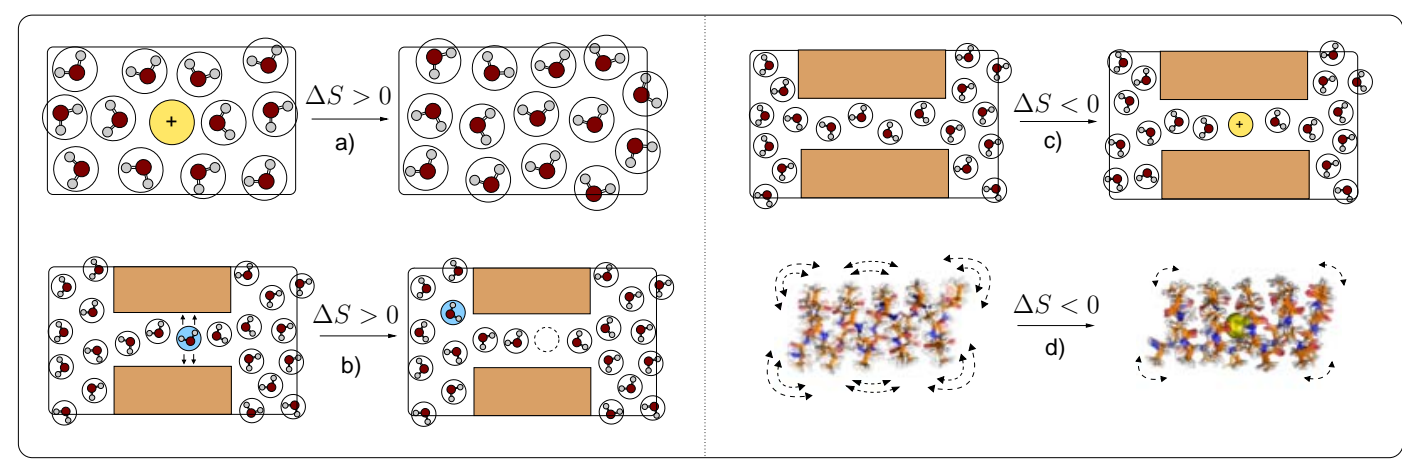

Figure 6.9.: Sketch of possible sources of entropy change. a) and b) correspond to a gain of system entropy caused by removing an ion from the bulk (its presence highly orients the water molecules in the solvation shell) and freeing a water molecule from the channel, respectively. The loss of system entropy could be due to increased polarization of the water molecules inside the channel in the presence of an ion (depticted in panel c), and caused by the reduction of the motions of the channel upon ion binding (illustrated in panel d).

The use of the cylindrical constraint $(0.5 \mathrm{~nm})$ on ion mobility introduces an entropic penalty of $k_{B} T \ln \frac{A_{\text {in }}}{A_{\text {out }}}$, where $A$ is the area sampled by the ion. Since the radius of our channels is around $0.25 \mathrm{~nm}$, the free energy penalty due to narrowing of available sampling area is only $\sim 1.7 \mathrm{~kJ} / \mathrm{mol}$, too small to account for the observed entropy reduction. In the following we discuss the reduction of rotational entropy for water (Fig. 6.9 c)) in the channel and restrictions of channel mobility upon ion binding (Fig. $6.9 \mathrm{~d}$ )) as the major components.

Water dipole moment and rotational water entropy Water molecules are sensitive probes for the electric field generated by an ion and its surrounding media. On average, and in absence of other external influences, water dipoles orient in radially opposed direction from the ion. The degree of orientation depends on the distance from the ion and the permittivity of the medium. Upon a permeation event of an ion through the channel, the rotational freedom of the water molecules inside the channel is reduced. The effect is maximized when the ion is in the central position. The reduction of water rotational freedom in single-file channels in presence of ions has been long recognized [204], as well as the need to incorporate explicit water dipoles in semi-microscopic modeling for ion permeation [205]. 


\section{Entropy reduction increases single-file ion permeation barriers}

From 4 independent $8 \mathrm{~ns}$ MD simulations for each channel, we extracted timeaveraged position dependent dipole moments of water inside the pore. During these simulations, the ion was restrained at the center of the channel. Figure 6.10 displays the averaged projection of water dipoles onto the pore axis $\left(\left\langle\mu_{z}\right\rangle\right)$ for all studied channels as function of their position in the pore. The water dipole was normalized with respect to the total dipole of the TIP4P water model $(2.177 \mathrm{D})$. In the vicinity of the ion (located at 0 in the $x$ axis), all neighboring water molecule dipoles point away from the ion.

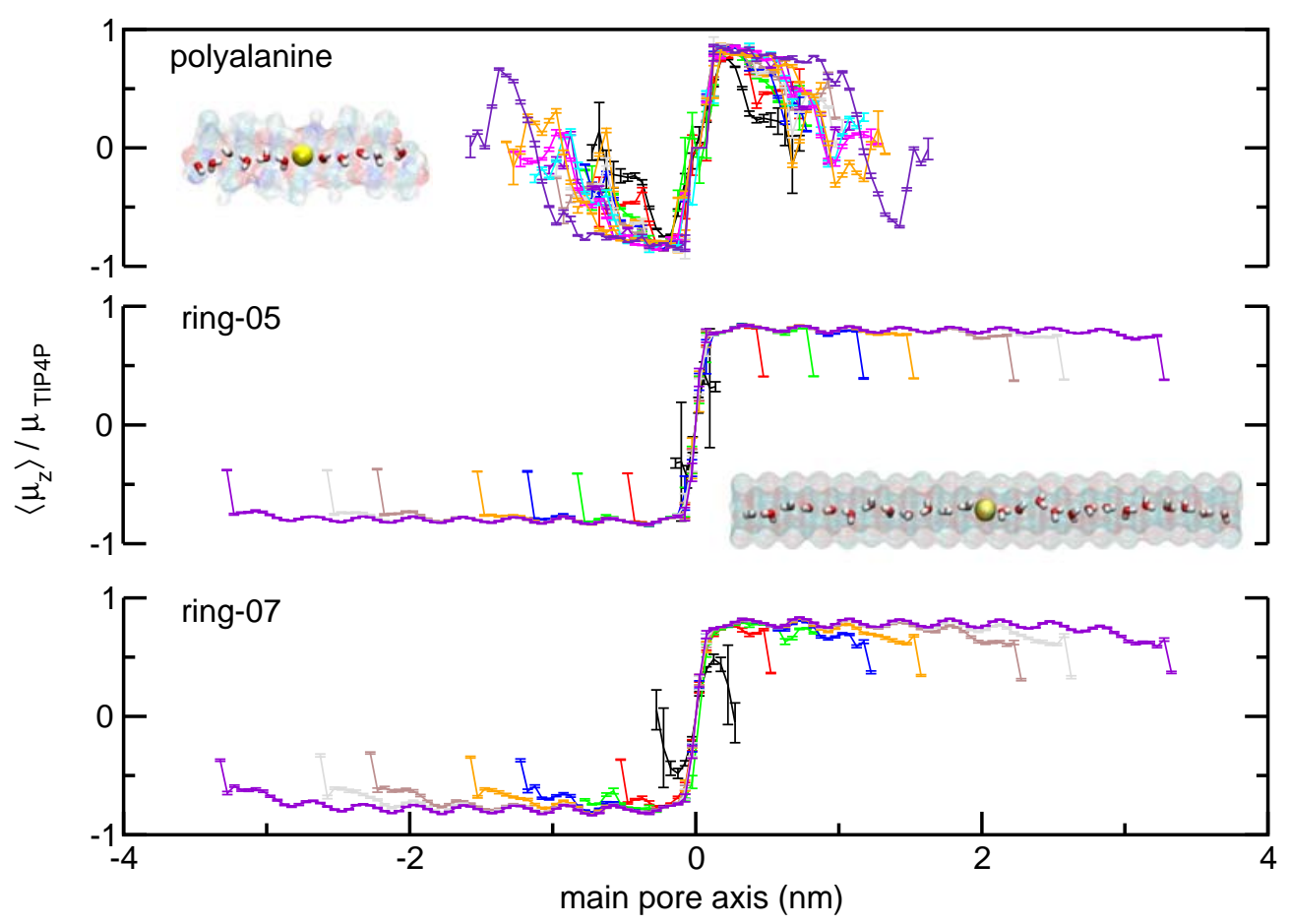

Figure 6.10.: Projection of the average dipole moment, $\left\langle\mu_{z}\right\rangle$, of water molecules inside the channel onto the pore axis for the polyalanine channels (upper panel), the ring-05 (center) and the ring-07 (lower panel) channels. The water dipole was normalized with respect to the total dipole of the TIP4P water model $(2.177 D)$. Different colors indicate different channels of increasing length. Profiles have been symmetrized. On the upper left, a typical simulation snapshot shows the water orientation when the ion (yellow sphere) occupies the central position. As seen from the $\left\langle\mu_{z}\right\rangle$ curves, water molecules far from the ion loose their alignment with the pore axis in the polyalanine channels due to a decay of the electric field and interactions with the bulk water (not shown). The central and lower panels illustrate the long ranging polarization induced by the potassium ion for the ring channels. 
There are significant differences in the behavior of the electric field generated by the ion in the peptidic and ring-structured channels. For the polyalanine pores, the influence of the electric field drops quickly after the first two water molecules around the ion. Water molecules lying at the interface region with the bulk are no longer polarized by the ion, and adopt orientations induced by the bulk water. Similarly, the ring channels exhibit strong ordering of water dipoles around the ion. In contrast to the peptidic channels, the long-ranged ordering of water molecules reveals that the electric field propagates further through the single-file. Increasing the polarity and mobility of the channel reduces the extend of water dipole ordering, since ring-07 $\left\langle\mu_{z}\right\rangle$ of water at the bulk interface drops by $\sim 0.25 \mathrm{D}$ on average with respect to the ring-05 systems.

Due to the imposed ordering of water molecules caused by the ion, the rotational entropy of water molecules in the channel is reduced. We used the first order uncorrelated rotational probability density (see 6.2 and Appendix A), as a crude estimate for the entropy reduction of water molecules inside the channel. Since dipoles are in fact correlated, this first order uncorrelated probability density is an upper bound for the entropy reduction inside the channel. The results show a decrease of entropy that yields an energetic cost $(-T \Delta S)$ of $\sim 2.3 \mathrm{~kJ} / \mathrm{mol}$ (at $300 \mathrm{~K}$ ) for each water molecule in the polyalanine channels. In the ring-05 system, the entropy reduction per water molecule is higher in the shorter channels, $\sim 3.8 \mathrm{~kJ} / \mathrm{mol}$ at $300 \mathrm{~K}$, and drops to $\sim 2.2 \mathrm{~kJ} / \mathrm{mol}$ for the longest channel. Considering the water occupancy for each channel, the overall contribution to the entropy for polyalanine channels ranges from 10 (p-15) to $18 \mathrm{~kJ} / \mathrm{mol}(\mathrm{p}-29)$, and from 15 (r-04) to $55 \mathrm{~kJ} / \mathrm{mol}(\mathrm{r}-20)$ for the ring-05 system. These values, although only qualitative, suggest a substantial contribution to the reduction of the system entropy.

Reduction of channel mobility Although we made use of position restraints to stabilize the pore structure of our models, the electrostatic interactions between the channel and the permeating ion have a significant effect on the mobility of the pore. Mass-weighted Principal Component Analysis reveals substantially reduced amplitudes in the channel atomic fluctuations in the presence of a potassium ion. For the polyalanine channels, the entropy loss at $300 \mathrm{~K}$ ranges from 10 (p-15) to $28 \mathrm{~kJ} / \mathrm{mol}$ (p-29), and the ring-05 channels show an entropy decrease 
between $14(\mathrm{r}-02)$ and $25 \mathrm{~kJ} / \mathrm{mol}(\mathrm{r}-20)$. To a qualitative extend, these results provide a strong indication that channel mobility is reduced in the presence of the ion in its lumen. Entropies computed using the Schlitter formula and and the $\mathrm{QH}$ approximation agree within $0.2 \mathrm{~kJ} / \mathrm{mol}$.

To conclude, the entropic contributions presented are an upper bound, but they provide a strong indication that channel mobility and rotational water freedom in the channel are reduced in the presence of the ion in its lumen, which leads to an overall system entropy reduction upon ion permeation.

\section{Implications for biological channels and for the design of new ion channels}

Finally, it is of interest to compare the presented results with experimental findings and to discuss the implications for biological channels and the design of novel channels. The selectivity filter of naturally occurring channels, such as the bacterial potassium channel KcsA, is shorter (1.2 nm in length) than the lipid bilayer in which it is embedded [170]. Apart from ion stabilizing helical dipoles and multiple ion occupancies [62, 170, 206-208], a short selectivity filter contributes to the high ion rate [27].

Qualitative agreement with experimental ion flux through single-file channels of different length can be found in ion conductance through gramicidin A derivatives minigramicidin and midigramicidin [64]. In $1 \mathrm{mM} \mathrm{KCl}$ solution, embedded in a mixture of DMPC and E. coli lipid extract, minigramicidin, $\sim 1.8 \mathrm{~nm}$ in length, has a conductance of $0.27 \mathrm{pS}$, and midigramicidin, $\sim 2 \mathrm{~nm}$ long, shows a conductance of $0.20 \mathrm{pS}$.

In order to design ion channels for technological applications, the efficiency of ion transport through the channel can be modulated by adapting the single-file length, the polarity and the flexibility of the pore. Short, polar and flexible channels will permeate ions at a higher rate than long, stiff, apolar channels. Furthermore, since single-file water mobility under osmotic conditions is virtually invariant to the pore length [150, 209], long single-file channels would allow fast flow of water molecules while effectively avoiding the passage of ions, thereby shifting the ion/water selectivity. Such length variability and fixed radius can be experimentally implemented, e.g., in carbon nanotubes. 


\subsection{Summary and conclusions}

The influence of the channel length on potassium ion permeation depends on the nature of the channel. Replacement of the solvation shell in bulk can not be fully compensated by the carbonyl fragments inside peptidic channels and the single-file of water molecules. In peptidic channels, the saturation of the free energy barrier with increasing channel length mainly arises due to a parallel saturation of the reduced ion-water interaction. In contrast, more rigid channels with similar charge distribution fail to stabilize the barrier for ion permeation, leading to a constant increase of the PMF barrier with the channel length.

The decomposition of the free energies into enthalpic and entropic contributions helps to understand the origin of the permeation barriers. In gramicidin-like channels, and also in the ring-structured channels, the entropy barrier is actually higher than the enthalpic barrier. Although an increase in the entropy of the water is gained after removing the ion from the bulk, the combination of channel and water mobility reduction finally lowers the system's total entropy as the ion permeates the channel.

The general picture emerging from our study is twofold: while a reduction of the system entropy as the ion passes the channel is the major contribution to the permeation barrier, the ion permeation length dependence is dominated by the enthalpic component of the free energy. 
6. Entropy reduction increases single-file ion permeation barriers 


\section{Summary and Conclusions}

Water and ion channels are of vital importance for life. They are ubiquitous gates that allow the passage of charged or polar species through the lipidic membrane of cells, which would be otherwise largely impermeable ${ }^{1}$. The evolutionary imperative endowed ion and water channels with selective narrow pathways, where the flux of matter is efficiently filtered. To accomplish this task, the permeation pathway usually restricts the passage of molecules in a single-file fashion, forcing a direct interaction between possible permeants and the channel. Due to their small size and specificity, ion and water channels are also of high interest in technological applications, ranging from selective nanofluidic filters to sensible detectors.

Motivated by the biological and technological relevance of water and ion channels, we performed a systematic characterization of the determinants of water and ion permeation through pores of Molecular Dimensions. The method applied, molecular dynamics with a molecular mechanics force-field, allows a time resolved atomistic description of the permeation process. Furthermore, it generates welldefined correct ensembles for the calculation of thermodynamic properties by means of statistical mechanics. Using rate theory and statistical thermodynamics, relevant properties of the studied systems were characterized, such as the water permeability and free energy barriers.

The most prominent property of water channels, the osmotic permeability, is a measure of the response to a concentration gradient between the compartments connected by the channel. To determine osmotic permeabilities from equilibrium simulations, $i$. e., without imposing a concentration gradient, different methodologies were applied throughout the thesis depending on the nature of the permeation regime. All these methods are concerned with the identification of a collective coordinate that describes the evolution of water molecules along the

\footnotetext{
${ }^{1}$ Depending on the polarity of the permeants and on the composition of the lipidic membrane.
} 


\section{Summary and Conclusions}

pore axis. This approach, as opposed to an explicit computation of the relationship between flux and concentration gradient, has the positive side effect that allows the simultaneous calculation of the diffusive permeability in addition to the osmotic permeability.

Our starting point was the study of gramicidin A in a helical dimer conformation (gA-HD) and the cross-linked midigramicidin (MDg), which is four residues shorther than gA. These peptidic channels permeate water in the singlefile regime, and are sufficiently well-characterized experimentally: their threedimensional structure is known from NMR spectroscopy, providing initial coordinates for our simulations, and their water permeabilities are known from electrophysiological experiments, which allows a direct comparison with the results from our simulations. Both peptides were embedded in a model lipid membrane (DMPC) to mimic the experimental conditions. The peptidic channels were found to be conformationally stable during the simulations, with the linked MDg displaying greater stability than gA-HD. The most flexible part of the pores were identified to be the $\mathrm{N}$ - and C-terminal capping groups, which were found to partially deviate from their original positions, causing the channel to block or reduce its permeability. We found that the modulation of the membrane thickness due to lipid-peptide interactions depends on the length of the channel, MDg causes a local thinning of the membrane around it and a thickening at larger distances, whereas the gA-HD impact on the membrane is smaller and causes a slight overall increase in the bilayer thickness.

The tryptophan residues from the peptidic channels were found to have a dominant role in the anchoring of the peptides in the membrane, due to their amphiphilic character, in agreement with previous computational and experimental studies. The fluidic nature of the lipid bilayer and the interactions between peptide residues and lipid molecules, particularly between the tryptophan residues and the glycerol moiety of the lipid, leads to a tilting of the peptides with respect to the membrane normal. The combination of the peptide tilt and the membrane thinning/thickening results in the lipid head groups transiently blocking the channel entrance and hence affecting their water permeability. This observation can be used to design more efficient water channels with altered membrane anchors, and by extension also ion channels. Suggested modifications of the gramicidin peptide are the substitution of non-tryptophan residues close 
to the pore entrance for lipid-like molecules (e. g. esters with a long alkyl chain), to prevent the surrounding membrane lipids to approach the channel entrance.

The osmotic permeability coefficients computed from our simulations agree qualitatively with the experimental results: MDg permeates at faster rates than gA-HD. Although several reasons that could explain this effect were discussed, such as channel geometry and stability, a clear structure-activity relationship is obscured by the multiple differences between the channels. Apart from the length, also the differences in the capping groups and channel radius might affect the water permeability. These results evidenced the need of a systematic approach to determine the specific role of the pore geometry and the polarity on the permeability properties of narrow pores.

The first property that was systematically studied as possible determinant for the permeability in single-file pores was the pore length. This was motivated by recent experimental measurements of water osmotic permeability coefficients for cross-linked minigramicidin, midigramicidin, and gramicidin A. The experiments reported an exponential length dependence of the water mobility: reduction of pore occupancy by one water molecule drastically enhances the water permeability. This result could not be explained by text book models, where the relationship between osmotic permeability coefficient and the length was predicted to be inversely proportional. To test whether the experimentally observed dependence was solely due to changes in pore length, polyalanine peptides of increasing length and folded as the $\beta$-helix conformation of a monomer gA-HD were designed. To render the systems directly comparable, we used soft position restraints to ensure the stability of the peptide fold. To control the blocking effect of the lipid head groups and to have full control of the peptide-membrane match, an artificial membrane of octane molecules was used.

Our results indicate that the length of the channel alone does not have a direct impact on the water mobility, quantified by the osmotic permeability coefficient. The thermodynamic analysis of the water permeation reveals that the main permeation barrier are located at the channel entrances, rather than concentrated in a series of water binding sites. This access barrier was found to be independent of the channel length, thereby explaining the observed length-independence. This dominant access barrier originates from an incomplete compensation of the loss water-water interactions by the channel when a water molecule moves from the 


\section{Summary and Conclusions}

bulk to the channel. Due to collectivity, the reported single-molecule potentials of mean force do not reflect the underlying effective free energy profile. For this reason, we constructed several scenarios corresponding to different underlying free energy profiles to test their resulting length dependence. We found that different scenarios could explain our simulations results, the experimental results and the text book model. Our study suggests that rather than changes in the channel length, water permeability can be modulated by modifications of the channel entrance, supporting the view that a well defined pore access is crucial for water permeation.

A subset of the polyalanine channels used to study the length dependence were selected to investigate the role of the polarity. To do so, we varied the dipole moment of the carbonyl groups of the peptidic backbone. By changing the affinity between the water molecules and the channel, the average occupancy of water molecules in the channel was found to be modulated. Time-averaged pore occupancies reflect open/closed states of the pore, rather than configurations where holes interrupt the single file configuration. An adsorption curve was used to describe the normalized time-averaged pore occupancy as a function of the dipole moment of the peptidic carbonyl groups. The effect of the polarity on the permeability coefficients was found to be strong: low and high pore polarities resulted in low permeabilities, originated by the reduced pore occupancy and high 'binding' energy, respectively. In spite of the different behavior of the water molecules in the channel for different pore polarities, no significant effect of the channel length was found also for different polarities. The water permeability was reported to be maximal for pore polarities slightly lower than the polarity of naturally occurring peptidic backbones, which corresponded to pore occupancies of $\sim 75 \%$. This result indicates that naturally occurring pores with a water pathway formed by carbonyl groups possess a fine-tuned hydrophilicity for a near-optimal permeation of water molecules.

The geometric characteristic of the pores that was found to be most critical for the water permeability is the pore radius. By exploring a large set of pore radii and polarities for model pores, several permeation regimes became apparent, ranging from almost empty to permanently water filled pores. The water density in the pores was found to oscillate with the radius: the single-file configuration displays a maximum in the density at the radius where the single-file 
configuration is optimally packed. The transition towards a double-file regime is characterized by a minimum in the density as a function of the pore radius. A further increase of the pore radius results in the water pore density converging towards the density in bulk water. Although the overall radial dependence is qualitatively similar for all pore polarities, the polarity of the channel determines at which radius the transition between different permeation regimes takes place.

The osmotic permeability coefficients as a function of the pore radius were found to steadily increase with the pore radius. The diffusive permeability, however, shows a minimum at the pore radius where the single-file configuration is the densest. For pores with a radius below the densest single-file configuration, the channel permeability depends on the polarity in the same fashion as described for the polyalanine channels. At larger pore radii, low-polarity pores exhibit high water permeabilities, and the overall radial dependence converges to the macroscopic radial dependence.

The ratio of osmotic and diffusive permeabilities was found to be proportional to the average water occupancy for all combinations of pore radius, pore length and pore polarity in the single-file regime. This result can be of practical application to experimentally infer the state of the permeating water molecules $(e . g$., completely water filled pores or liquid-vapor oscillations). If the obtained ratio of osmotic and diffusive permeabilities for single-file pores does not agree with the occupancy computed from pure geometrical considerations, it implies that the channels occupancy oscillates with time, indicating liquid-vapor oscillations.

In contrast to the invariance of the single-file water mobility as function of the pore length, ion permeation in such narrow peptidic channels shows a strong length dependence. The increase of the free energy barrier for ion permeation is correlated with the loss of ion-water interaction for peptidic channels. Since increasing the channel length implies larger ion-bulk water distances as the ion permeates the channel, the energetic penalty to the free energy due to a loss of electrostatic stabilization increases with channel length until saturation. A thermodynamic analysis revealed that both the enthalpy and the entropy contribute to the increase in the free energy barrier for ion permeation. The entropy decrease was assigned to reduction in conformational flexibility of the channel and to a decrease of the rotational water entropy upon ion permeation. Although the entropic contribution is the largest component of the free energy barrier, the 
length dependence is dictated largely by the enthalpy.

The acquired knowledge on the permeability determinants can be readily used for the design of efficient water channels. If the channel is not required to be selective, apolar channels with large radius are the most efficient in permeating water molecules. If a single-file is required, channels of relatively low polarity preserve the single-file at larger radius, allowing faster permeation rates. We found that the length of the channel does not play a significant role in determining the water permeability. If the channels are embedded in a lipid membrane, this implies that they can be made sufficiently long to match the membrane thickness, thus avoiding interruptions on the water permeability due to lipid head groups. Furthermore, the water permeability can be tuned by modifying the polarity of the channel, e. g., by including polar/apolar fragments along the water pathway. The fact that water permeation is largely length-independent and that ion permeation is strongly length dependent, implies that ion/water selectivity can be achieved through length modulation. 


\section{A. Approximate calculation of the rotational water entropy}

In order to quantify the reduction of the rotational freedom of a water molecule due to the presence of an ion, several approximations need to be carried out to render the problem treatable. A rigorous calculation of the contribution of a particular degree of freedom to the overall system entropy is not a trivial task [69]. Here, we approximate the true value of the entropy by assuming that several contributions are uncorrelated. The absolute entropy of a system, $S=-k_{B} \int_{\Omega} d \vec{x} d \vec{p} p(\vec{x}, \vec{p}) \ln p(\vec{x}, \vec{p})$, is determined by its phase space density $p(\vec{x}, \vec{p})$, where $\vec{x}$ and $\vec{p}$ represent the positions and momenta of all the particles, with $k_{B}$ being the Boltzamn constant, and the integration is done over the phase space $\Omega$. We are here interested in the contribution of only a few degrees of freedom, and therefore we factorize the total density distribution into individual contributions, the density corresponding to the rotational degrees of freedom of all water molecules in the channel, those in the rest of the system and a coupling or correlation term: $p_{\text {sys }}=p_{\text {rot }} p_{\text {rest }} p_{\text {corr }}$. As a first approximation, we do not consider the correlation between the subsystems, $p_{\text {corr }}=1$. With the assumption that the factorization is valid, the entropy of the system can be separated into $S_{\text {sys }}=S_{\text {rot }}+S_{\text {rest }}$. In order to evaluate the rotational density of $N$ water molecules, we need to obtain an estimate of the probability density for all rotational states of all water molecules, which we denote as $p_{\text {rot }}\left(\omega_{1}, \omega_{2}, \ldots, \omega_{N}\right)$, where $\omega_{N}$ refers to all rotational degrees of freedom for the water molecule $N$. An exact quantification of this probability density is complex due to the multiple couplings between all degrees of freedom. To a very first approximation, we factorize the rotational probability into products of equal and independent probabilities, $p_{\text {rot }}\left(\omega_{1}, \omega_{2}, \ldots, \omega_{N}\right) \approx \prod_{i}^{N} p_{\text {rot }}\left(\omega_{i}\right) \approx N p_{\text {rot }}(\omega)$. The overall rotational entropy for a channel containing $N$ water molecules is then $N S_{\text {rot }}$. 


\section{A. Appendix}

Because we are requiring the water molecule to be in some place inside the channel, the probability distribution actually depends on the position of the water molecule under observation. We use the channel axis as the reaction coordinate, therefore the position is written as a function of $z$, and the density is $p_{\text {rot }}(\omega, z)$. This density, or probability, can be computed as $p(\omega, z)=p(z) p(\omega \mid z)$. The first term, $p(z)$, is the probability to find a water molecule in that particular position $z$, and $p(\omega \mid z)$ is the conditional rotational probability density of one water molecule to be at position $z$ having rotational degrees of freedom $\omega$.

The probability to find a water molecule at position $z, p(z)$, is determined from a molecular dynamics trajectory as a normalized histogram along the reaction coordinate. Since the reaction coordinate is linear, the binning of the positions of the water molecules into $q$ regions of equal (or known) length is straightforward: $p(z)=m(z) / l$, where $m(z)$ is the number of water molecules that fall in the bin at position $z$, and $l$ is the total number of water molecules at any position $z$. Here we assumed bins of the same length.

To define the rotational state of a water molecule inside the pore, we use the dipole moment of the water molecule, defined in cylindrical coordinates by the angles $\phi$ and $\psi$. To take all three degrees of freedom into account, we need to consider the rotation around the dipole, described by the angle $\theta$. We consequently express our conditional probability as $p(\phi, \psi, \theta)=p(\phi, \psi) p(\theta \mid \phi, \psi)$. The inclusion of the rotation around the dipole moment of a water molecule requires a much larger number of events to achieve convergence. Since the first order effect leading to a decrease in rotational entropy is due to the alignment of the dipole with the electric field generated by the ion, we neglected this contribution in our final results. Therefore, the rotational probability we need to evaluate is $p(\mathbf{x})$, were $\mathbf{x}$ is the cartesian coordinate of the dipole moment on a unit sphere.

The last bottleneck to compute our approximated entropies is to obtain a reliable estimate of the probability density $p(\omega \mid z)$, evaluated as $p(\mathbf{x} \mid z)$. In order to compute a probability distribution on a sphere by a histogram method, we would need to divide the sphere surface in partitions of known area. To obtain the best description of the data, an optimal bin area needs to be determined, based for example in minimizing the mean squared error [210]. Since the dipole moments of the water molecules can be highly oriented, and given the limited number of data points, there will be a large number of empty bins, which would 
be problematic in order to extract the entropy from the probability distribution. An alternative approach uses nonparametric density estimates based on a series of probe positions, such as the $k$-nearest neighbor method.

We laid an homogeneously distributed set of probe positions on a sphere, starting from a random distribution of $k$ points placed at positions $\mathbf{x}$. The set of points was treated as particles on a sphere, and using a steepest-descent algorithm they were distributed maximizing all distances between them. We implemented the nonparametric density estimate on the sphere proposed by Ĕgecioğlu and Srinvasa [211], that uses the cosine of the angle $\alpha_{\mathbf{x}} \mathbf{X}_{i}$ between a data point $\mathbf{X}_{i}$, the center of the sphere and a reference bin or probe $\mathbf{x}$,

$$
p_{n}(\mathbf{x} \mid z)=\frac{1}{n A_{m}} \sum_{i=1}^{n} \cos ^{2 m}\left(\frac{\alpha_{\mathbf{x}} \mathbf{X}_{i}}{2}\right)
$$

where $A_{m}=4 \pi /(m+1)$ is the normalization factor, $n$ is the number of data points, and $m$ is a smoothing factor related to the number of probe points. The optimal value for $m$ is $\sqrt{n}$, determined by requesting that the integrated mean square error tends to zero at infinite number of data points.

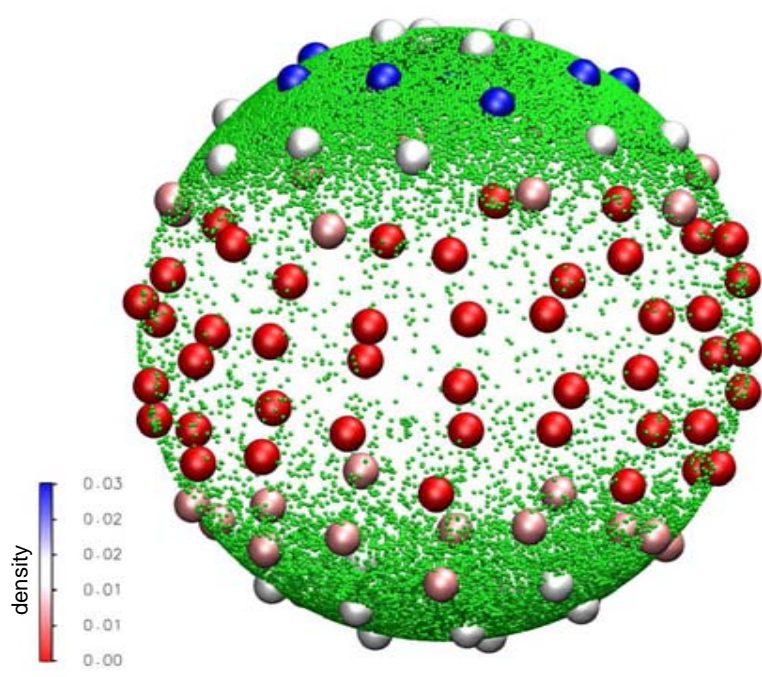

Figure A.1: Illustration of the distribution of water dipole orientation on a sphere (green balls) and the corresponding density computed at homogeneously distributed bins (colored balls). The water molecules are clearly oriented along two distinct directions, which is captured by the density estimate.

From the normalized rotational probability distribution, and the positional probability distributions, the approximation of the rotational entropy contribu- 


\section{A. Appendix}

tion of a water molecule in the channel is

$$
\begin{aligned}
& p_{i}(\mathbf{x}, z)=\sum_{j}^{k} p_{i}(z) p_{n}\left(\mathbf{x}_{j} \mid z\right) \\
& S_{\text {rot }}=-k_{B} \sum_{i}^{q} p_{i}(\mathbf{x}, z) \ln p_{i}(\mathbf{x}, z)
\end{aligned}
$$

The final step is the construction of a reference state for the entropy estimate. This can be defined by an homogeneous distribution of positional and rotational states of the same number of partitions to compute the density. Since we are interested in the change of rotational entropy as the ion permeates the channel, the reference state was defined by the rotational entropy of the water molecules in absence of the ion. 


\section{Bibliography}

[1] A. V. Krylov, P. Pohl, M. L. Zeidel, and W. G. Hill. Water permeability of asymmetric planar lipid bilayers: leaflets of different composition offer independent and additive resistances to permeation. J. Gen. Physiol., 118(4):333-340, Oct 2001 .

[2] W. Pfeffer. Osmotische Untersuchungen. Engelmann, Leipzig, 1877.

[3] J.W. van't Hoff. Die rolle des osmotischen druckes in der analogie zwischen lösungen und gasen. Z. physik. Chemie, 1:481-493, 1887.

[4] E. Overton. Über die osmotischen eigenschaften der zelle in ihrer bedeutung für die toxikologie und pharmacologie. Z. Phys. Chem., 22:189-209, 1896.

[5] A. Finkelstein. Water movement through lipid bilayers, pores, and plasma membranes. Wiley \& Sons., New York, 1987.

[6] W. D. Stein and J. F. Danielli. Structure and function in red cell permeability. Discuss. Faraday Soc., 21:238-251, 1956.

[7] B. Hille. Ion channels of excitable membranes. Sinauer: Sunderland, MA, 3rd edition, 2001.

[8] B. Hille, C. M. Armstrong, and R. MacKinnon. Ion channels: from idea to reality. Nat. Med., 5(10):1105-1109, Oct 1999.

[9] R. MacKinnon. Potassium channels and the atomic basis of selective ion conduction (nobel lecture). Angew. Chem. Int. Ed., 43(33):4265-4277, Aug 2004.

[10] P. Agre. Aquaporin water channels (Nobel Lecture). Angew. Chem. Int. Ed., 43(33):4278-4290, Aug 2004.

[11] G. M. Preston, T. P. Carroll, W. B. Guggino, and P. Agre. Appearance of water channels in Xenopus oocytes expressing red-cell CHIP28 protein. Science, 256:385-387, 1992. 
[12] C. D. Pivetti, M. R. Yen, S. Miller, W. Busch, Y. H. Tseng, I. R. R Booth, and M. H. Saier. Two families of mechanosensitive channel proteins. Microbiol. Mol. Biol. Rev., 67(1):66-85, Mar 2003.

[13] I. R. Booth, M. D. Edwards, S. Black, U. Schumann, and S. Miller. Mechanosensitive channels in bacteria: signs of closure? Nat. Rev. Microbiol., 5(6):431-440, Jun 2007.

[14] M. J. Caterina, M. A. Schumacher, M. Tominaga, T. A. Rosen, J. D. Levine, and D. Julius. The capsaicin receptor: a heat-activated ion channel in the pain pathway. Nature, 389(6653):816-824, Oct 1997.

[15] R. Latorre, S. Brauchi, G. Orta, C. Zaelzer, and G. Vargas. ThermoTRP channels as modular proteins with allosteric gating. Cell Calcium, 42(4-5):427-438, 2007.

[16] E. Lingueglia. Acid-sensing ion channels in sensory perception. J. Biol. Chem., 282(24):17325-17329, Jun 2007.

[17] O. Beckstein, P. C. Philip C Biggin, P. Bond, J. N. Bright, C. Domene, A. Grottesi, J. Holyoake, and M. S. P. Sansom. Ion channel gating: insights via molecular simulations. FEBS Lett, 555(1):85-90, 2003.

[18] O. Beckstein and M. S. Sansom. A hydrophobic gate in an ion channel: the closed state of the nicotinic acetylcholine receptor. Phys Biol, 3(2):147-159, 2006.

[19] P. Ball. Water as an active constituent in cell biology. Chemical Reviews, 108(1):74-108, 2008.

[20] L. Zhang, L. Wang, Y. Kao, W. Qiu, Y. Yang, O. Okobiah, and D. Zhong. Mapping hydration dynamics around a protein surface. Proc. Natl. Acad. Sci. U. S. A., 104(47):18461-18466, Nov 2007.

[21] S. K. Pal, J. Peon, and A. H. Zewail. Biological water at the protein surface: dynamical solvation probed directly with femtosecond resolution. Proc. Natl. Acad. Sci. U. S. A., 99(4):1763-1768, Feb 2002.

[22] B. Alberts, A. Johnson, J. Lewis, M. Raff, K. Roberts, and P. Walter. Molecular Biology of the Cell, Fourth Edition. Garland, 2002. 
[23] M. L. Zeidel, S. V. Ambudkar, B. L. Smith, and P. Agre. Reconstitution of functional water channels in liposomes containing purified red-cell CHIP28 protein. Biochemistry, 31:7436-7440, 1992.

[24] B. Corry and S. H. Chung. Mechanisms of valence selectivity in biological ion channels. Cell. Mol. Life Sci., 63(3):301-315, 2006.

[25] B. Roux and K. Schulten. Computational studies of membrane channels. Structure, 12(8):1343-1351, 2004.

[26] B. Roux. Ion conduction and selectivity in $\mathrm{K}^{+}$channels. Annu. Rev. Biophys. Biomol. Struct., 34:153-171, 2005.

[27] E. Gouaux and R. Mackinnon. Principles of selective ion transport in channels and pumps. Science, 310(5753):1461-1465, 2005.

[28] J. S. Hub and B. L. de Groot. Mechanism of selectivity in aquaporins and aquaglyceroporins. Proc. Natl. Acad. Sci. USA, 105(4):1198-1203, 2008.

[29] B. L. de Groot and H. Grubmüller. The dynamics and energetics of water permeation and proton exclusion in aquaporins. Curr. Opin. Struct. Biol., 15:176-183, 2005.

[30] P. Agre, M. Bonhivers, and M. J. Borgnia. The aquaporins, blueprints for cellular plumbing systems. J. Biol. Chem., 273:14659-14662, 1998.

[31] G. Hummer, J. C. Rasaiah, and J. P. Noworyta. Water conduction through the hydrophobic channel of a carbon nanotube. Nature, 414:188-190, 2001.

[32] O. Beckstein and M. S. Sansom. Liquid-vapor oscillations of water in hydrophobic nanopores. Proc. Natl. Acad. Sci. USA, 100:7063-7068, 2003.

[33] R. Allen, S. Melchionna, and J. P. Hansen. Intermittent permeation of cylindrical nanopores by water. Phys. Rev. Lett., 89(17):175502-, 2002.

[34] P. De Weer. A century of thinking about cell membranes. Annu. Rev. Physiol., 62:919-926, 2000.

[35] S. B. Hladky and D. A. Haydon. Discreteness of Conductance Change in Bimolecular Lipid Membranes in the Presence of Certain Antibiotics. Nature, 225:451-453, January 1970. 
[36] B. Sackmann and E. Neher, editors. Single Channel Recording. Plenum Press, 1995.

[37] R. Fettiplace and D. A. Haydon. Water permeability of lipid membranes. Physiol. Rev., 60(2):510-550, 1980.

[38] A. S. Verkman. Water permeability measurement in living cells and complex tissues. J Membr Biol, 173(2):73-87, Jan 2000.

[39] S. M. Saparov, Y. N. Antonenko, R. E. Koeppe II, and P. Pohl. Desformylgramicidin: a model channel with an extremely high water permeability. Biophys. J., 79:2526-2534, 2000.

[40] P. Pohl and S. M. Saparov. Solvent drag across gramicidin channels demonstrated by microelectrodes. Biophys. J., 78(5):2426-2434, May 2000.

[41] P. A. Rosenberg and A. Finkelstein. Water permeability of gramicidin A-treated lipid bilayer membranes. J. Gen. Physiol., 72:341-350, 1978.

[42] R. Holz and A. Finkelstein. The water and nonelectrolyte permeability induced in thin lipid membranes by the polyene antibiotics nystatin and amphotericin $b$. J Gen Physiol, 56(1):125-145, Jul 1970.

[43] P. H. Jensen and D. Keller. Membrane for filetering of water, 2006. Patent number WO 2006/122566.

[44] H. Bayley and P. S. Cremer. Stochastic sensors inspired by biology. Nature, 413(6852):226-230, Sep 2001.

[45] R. E. Koeppe and O. S. Anderson. Engineering the gramicidin channel. Annu Rev Biophys Biomol Struct, 25:231-258, 1996.

[46] P. Yin, C. J. Burns, P. D. J. Osman, and B. A. Cornell. A tethered bilayer sensor containing alamethicin channels and its detection of amiloride based inhibitors. Biosens. Bioelectron., 18(4):389-397, Apr 2003.

[47] M. R Banghart, M. Volgraf, and D. Trauner. Engineering light-gated ion channels. Biochemistry, 45(51):15129-15141, Dec 2006.

[48] H. Bayley. Designed membrane channels and pores. Curr. Opin. Biotechnol., 10(1):94-103, Feb 1999. 
[49] S. Futaki, Y. J. Zhang, T. Kiwada, I. Nakase, T. Yagami, S. Oiki, and Y. Sugiura. Gramicidin-based channel systems for the detection of protein-ligand interaction. Bioorg. med. chem., 12(6):1343-1350, 2004.

[50] B. A. Cornell, V. L. Braach-Maksvytis, L. G. King, P. D. Osman, B. Raguse, L. Wieczorek, and R. J. Pace. A biosensor that uses ion-channel switches. Nature, 387(6633):580-583, Jun 1997.

[51] A. Hirano, M. Wakabayashi, Y. Matsuno, and M. Sugawara. A single-channel sensor based on gramicidin controlled by molecular recognition at bilayer lipid membranes containing receptor. Biosens Bioelectron, 18(8):973-983, Aug 2003.

[52] V. Borisenko, Z. Zhang, and G. A. Woolley. Gramicidin derivatives as membranebased pH sensors. Biochim. Biophys. Acta, 1558(1):26-33, Jan 2002.

[53] M. R. Ghadiri, J. R. Granja, R. A. Milligan, D. E. McRee, and N. Khazanovich. Self-assembling organic nanotubes based on a cyclic peptide architecture. Nature, 366(6453):324-327, Nov 1993.

[54] M. R. Ghadiri, J. R. Granja, and L. K. Buehler. Artificial transmembrane ion channels from self-assembling peptide nanotubes. Nature, 369(6478):301-304, May 1994.

[55] K. Motesharei and M.R. Ghadiri. Diffusion-limited size-selective ion sensing based on sam-supported peptide nanotubes. J. Am. Chem. Soc., 119(46):1130611312, 1997.

[56] O. Braha, B. Walker, S. Cheley, J. J. Kasianowicz, L. Song, J. E. Gouaux, and H. Bayley. Designed protein pores as components for biosensors. Chem. Biol., 4(7):497-505, Jul 1997.

[57] D. G. Levitt. Kinetics of diffusion and convection in $3.2 \AA$ pores. Biophys. J., 13:186-206, 1973.

[58] B. Roux, T. W. Allen, S. Bernèche, and W. P. Im. Theoretical and computational models of biological ion channels. Quart. Rev. Biophys., 37:15-103, 2004.

[59] D. P. Tieleman, G. R. Smith, P. C. Biggin, and M. S. P. Sansom. Simulation approaches to ion channel structure-function relationships. Quart. Rev. Biophys., 34:473-561, 2001. 
[60] B. Roux. Theoretical and computational models of ion channels. Curr. Opin. Struct. Biol., 12(2):182-189, Apr 2002.

[61] B. L. de Groot, D. P. Tieleman, P. Pohl, and H. Grubmüller. Water permeation through gramicidin A: desformylation and the double helix; a molecular dynamics study. Biophys. J., 82:2934-2942, 2002.

[62] S. Berneche and B. Roux. Energetics of ion conduction through the $\mathrm{K}^{+}$channel. Nature, 414:73-77, 2001.

[63] B. L. de Groot and H. Grubmüller. Water permeation across biological membranes: Mechanism and dynamics of Aquaporin-1 and GlpF. Science, 294:2353$2357,2001$.

[64] S. M. Saparov, J. R. Pfeifer, L. Al-Momani, G. Portella, B. L. deGroot, U. Koert, and P. Pohl. Mobility of a one-dimensional confined file of water molecules as a function of file length. Phys. Rev. Lett., 96(14):148101, 2006.

[65] M.P. Allen and D.J. Tidesley. Computer simulations of liquids. Clarendon Press, Oxford, 1987.

[66] A. R. Leach. Molecular Modelling, Principles and applications. Prentice-Hall, 2001.

[67] D. Frenkel and B. Smit. Understanding Molecular Simulations. Academic Press, New York, 2002.

[68] W. F. Van Gunsteren and H. J. C. Berendsen. Computer simulation of molecular dynamics: Methodology, applications, and perspectives in chemistry. Angew. Chem. Int. Ed., 29:992-1023, 1990.

[69] W. F. van Gunsteren, D. Bakowies, R. Baron, I. Chandrasekhar, M. Christen, X. Daura, P. Gee, D. P. Geerke, A. Glättli, P. H. Hünenberger, M. A. Kastenholz, C. Oostenbrink, M. Schenk, D. Trzesniak, N. F. A. van der Vegt, and H. B. Yu. Biomolecular modeling: Goals, problems, perspectives. Angew Chem Int Ed Engl, 45(25):4064-4092, 2006.

[70] M. Karplus and J. A. McCammon. Molecular dynamics simulations of biomolecules. Nat Struct Biol, 9(9):646-652, Sep 2002.

[71] M. Born and R. Oppenheimer. Zur quantentheorie der molekeln. Ann. Phys., 84:457-484, 1927. 
[72] M. Born and K. Huang. Dynamical theory of crystal lattices. Oxford University Press, 1952.

[73] F. C. Brooks. Convergence of intermolecular force series. Phys. Rev., 86(1):9297, 1952.

[74] R. B. Gerber. On the order of accuracy of the born-oppenheimer approximation for molecuar collision states. Proc. Roy. Soc. London. Ser A - Math. and Phys. Sc., 309(1497):221-244, 1969.

[75] R.W. Hockney. The potential calculation and some applications. Methods Comput. Phys., 9:136-211, 1970.

[76] M. A. Cuendet and W. F. van Gunsteren. On the calculation of velocitydependent properties in molecular dynamics simulations using the leapfrog integration algorithm. The Journal of Chemical Physics, 127(18):184102, 2007.

[77] D. Marx and J. Hutter. Modern Methods and Algorithms of Quantum Chemistry, chapter Ab-initio molecular dynamics: Theory and implementation, pages 301449. NIC, FZ Jülich, 2000.

[78] G. A. Kaminski, R. A. Friesner, J. Tirado-Rives, and W. L. Jorgensen. Evaluation and reparametrization of the OPLS-AA force field for proteins via comparison with accurate quantum chemical calculations on peptides. J. Phys. Chem. $B, 105: 6474-6487,2001$.

[79] W. L. Jorgensen, D. S. Maxwell, and J. Tirado-Rives. Development and testing of the OPLS all-atom force field on conformational energetics and properties of organic liquids. J. Am. Chem. Soc., 118:11225-11236, 1996.

[80] W. L. Jorgensen, J. Chandrasekhar, J. D. Madura, R. W. Impey, and M. L. Klein. Comparison of simple potential functions for simulating liquid water. $J$. Chem. Phys., 79:926-935, 1983.

[81] W. F. van Gunsteren and H. J. C. Berendsen. Algorithms for macromolecular dynamics and constraint dynamics. Molecular Physics, 34:1311-1327, 1977.

[82] B. Hess, H. Bekker, H. J. C. Berendsen, and J. G. E. M. Fraaije. LINCS: A linear constraint solver for molecular simulations. J. Comp. Chem., 18:14631472, 1997. 
[83] S. Miyamoto and P. A. Kollman. SETTLE: An analytical version of the SHAKE and RATTLE algorithms for rigid water models. J. Comp. Chem., 13:952-962, 1992.

[84] M. Berkowitz and J. A. McCammon. Molecular dynamics with stochastic boundary conditions. Chemical Physics Letters, 90:215-217, July 1982.

[85] C. L. Brooks III and M. Karplus. Deformable stochastic boundaries in molecular dynamics. J. Chem. Phys., 79:6312-6325, 1983.

[86] W. Im, S. Bernèche, and B. Roux. Generalized solvent boundary potential for computer simulations. J. Chem. Phys., 114:2924-2937, February 2001.

[87] M. Saito. Molecular dynamics simulations of proteins in solution: Artifacts caused by the cutoff approximation. J. Chem. Phys., 101(5):4055-4061, 1994.

[88] M. Saito. Molecular dynamics simulations of proteins in solution with all degrees of freedom and long-range coulomb interactions. J. Phys. Chem., 99:1704317048, 1995.

[89] J.A. Barker and R.O. Watts. Monte carlo studies of the dielectric properties of water-like models. Molecular Physics, 26:789-792(4), 1973.

[90] I. Tironi, R. Sperb, P. E. Smith, and W. F. van Gunsteren. A generalized reaction field method for molecular dynamics simulations. J. Chem. Phys., 102:5451$5459,1995$.

[91] W.F. Van Gunsteren, S.R. Billeter, A.A. Eising, P.H. Hünenberger, P. Krüger, A.E. Mark, W.R.P. Scott, and I.G. Tironi. Biomolecular simulation: the GROMOS96 manual and user guide. Biomos b.v., Zürich, Groningen, 1996.

[92] A. Warshel, P. K Sharma, M. Kato, and W. W. Parson. Modeling electrostatic effects in proteins. Biochim. Biophys. Acta, 1764(11):1647-1676, Nov 2006.

[93] T. Darden, D. York, and L. Pedersen. Particle mesh Ewald: an N·log(N) method for Ewald sums in large systems. J. Chem. Phys., 98:10089-10092, 1993.

[94] R. W. Hockney and J. W. Eastwood. Computer simulation using particles. Taylor \& Francis, Inc., Bristol, PA, USA, 1988.

[95] H. J. C. Berendsen, J. P. M. Postma, A. DiNola, and J. R. Haak. Molecular dynamics with coupling to an external bath. J. Chem. Phys., 81:3684-3690, 1984. 
[96] F. Zhu, E. Tajkhorshid, and K. Schulten. Theory and simulation of water permeation in aquaporin-1. Biophys. J., 86:50-57, 2004.

[97] F. Zhu, E. Tajkhorshid, and K. Schulten. Collective diffusion model for water permeation through microscopic channels. Phys. Rev. Lett., 93(22):224501, Nov 2004 .

[98] G. Arya, H. C. Chang, and E. J. Maginn. A critical comparison of equilibrium, non-equilibrium and boundary-driven molecular dynamics techniques for studying transport in microporous materials. J. Chem. Phys., 115(17):8112-8124, 2001.

[99] A. Berezhkovskii and G. Hummer. Single-file transport of water molecules through a carbon nanotube. Phys. Rev. Lett., 89(6):064503, Aug 2002.

[100] P. Hänggi, P. Talkner, and M. Borkovec. Reaction-rate theory: fifty years after kramers. Rev. Mod. Phys., 62(2):251-341, Apr 1990.

[101] J. A. Hernandez and J. Fischbarg. Kinetic analysis of water transport though a single-file pore. J. Gen. Physiol., 99(4):645-662, 1992.

[102] Donald A. Mcquarrie. Statistical Mechanics. University Science Books, June 2000 .

[103] P M Chaikin and T C Lupensky. Principles of condensed matter physics. Cambridge University Press, Cambridge, 2000.

[104] J. G. Kirkwood. Statistical mechanics of fluid mixtures. J. Chem. Phys., 3(5):300-313, 1935.

[105] R. W. Zwanzig. Time-correlation functions and transport coefficients in statistical mechanics. Annu. Rev. Phys. Chem., 16:67-102, 1965.

[106] R. W. Zwanzig. High-temperature equation of state by a perturbation method. 1. Nonpolar gases. J. Chem. Phys., 22:1420-1426, 1954.

[107] T. Straatsma, , and J. A. McCammon. Computational alchemy. Ann. Rev. Phys. Chem., 43:407-435, 1992.

[108] H. Meirovitch. Recent developments in methodologies for calculating the entropy and free energy of biological systems by computer simulation. Curr. Opin. Struct. Biol., 17(2):181-186, Apr 2007. 
[109] B. Widom. Some topics in theory of fluids. J. Chem. Phys., 39:2808, 1963.

[110] E. A. Carter and. G. Ciccotti, J. T. Hynes, and R. Kapral. Constrained reaction coordinate dynamics for the simulation of rare events. Chem. Phys. Lett., 156:472-477, 1989 .

[111] I.R. McDonald and J.C. Rasaiaha. Monte carlo simulation of the average force between two ions in a stockmayer solvent. Chem. Phys. Lett., 34:382-386, 1975.

[112] G. M. Torrie and J. P. Valleau. Monte Carlo free energy estimates using nonBoltzmann sampling: Application to the sub-critical Lennard-Jones fluid. Chem. Phys. Lett., 28:578-581, 1974.

[113] C. Jarzynski. Equilibrium free-energy differences from nonequilibrium measurements: A master-equation approach. Phys. Rev. E, 56:5018-5035, 1997.

[114] G. E. Crooks. Path-ensemble averages in systems driven far from equilibrium. Phys. Rev. E, 61(3):2361-2366, Mar 2000.

[115] J. Gore, F. Ritort, and C. Bustamante. Bias and error in estimates of equilibrium free-energy differences from nonequilibrium measurements. Proc. Natl. Acad. Sci. U. S. A., 100(22):12564-12569, Oct 2003.

[116] D. J. Evans and D. J. Searles. Equilibrium microstates which generate second law violating steady states. Phys. Rev. E, 50(2):1645-1648, Aug 1994.

[117] D. J. Evans and D. J. Searles. Causality, response theory, and the second law of thermodynamics. Phys. Rev. E, 53(6):5808-5815, Jun 1996.

[118] H. Grubmüller, B. Heymann, and P. Tavan. Ligand binding: Molecular mechanics calculation of the streptavidin-biotin rupture force. Science, 271:997-999, 1996.

[119] G. Hummer. Fast-growth thermodynamic integration: Error and efficiency analysis. J Chem Phys, 114:7330-7337, 2001.

[120] D. A. Hendrix and C. Jarzynski. A "fast growth" method of computing free energy differences. J. Chem. Phys., 114(14):5974-5981, 2001.

[121] B. Roux. The calculation of the potential of mean force using computer simulations. Computer Physics Communications, 91:275-282, September 1995. 
[122] S. Kumar, D. Bouzida, R. H. Swendsen, P. A. Kollman, and J. M. Rosenberg. The weighted histogram analysis method for free-energy calculations on biomolecules. I. the method. J. Comp. Chem., 13:1011-1021, 1992.

[123] A. Finkelstein and P.A. Rosenberg. Single-file transport: Implications for ion and water movement through gramicidin a channels. Membrane Transport Processe, 3:77-88, 1979.

[124] S. W. Chiu, S. Subramaniam, and E. Jakobsson. Simulation study of the gramicidin/lipid bilayer system in excess water and lipid. II. Rates and mechanism of water transport. Biophys. J., 76:1939-1950, 1999.

[125] J. A. Dani and D. G. Levitt. Water transport and ion-water interaction in the gramicidin channel. Biophys. J., 35:501-508, 1981.

[126] O. S. Andersen, H. J. Apell, E. Bamberg, D. D. Busath, R. E. Koeppe, F. J. Sigworth, G. Szabo, D. W. Urry, and A. Woolley. Gramicidin channel controversythe structure in a lipid environment. Nat Struct Biol, 6(7):609; discussion 611609; discussion 612, Jul 1999.

[127] T. W. Allen, O. S. Andersen, and B. Roux. Structure of gramicidin a in a lipid bilayer environment determined using molecular dynamics simulations and solid-state nmr data. J. Am. Chem. Soc., 125(32):9868-9877, Aug 2003.

[128] F. Kovacs, J. Quine, and T. A. Cross. Validation of the single-stranded channel conformation of gramicidin a by solid-state nmr. Proc Natl Acad Sci USA, 96(14):7910-7915, Jul 1999.

[129] X. Xie, L. Al-Momani, P. Reiss, C. Griesinger, and U. Koert. An asymmetric ion channel derived from gramicidin a. synthesis, function and $\mathrm{nmr}$ structure. FEBS J., 272(4):975-986, Feb 2005.

[130] H. D. Arndt, D. Bockelmann, A. Knoll, S. Lamberth, C. Griesinger, and U. Koert. Cation control in functional helical programming: structures of a d,l-peptide ion channel. Angew. Chem. Int. Ed., 41(21):4062-4065, Nov 2002.

[131] R. R. Ketchem, B. Roux, and T. A. Cross. High-resolution polypeptide structure in a lamellar phase lipid environment from solid state NMR derived orientational constraints. Structure, 5:1655-1669, 1997. 
[132] J. D. Faraldo-Gómez, G. R. Smith, and M. S. Sansom. Setting up and optimization of membrane protein simulations. Europ. Biophys. J., 31:217-227, 2002.

[133] O. Berger, O. Edholm, and F. Jähnig. Molecular dynamics simulations of a fluid bilayer of dipalmitoylphosphatidylcholine at full hydration, constant pressure, and constant temperature. Biophys. J., 72:2002-2013, 1997.

[134] E. Lindahl, B. Hess, and D. Van der Spoel. GROMACS 3.0: a package for molecular simulation and trajectory analysis. J. Mol. Model., 7:306-317, 2001.

[135] D. Van der Spoel, E. Lindahl, B. Hess, G. Groenhof, A. E. Mark, and H. J. C. Berendsen. GROMACS: Fast, flexible and free. J. Comput. Chem., 26:1701$1718,2005$.

[136] U. Essmann, L. Perera, M. L. Berkowitz, T. Darden, H. Lee, and L. G. Pedersen. A smooth particle mesh ewald potential. J. Chem. Phys., 103:8577-8592, 1995.

[137] Oliver S. Smart, Joseph G. Neduvelil, Xiaonan Wang, B. A. Wallace, and Mark S. P. Sansom. Hole: A program for the analysis of the pore dimensions of ion channel structural models. J. Mol. Graphics, 14:354-360, 1996.

[138] M. R. R. dePlanque, J. A. W. Kruijtzer, R. M. J. Liskamp, D. Marsh, D. V. Greathouse, R. E. Koeppe, B. deKruijff, and J. A. Killian. Different membrane anchoring positions of tryptophan and lysine in synthetic transmembrane alphahelical peptides. J. Biol. Chem., 274(30):20839-20846, 1999.

[139] H. Tournois, P. Gieles, R. Demel, J. de Gier, and B. de Kruijff. Interfacial properties of gramicidin and gramicidin-lipid mixtures measured with static and dynamic monolayer techniques. Biophys J, 55(3):557-569, Mar 1989.

[140] S. W. Chiu, S. Subramaniam, and E. Jakobsson. Simulation study of the gramicidin/lipid bilayer system in excess water and lipid. I. Structure of the molecular complex. Biophys. J., 76:1929-1938, 1999.

[141] T. M. Weiss, P. C A van der Wel, J. A. Killian, R. E. Koeppe, and H. W. Huang. Hydrophobic mismatch between helices and lipid bilayers. Biophys. J., 84(1):379-385, Jan 2003.

[142] O. S. Andersen and R. E. Koeppe. Bilayer thickness and membrane protein function: an energetic perspective. Annu. Rev. Biophys. Biomol. Struct., 36:107130, 2007. 
[143] A. E. Daily, D. V. Greathouse, P. C. A van der Wel, and R. E. Koeppe. Helical distortion in tryptophan- and lysine-anchored membrane-spanning alpha-helices as a function of hydrophobic mismatch: a solid-state deuterium $\mathrm{nmr}$ investigation using the geometric analysis of labeled alanines method. Biophys. J., 94(2):480491, Jan 2008.

[144] P. Pohl and S. M. Saparov. Solvent drag across gramicidin channels demonstrated by microelectrodes. Biophys. J., 78:2426-2434, 2000.

[145] A. L. Lomize, V. Iu Orekhov, and A. S. Arsen'ev. Refinement of the spatial structure of the gramicidin a ion channel. Bioorg Khim, 18(2):182-200, Feb 1992.

[146] L. E. Townsley, W. A. Tucker, S. Sham, and J. F. Hinton. Structures of gramicidins a, b, and c incorporated into sodium dodecyl sulfate micelles. Biochemistry, 40(39):11676-11686, Oct 2001.

[147] A. R. Jude, D. V. Greathouse, R. E. Koeppe, L. L. Providence, and O. S. Andersen. Modulation of gramicidin channel structure and function by the aliphatic "spacer" residues 10, 12, and 14 between the tryptophans. Biochemistry, 38(3):1030-1039, 1999.

[148] R. A. F. Reithmeier. Charecterization and modeling of membrane-proteins using sequence-analysis. Curr. Opin. Struct. Biol., 5(4):491-500, 1995.

[149] H. J. C. Berendsen, J. P. M. Postma, W. F. van Gunsteren, and J. Hermans. Interaction models for water in relation to protein hydration. In B. Pullman, editor, Intermolecular Forces, pages 331-342. D. Reidel Publishing Company, Dordrecht, 1981.

[150] A. Kalra, S. Garde, and G. Hummer. Osmotic water transport through carbon nanotube membranes. Proc. Natl. Acad. Sci. USA, 100:10175-10180, 2003.

[151] P. Pohl. Combined transport of water and ions through membrane channels. Biol. Chem., 385(10):921-926, Oct 2004.

[152] J. R. Pfeifer, P. Reiss, and U. Koert. Crown ether-gramicidin hybrid ion channels: Dehydration-assisted ion selectivity. Angew. Chem. Ind. Ed., 45(3):501-504, 2006. 
[153] H. C. Longuet-Higgins and G. Austin. The kinetics of osmotic transport through pores of molecular dimensions. Biophys J, 6(2):217-224, Mar 1966.

[154] R.J. Mashl, S. Joseph, N.R. Aluru, and E. Jakobsson. Anomalously immobilized water: A new water phase induced by confinement in nanotubes. Nano Letters, 3(5):589-592, 2003.

[155] O. Beckstein and M. S. P. Sansom. The influence of geometry, surface character, and flexibility on the permeation of ions and water through biological pores. Phys Biol, 1(1-2):42-52, Jun 2004.

[156] O. Beckstein, K. Tai, and M. S. P. Sansom. Not ions alone: barriers to ion permeation in nanopores and channels. J Am Chem Soc, 126(45):14694-14695, Nov 2004.

[157] T. Chou. How fast do fluids squeeze through microscopic single-file pores? Phys. Rev. Lett., 80(1):85-88, Jan 1998.

[158] J. R. Pappenheimer. Passage of molecules through capillary walls. Physiol. Rev., 33:387-432, 1953.

[159] C. V. Paganelli and A. K. Solomon. The rate of exchange of tritiated water across the human red cell membrane. J. Gen. Physiol., 41:259-277, 1957.

[160] D. G. Levitt. Dynamics of a single-file pore: non-Fickian behavior. Phys. Rev. A, 8(6):3050-3054, 1973.

[161] P. M. Richards. Theory of one-dimensional hopping conductivity and diffusion. Phys. Rev. B, 16(4):1393-1409, 1977.

[162] S. Alexander and P. Pincus. Diffusion of labeled particles on one-dimensional chains. Phys. Rev. B, 18(4):2011-2012, 1978.

[163] Peter A. Fedders. Two-point correlation functions for a distinguishable particle hopping on a uniform one-dimensional chain. Phys. Rev. B, 17(1):40-46, 1978.

[164] T.W. Allen, S. Kuyucak, and S. H. Chung. The effect of hydrophobic and hydrophilic channel walls on the structure and diffusion of water and ions. $J$. Chem. Phys., 111:7985-7999, November 1999.

[165] M. Tarek, B. Maigret, and C. Chipot. Molecular dynamics investigation of an oriented cyclic peptide nanotube in dmpc bilayers. Biophys. J., 85(4):2287-2298, Oct 2003. 
[166] C. P. Bean. The physics of porous membranes-neutral pores. Membranes, 1:1-54, 1972.

[167] S. M. Saparov and P. Pohl. Beyond the diffusion limit: Water flow through the empty bacterial potassium channel. Proc. Natl. Acad. Sci. U. S. A., 101(14):4805-4809, Apr 2004.

[168] Hahn, Kärger, and Kukla. Single-file diffusion observation. Phys Rev Lett, 76(15):2762-2765, Apr 1996.

[169] B. A. Wallace. Common structural features in gramicidin and other ion channels. Bioessays, 22(3):227-234, Mar 2000.

[170] D. A. Doyle, J. M. Cabral, R. A. Pfuetzner, A. L. Kuo, J. M. Gulbis, S. L. Cohen, B. T. Chait, and R. MacKinnon. The structure of the potassium channel: Molecular basis of K+ conduction and selectivity. Science, 280:69-77, 1998.

[171] Sergei Yu Noskov and Benoit Roux. Ion selectivity in potassium channels. Biophys Chem, 124(3):279-291, Dec 2006.

[172] D. L. Bostick and C. L. Brooks. Selectivity in K+ channels is due to topological control of the permeant ion's coordinated state. Proc. Natl. Acad. Sci. U. S. A., 104(22):9260-9265, May 2007.

[173] Y. Zhou, J. H. Morais-Cabral, A. Kaufman, and R. MacKinnon. Chemistry of ion coordination and hydration revealed by a $\mathrm{k}+$ channel-fab complex at $2.0 \mathrm{a}$ resolution. Nature, 414(6859):43-48, Nov 2001.

[174] O. S. Andersen. Gramicidin channels. Annu. Rev. Physiol., 46:531-548, 1984.

[175] O. S. Andersen and R. E. Koeppe. Molecular determinants of channel function. Physiol Rev, 72(4 Suppl):S89-158, Oct 1992.

[176] Y. N. Antonenko, T. I. Rokitskaya, E. A. Kotova, G. O. Reznik, T. Sano, and C. R. Cantor. Effect of streptavidins with varying biotin binding affinities on the properties of biotinylated gramicidin channels. Biochemistry, 43(15):4575-4582, Apr 2004.

[177] R. Capone, S. Blake, M. R. Restrepo, J. Yang, and M. Mayer. Designing nanosensors based on charged derivatives of gramicidin a. J. Am. Chem. Soc., 129(31):9737-9745, Aug 2007. 
[178] C. Peter and G. Hummer. Ion transport through membrane-spanning nanopores studied by molecular dynamics simulations and continuum electrostatics calculations. Biophys J, 89(4):2222-2234, Oct 2005.

[179] S. H. Chung and B. Corry. Three computational methods for studying permeation, selectivity and dynamics in biological ion channels. Soft Matter, 1:417-457, 2005.

[180] S. Bernèche and B. Roux. A microscopic view of ion conuction through the $\mathrm{K}^{+}$ channel. Proc. Natl. Acad. Sci. USA, 100:8644-8648, 2003.

[181] T. W. Allen, O. S. Andersen, and B. Roux. On the importance of atomic fluctuations, protein flexibility, and solvent in ion permeation. J. Gen. Physiol., 124(6):679-690, Dec 2004.

[182] T.Bastug, A. Gray-Weale, S. M. Patra, and S. Kuyucak. Role of protein flexibility in ion permeation: a case study in gramicidin a. Biophys. J., 90(7):2285-2296, Apr 2006.

[183] P. S. Crozier, R. L. Rowley, N. B. Holladay, D. Henderson, and D. D. Busath. Molecular dynamics simulation of continuous current flow through a model biological membrane channel. Phys Rev Lett, 86(11):2467-2470, Mar 2001.

[184] J. Aqvist. Ion-water interaction potentials derived from free energy perturbation simulations. J. Phys. Chem., 94:8021:8024, 1990.

[185] Jayaraman Chandrasekhar, David C. Spellmeyer, and William L. Jorgensen. Energy component analysis for dilute aqueous solutions of lithium(1+), sodium(1+), fluoride(1-), and chloride(1-) ions. Journal of the American Chemical Society, 106(4):903-910, 1984.

[186] D. L. Beveridge and F. M. DiCapua. Free energy via molecular simulation: applications to chemical and biomolecular systems. Annu Rev Biophys Biophys Chem, 18:431-492, 1989.

[187] P. A. Kollman. Free energy calculations: applications to chemical and biochemical phenomena. Chem. Rev., 93:2395-2417, 1993.

[188] Christine Peter, Chris Oostenbrink, Arthur van Dorp, and Wilfred F van Gunsteren. Estimating entropies from molecular dynamics simulations. $J$ Chem Phys, 120(6):2652-2661, Feb 2004. 
[189] S. Wan, R. H Stote, and M. Karplus. Calculation of the aqueous solvation energy and entropy, as well as free energy, of simple polar solutes. J. Chem. Phys., 121(19):9539-9548, Nov 2004.

[190] Jurgen Schlitter. Estimation of absolute and relative entropies of macromolecules using the covariance matrix. Chem. Phys. Lett., 215:617-621, 1993.

[191] I. Andricioaei and M. Karplus. On the calculation of entropy from covariance matrices of the atomic fluctuations. J. Chem. Phys., 115:6289-6292, 2001.

[192] T. W. Allen, O. S. Andersen, and B. Roux. Ion permeation through a narrow channel: using gramicidin to ascertain all-atom molecular dynamics potential of mean force methodology and biomolecular force fields. Biophys J, 90(10):34473468, May 2006.

[193] J. Aqvist and A. Warshel. Energetics of ion permeation through membrane channels. solvation of na+ by gramicidin a. Biophys J, 56(1):171-182, Jul 1989.

[194] B. Roux. Statistical mechanical equilibrium theory of selective ion channels. Biophys J, 77(1):139-153, Jul 1999.

[195] A. Parsegian. Energy of an ion crossing a low dielectric membrane: solutions to four relevant electrostatic problems. Nature, 221(5183):844-846, Mar 1969.

[196] D. G. Levitt. Electrostatic calculations for an ion channel. i. energy and potential profiles and interactions between ions. Biophys J, 22(2):209-219, May 1978.

[197] P. C. Jordan. Electrostatic modeling of ion pores. energy barriers and electric field profiles. Biophys J, 39(2):157-164, Aug 1982.

[198] S. Teber. Translocation energy of ions in nano-channels of cell membranes. $J$. Stat. Mech., 7:1-17, July 2005.

[199] T. W. Allen, O. S. Andersen, and B. Roux. Energetics of ion conduction through the gramicidin channel. Proc. Natl. Acad. Sci. USA, 101:117-122, 2003.

[200] P. C. Jordan. Total electrostatic potential in a gramicidin channel. J. Membr. Biol., 78:91-102, 1984.

[201] P. C. Jordan. Ion permeation and chemical kinetics. J Gen Physiol, 114(4):601603, Oct 1999. 
[202] D. W. Urry, S. Alonso-Romanowski, C. M. Venkatachalam, R. J. Bradley, and R. D. Harris. Temperature dependence of single channel currents and the peptide libration mechanism for ion transport through the gramicidin a transmembrane channel. J Membr Biol, 81(3):205-217, 1984.

[203] R. M. Lynden-Bell and J. C. Rasaiah. From hydrophobic to hydrophilic behaviour: A simulation study of solvation entropy and free energy of simple solutes. J. Chem. Phys., 107(6):1981-1991, 1997.

[204] M. B. Partenskii, M. Cai, and P. C. Jordan. A dipolar chain model for the electrostatics of transmembrane ion channels. Chemical Physics, 153:125-131, 1991.

[205] M. B. Partenskii and P. C. Jordan. Nonlinear dielectric behavior of water in transmembrane ion channels: ion energy barriers and the channel dielectric constant. Journal of Physical Chemistry, 96(9):3906-3910, 1992.

[206] B. Roux and R. MacKinnon. The cavity and pore helices in the KcsA K ${ }^{+}$channel: electrostatic stabilization of monovalent cations. Science, 285(5424):100-102, Jul 1999.

[207] I. H. Shrivastava and M. S. P. Sansom. Simulations of ion permeation through a potassium channel: Molecular dynamics of KcsA in a phospholipid bilayer. Biophys. J., 78:557-570, 2000.

[208] J. Åqvist and V. Luzhkov. Ion permeation mechanism of the potassium channel. Nature, 404:881-884, 2000.

[209] G. Portella, P. Pohl, and B. L de Groot. Invariance of single-file water mobility in gramicidin-like peptidic pores as function of pore length. Biophys. J., 92(11):3930-3937, Jun 2007.

[210] D. W. Scott. On optimal and data-based histograms. Biometrika, 66:605-610, 1979.

[211] Ö. Eğecioğlu and A. Srinivasan. Efficient nonparametric density estimation on the sphere with applications in fluid mechanis. SIAM J. Sci. Comput., 22:152176,2000 . 


\section{LEBENSLAUF VON Guillem Portella Carbó}

\section{PERSÖNLICHE DATEN}

Name

Adresse

Geburtstag, -ort

Nationalität

Ausbildung

1995-1999

1999-2004

2006

2004-2008
Guillem Portella Carbó

Rudolf Diesel Strasse 1, 37075 Göttingen

1. Marz 1981 in Girona (Catalunya, Spanien)

Spanisch

High School - I.E.S. Jaume Vicens Vives, Girona.

Abitur (Selectivitat)

Universitat de Girona, Girona

Abschluss in Chemie (Llicenciat en Química)

Universitat de Barcelona, Barcelona

Graduiertenkolleg in computergestützter und theoretischer Chemie (Cursos de doctorado)

Max Planck Inst. für Biophysikalische Chemie, Göttingen. Dissertation mit dem Titel: "Determinants of water and ion permeation through nanopores studied by Molecular Dynamics simulations"

Betreuer: Dr. Bert L. de Groot 